\title{
Stone Exposure Test Wall
}

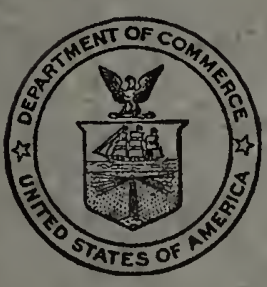

United States Department of Commerce

National Bureau of Standards

Building Materials and Structures Report 125 


\section{BUILDING MATERIALS AND STRUCTURES REPORTS}

On request, the Superintendent of Documents, U. S. Government Printing Office, Washington $25, \mathrm{D}$. C., will place your name on a special mailing list to receive notices of new reports in this series as soon as they are issued. There will be no charge for receiving such notices.

An alternative method is to deposit with the Superintendent of Documents the sum of $\$ 5$, with the request that the reports be sent to you as soon as issued, and that the cost thereof be charged against your deposit. This will provide for the mailing of the publications withrout delay. You will be notified when the amount of your deposit has become exhausted.

If 100 copies or more of any report are ordered at one time, a discount of 25 percent is allowed. Send all orders and remittances to the Superintendent of Documents, U. S. Government Printino Office, Washington $25, D$. C.

The following publications in this series are available by purchase from the Superintendent of Documents at the prices indicated:

BMS1

BMS2

BMS3

BMS4

BMS5

BMS6

BMS7

BMS8

BMS9

Research on Building Materials and Structures for Use in Low-Cost Housing.

Methods of Determining the Structural Properties of Low-Cost House Constructions_........ $10 k$

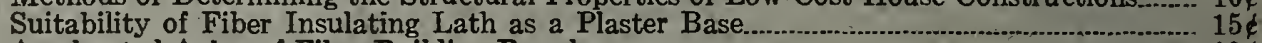

Accelerated Aging of Fiber Building Boards

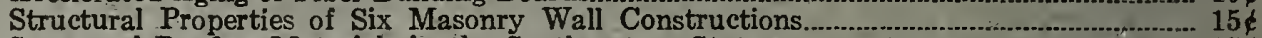

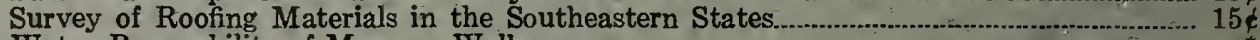

Water Permeability of Masonry Walls..

Methods of Investigation of Surface Treatment for Corrosion Protection of Steel.-.-.-..... 15

Structural Properties of the Insulated Steel Construction Co.'s "Frameless-Steel"' Constructions for Walls, Partitions, Floors, and Roofs.

BMS10 Structural Properties of One of the "Keystone Beam Steel Floor" Constructions Sponsored by the $\mathrm{H}$. $\mathrm{H}$. Robertson $\mathrm{Co}$.

BMS11 Structural Properties of the Curren Fabrihome Corporation's "Fabrihome" Constructions

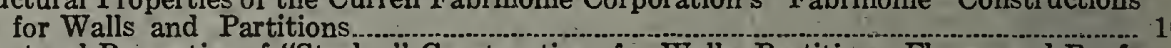

BMS12 Structural Properties of "Steelox" Constructions for Walls, Partitions, Floors, and Roofs Sponsored by Steel Buildings, Inc.

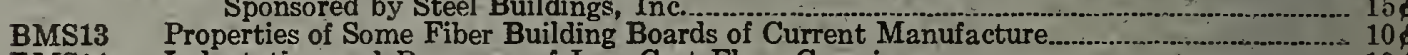

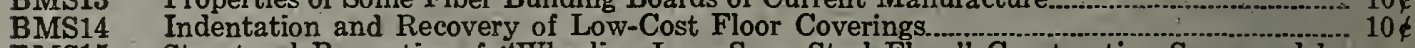

BMS15 - Structural Properties of "Wheeling Long-Span Steel Floor" Construction Sponsored by the Wheeling Corrugating Co.

BMS16 Structural Properties of a "Tilecrete" Floor Construction Sponsored by Tilecrete Floors, Inc.

BMS17 Sound Insulation of Wall and Floor Constructions

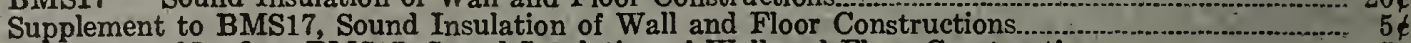

Supplement No. 2 to BMS17, Sound Insulation of Wall and Floor Construetions.............................. 15

BMS18 Structural Properties of "Pre-fab" Constructions for Walls, Partitions, and Floors Sponsored by the Harnischfeger Corporation.

BMS19 Preparation and Revision of Building Codes...
BMS20 Structural Properties of "Twachtman" Constructions for Walls and Floors Sponsored by

Connecticut Pre-Cast Buildings Corporation.
Structural Properties of a Concrete-Block Cavity-Wall Construction Sponsored by the
National Concrete Masonry Association......................

BMS21 National Concrete Masonry Association...

BMS22 Dunn Manufacturing Co.

BMS23 Structural Properties of a Brick Cavity-Wall Construction Sponsored by the Brick Manufacturers Association of New York, Inc.

BMS24 Structural Properties of a Reinforced-Brick Wall Construction and a Brick-Tile CavityWall Construction Sponsored by the Structural Clay Products Institute...-........ 15

BMS25 Structural Properties of Conventional Wood-Frame Constructions for Walls, Partitions, Floors, and Roofs.

BMS26 Structural Properties of "Nelson Pre-Cast Concrete Foundation" Wall Construction Spon- sored by the Nelson Cement Stone Co., Inc. _.

BMS27 Structural Properties of "Bender Steel Home" Wall Construction Sponsored by the Bender Body Co.

BMS28

BMS29

BMS30

Backflow Prevention in Over-Rim Water Supplies.

Survey of Roofing Materials in the Northeastern States

Structural Properties of a Wood-Frame Wall Construction Sponsored by the Douglas

BMS31 Structural Propertiso of "Insulite" Wall and "Insulite" Partition Constructions Sponsored

*out of print.

tSuperseded by BMS116.

[List continued on cover page III] 


\title{
Stone Exposure Test Wall
}

\author{
Daniel W. Kessler and R. E. Anderson
}

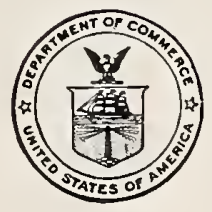

\section{Building Materials and Structures Report 125}

Issued September 20, 1951 



\section{Foreword}

The exposure test wall was built at the National Bureau of Standards in connection with a long-time project to study the action of various weathering agents affecting stone masonry. The project is a cooperative investigation between the National Bureau of Standards and Committee C-18 on Natural Building Stones of the American Society for Testing Materials.

This report sets forth problems on weathering, discoloration, and certain construction features. The four tables will serve as a permanent record of the individual samples in the wall. It is proposed to make detailed studies on the various materials as weathering becomes apparent and to issue additional reports when the data are available.

E. U. Condon, Director. 


\section{Contents}

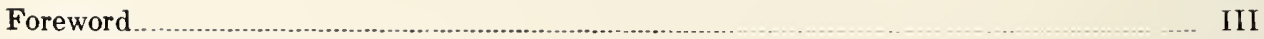

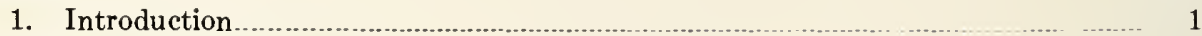

2. Construction features ........................... 1

3. Orientation and exposure conditions $\ldots$

4. Arrangement of stones in wall

5. Problems under study ............ 3

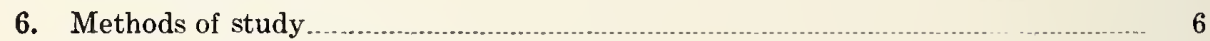

6.1. Weathering effects

6.2. Color changes........................... 6

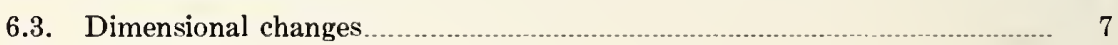

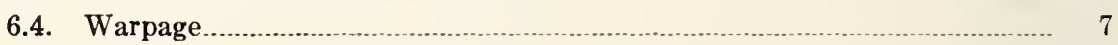

7. Maintenance of records

8. Catalog of stones in wall

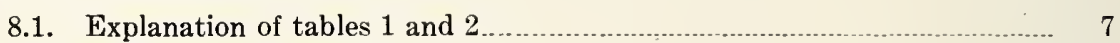

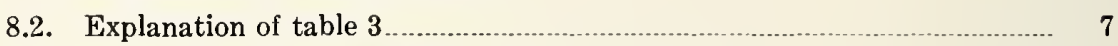

8.3. Stones in back and ends of wall 


\title{
Stone Exposure Test Wall
}

\author{
Daniel W. Kessler and R. E. Anderson
}

\begin{abstract}
A test wall was built at the Nationol Bureau of Standards during the months of September, October, and November 1948 in a plot set aside on the Bureau grounds for weathering tests of structural materials. The wall coritains 2,059 samples of stone in the front, or south face, and 293 in the back and ends; of these, 2.032 were domestic stones supplied by 47 States, and 320 were foreign samples supplied by 16 countries.

Stones in the east half of the south face are set in lime mortar with the exception of those in the base. All other stones in the wall are set in a portland cement mortar with a small addition of whiting. The core of the wall is of concrete, reinforced with steel bars.

The wall contairis 30 distinct types of stones, some of which are not commonly used for building purposes. There are many varieties of the usual types, such as marble, limestone, sandstone, and granite. The variations in composition or physical properties, such as strength, porosity, and density, will be of value in studying the relation between such properties and weather resistance.

The main problem is to determine the nature and relative severity of the various agents that cause deterioration and how they affect different stones. Certain structural features of the wall, such as length changes, warpage, back waterproofing, and grade waterproofing, are being studied in relation to weathering and discolorations on the stonework.

Each block is identified by a number determined by its position in the wall. This eliminates the necessity of frequent relabeling. The various samples are described under the wall numbers in tables that give specific information on location of stone deposits, classification, color, texture, and geological age, when known.
\end{abstract}

\section{Introduction}

During the latter part of 1942 , the National Museum in Washington, D. C. found it necessary to seek more working space, and it was decided to discard a large collection of building stones that had been assembled over a period of 60 years. A committee was appointed to consider whether or not any worthwhile use could be made of the collection. It was decided that a study of actual weathering on such a great variety of stones would afford valuable information. A plan was worked out for building a test wall at the National Bureau of Standards as a cooperative study between this Bureau and Committee C-18 of the American Society for Testing Materials.

The collection was stored at the National Bureau of Standards until the end of World War II, when the work of sorting, identifying, and trimming was started.

The sizes, shapes, and finishes were such that a great deal of time was required to recut many of the blocks before a logical design for the wall could be worked out. The original collection was supplemented with several larger blocks of granite, marble, limestone, sandstone, and greenstone supplied from present-day quarries. Test specimens ${ }^{1}$ were cored or sawed from all of the new and many of the old blocks before the wall was built. These specimens were taken from the back portions of blocks, at least three inches from the faces to be exposed to the weather. A considerable number of the original samples had rough faces that had to be removed. The cuttings were labeled and retained for future use in determining the nature of weathering processes.

The test wall was built at the National Bureau of Standards during September, October, and November of 1948 in a plot set aside by the Bureau for weathering tests of structural materials. Since the weathering of stone is usually a slow process, it is evident that the test should extend over a period of many years. The ultimate value will depend upon how future generations follow through in analyzing the results. The findings are expected to lead to the formulation of improved durability tests and specifications for masonry, which should result in more satisfactory buildings from the standpoint of economy and artistic appearance. Some useful information on discolorations and weathering of certain stones has already been obtained.

Aside from the objectives stated, this wall will serve to preserve a valuable collection of building stones, and the catalog of samples should be useful as a reference for builders in determining what kinds of stones are available in many localities.

\section{Construction Features}

The wall is approximately $37 \mathrm{ft} .9 \mathrm{in}$. long, $12 \mathrm{ft}$. $10 \mathrm{in}$. high, $2 \mathrm{ft}$. thick at the bottom and $1 \mathrm{ft}$. at

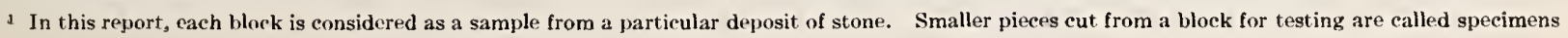




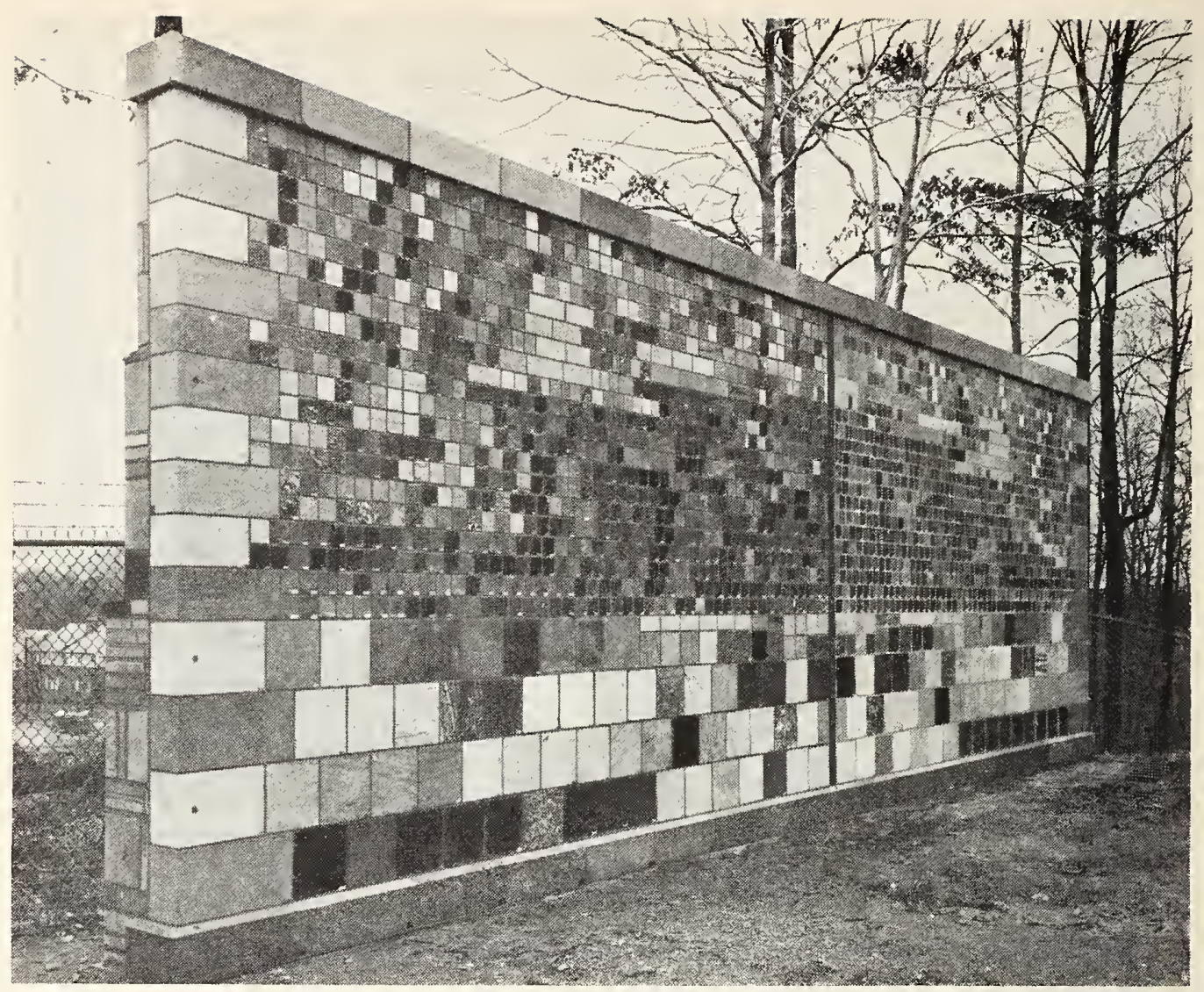

Figure 1. South face and west end of exposure test wall.

Photographed 1 month after erection.

the top. The south face, shown in figure 1, includes a granite base, limestone coping, marble quoins on the west end, and sandstone quoins on the east end. The photograph of the wall was taken 1 month after its completion, and shows the south face and west end. The stone facing varies in thickness, most of the blocks below course 5 being 5 in. or more thick and above course 4 , 4 in. or less. The divider strip extends 8 in. into the wall. A record of the dimensions of all blocks in the south face is maintained in the files. The wall is built on a concrete foundation which extends 30 in. below grade. Grade waterproofings under the base course are (1) two layers of $1 / 1 \mathrm{in}$. Virginia slate, laid with broken joints in cement mortar under base blocks $1 \mathrm{~B}, 2 \mathrm{~B}, 3 \mathrm{~B}, 15 \mathrm{~B}, 16 \mathrm{~B}$, and $17 \mathrm{~B} ;(2)$ one layer of $18-0 \%$. copper under base blocks $7 \mathrm{~B}, 8 \mathrm{~B}, 9 \mathrm{~B}, 10 \mathrm{~B}$, and $11 \mathrm{~B}$. Both slate and copper extend 18 in. into the wall and the intervening sections of the base course have no grade waterproofing. The stonework was built up in sections about $2 \mathrm{ft}$. high, after which the concrete backing was poured to this level.

The back of the stone facing above the copper grade waterproofing was treated with a brush coat of asphalt emulsion, which was allowed to dry before the concrete was poured. The concrete back- ing was reinforced as shown in figure 2 , and perforated 18-gage galvanized iron strips were used at frequent intervals to tie the stone facing to the concrete backing. The locations of these strips are also shown in these figures.

The stone-setting mortar used in the front of the wall was of two kinds. All stones above the base in the east half were set in a 1:3 lime mortar, using a high calcium hydrate. The other parts, including the stonework on the back of the wall, were set in a 1:0.4:3 portland cement, whiting, and sand mortar. All stones set in lime mortar. were wedged at front and back to prevent settling and the wedges were left in place for about a week before removal. The consistency of the mortars was judged by the mason and both mortars had excellent workability. The vertical joints were poured full, and for these a creamy consistency was used. All joints in the south face of the wall were raked out to a depth of 1 inch before the mortar had set. After all stones in this part of the wall had been set, the joints were pointed with mortar of the same mixtures as those used in the setting, i.e., lime mortar for the right half and cement mortar for the rest. A pointing tool of rounded contour was used to obtain slightly concave joints. 
The back of the wall has two set-backs, the tops of which are covered with thin stones forming water tables; the first, or lower table, with two varieties of sandstone flagging, and the second with 37 small marble slabs. Up to the first water table, the back of the wall is concrete except for 23 blocks of granite, which were set in the concrete when it was being poured. Between the first and second water tables, the wall is faced with sandstone and granite. Some of the stone facing on the back is of thin slabs for testing discoloration effects.

The ends of the wall are faced with sandstone, limestone, and granite of miscellaneous sizes and shapes. Under the vertical joints between the marble slabs forming the second water table, thin pieces of slate were set to prevent leakage and discoloration of the stone below.

Wherever metal strips were used to anchor the stone facing to the backing, they were set into the back parts of the stone joints with cement mortar like that used in the left half of the wall. It was necessary to build up the backs of a few thin slabs of stone facing with brick in order to support the thicker blocks above. The locations of these brick back-ups are shown in figure 2 .

The wall was built by the Louis Perna Co. of Washington, D. C., with one mason, Vincent Di Benedeto, setting all the stonework. He was an exceptionally careful workman and took a special interest in doing a thorough job. William Amoroso, who had charge of the work, devised several experiments of his own on the back of the wall. The contractor supplied most of the stone for this face.

\section{Orientation and Exposure Conditions}

The wall faces south, and most of the stonework is exposed to sunlight except for a short time early in the morning and for a somewhat longer period in the afternoon, when it is shaded by trees.

The elevation of the test plot is about $400 \mathrm{ft}$. and the wall is near the top of a hill. Temperatures vary from $-15^{\circ}$ to $106^{\circ} \mathrm{F}$, and the average annual temperature is about $56^{\circ} \mathrm{F}$. Several days of snow and sleet may occur during 5 months of the year, the annual precipitation averaging about 40 in. Humidity is high during the summer months. The exposure plot is about 120 miles from the ocean, hence, salt air is not an appreciable weathering agent. There are few industrial plants within 40 miles, the immediate vicinity being mainly residential. Fuels used for domestic heating are mainly natural gas, oil, and coal. It is believed that the atmospheric polution from these fuels constitutes an important weathering agent.

\section{Arrangement of Stones in Wall}

Most of the stones from the National Museum varied in size from 4- to 12-in. cubes. Figure 2 shows the general arrangement of different sizes, the larger blocks being placed in the lower courses and the smaller ones above. In order to add to the stability and appearance, a sufficient number of larger blocks were collected from present-day producers to construct a granite base with marble quoins on the left, sandstone quoins on the right, and a coping of limestone. As one feature of the test was to determine the performance of stone when set in two types of mortar, the wall was divided into two equal parts with a divider strip of greenstone. For these border blocks, stones from different districts were used insofar as this was feasible. Each block of granite in the base represents a different district, quarry, or distinct grade. The marble quoins represent 5 districts and 11 grades; the sandstone quoins, 7 districts and 9 grades; and the coping blocks 5 districts and 17 grades.

Above course 4, the stones were segregated as to type in order to study the effects of certain combinations. In the two upper end portions, the dolomites and magnesian limestones were used. Below these, calcitic stones were set. In a large area of the top central part, other calcitic stones were placed, and below them siliceous varieties were set in a large area. The stones were set in these combinations in order to study the effects of different mineral compositions on each other, particularly the effect on calcites set under magnesian stones and siliceous materials under calcites.

As a considerable number of colors occur in each type of stone, it was not possible to secure any logical color arrangement.

\section{Problems under Study}

A considerable number of problems involving weathering of various types and varieties of stone, as well as certain structural features of the wall, are being studied. Although all blocks in the wall are subjected to essentially the same external weathering, there are variations in the conditions resulting from: (1) the positions with respect to waterproofing materials, (2) the distance from the ground, (3) the distance from top or ends, (4) the setting of stones in different mortars. There are enough similar stones in various positions to throw some light on all of these problems.

In stone structures, two aspects of weathering are important: (1) color changes, (2) disintegration.

Colors may change because of fading or by becoming concealed by a weathered film on the surface. Discolorations that are caused by a deposit of grime on the surface or stains penetrating from external sources are not usually considered as weathering phenomena.

Among the mole severe weathering agents that ultimately bring about deterioration are frost 


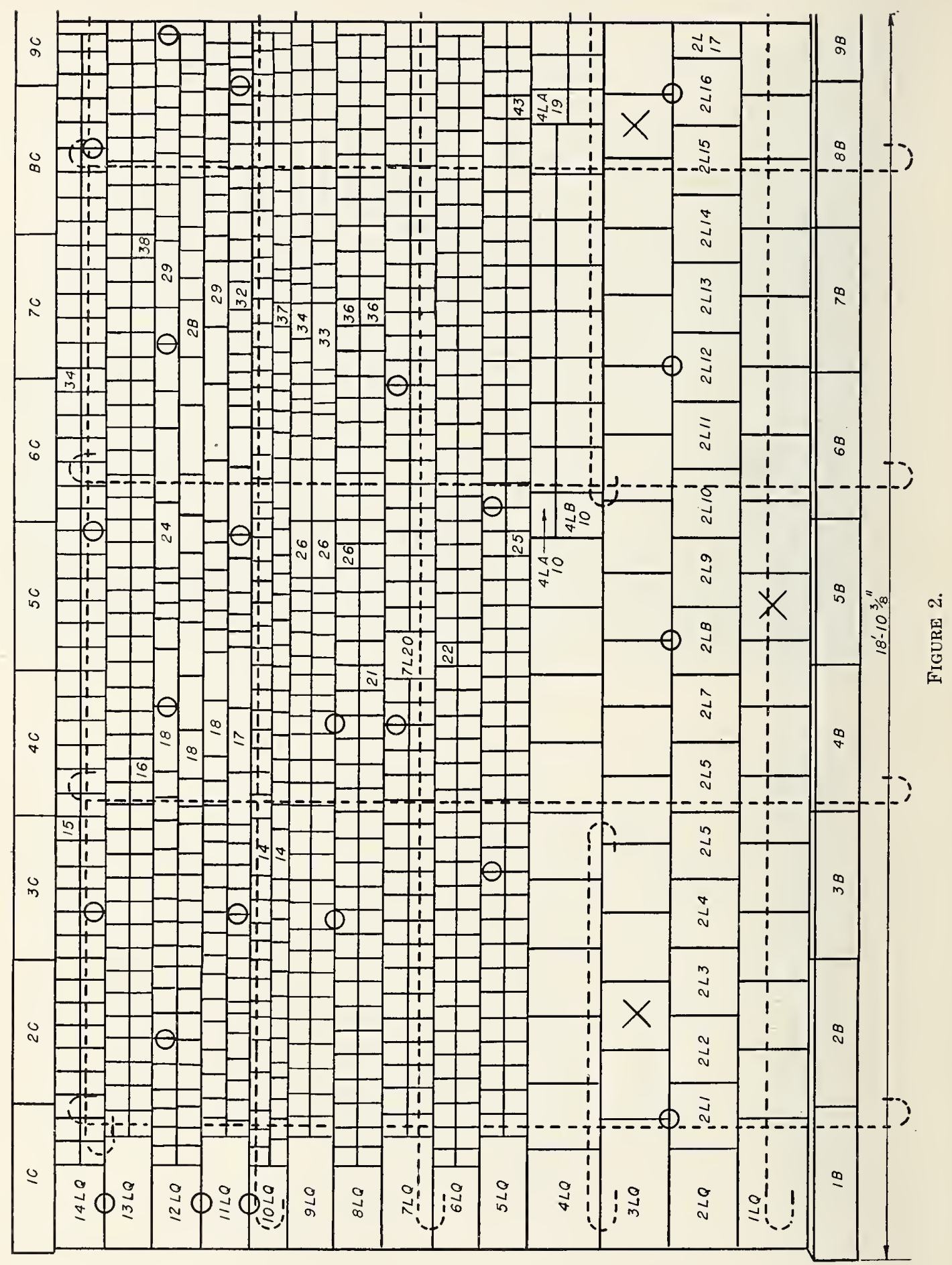




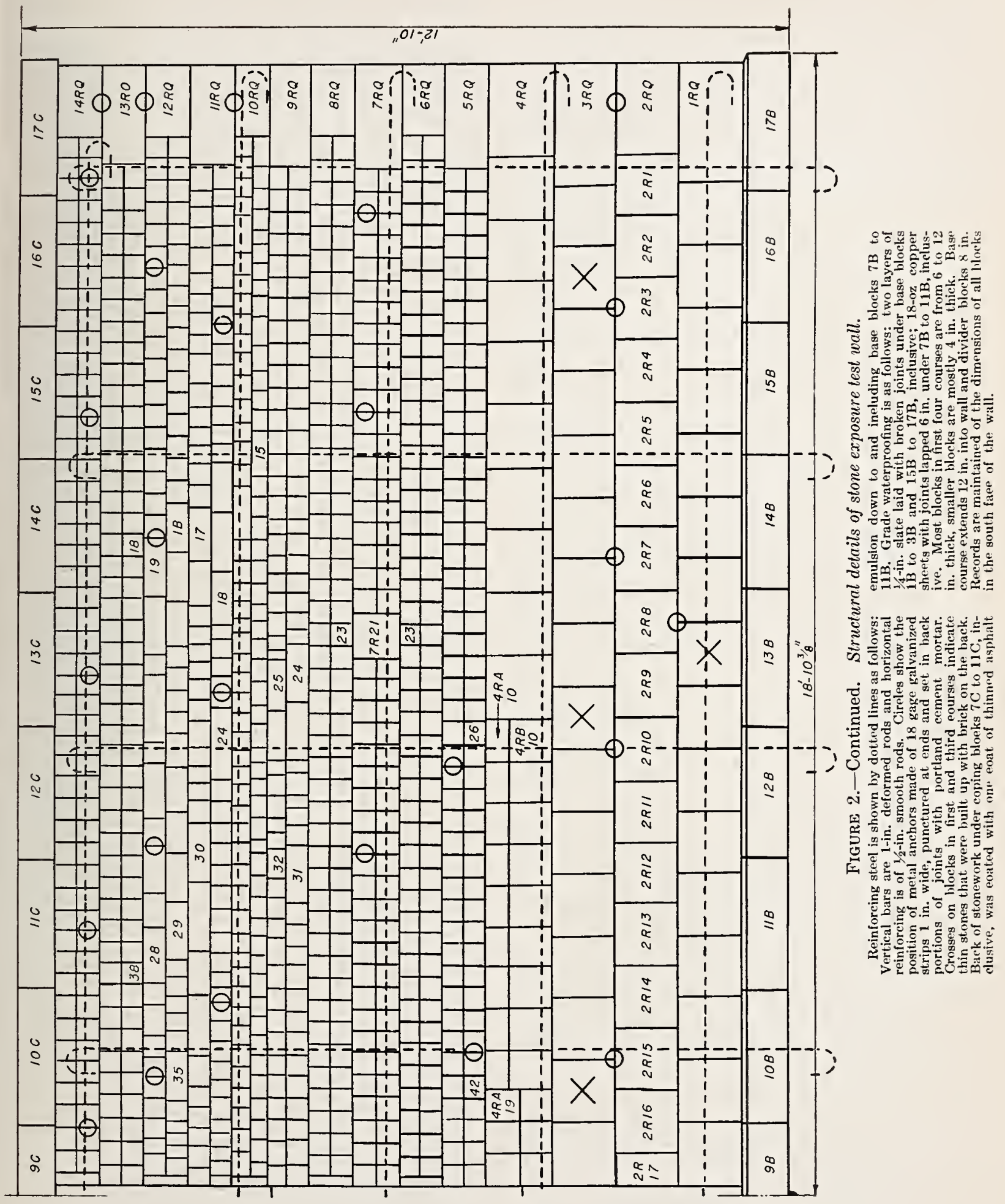


action, temperature changes, crystallization of salts in the pores, atmospheric acids and chemical alterations of the mineral constituents. Chemical changes that result in oxidation, hydration, or sulfation may cause expansion of certain mineral constituents and produce stresses within the stone. Temperature effects are believed to be important for certain crystalline stones that are composed of two or mole minerals having different rates of expansion and also for those stones in which the minerals expand at different rates along different crystallographic directions. Differential expansions between surface layers and the stone at some depth beneath the surface due to moisture or temperature gradients are sometimes thought to accelerate deterioration.

Some of the specific questions that may be answered are as follows:

1. Does the presence of large pores in certain poorly cemented stones, like the shell stones, contribute to frost resistance?

2. Will the rather poorly cemented siliceous stones, like the tuffs, resist the weather conditions of a moderately severe and humid climate?

3. Do dense limestones suffer from the same kinds of weathering as more porous ones?

4. If frost action is the chief weathering agent for sandstone, does the amount of porosity determine the order of durability of such stones?

5. To what extent do the capillary properties of different stones determine the migration of salts in masonry?

6. To what extent does the presence of watersoluble salts in masonry affect different kinds of stone?

7. Does the composition or concentration of salts in masonry influence the rate of weathering?

8. If heating and cooling is the main cause of destruction in marble, do differences in the way individual crystals are joined together determine differences in durability?

9. If the acid condition of the air is the chief cause of weathering for granite, do slight differences in mineral constituents determine the order of durability for different granites?

10. What mineral constituents contribute to weathering by chemical alterations?

11. Do combinations such as calcites and dolomites in masonry result in more rapid weathering than when the masonry is all of one type?

12. What combinations of mineral constituents in stone are unstable because they undergo chemical alterations?

13. What is the nature and source of discolorations?

14. Do iron particles imbedded in the back face of thin granite veneer (deposited from the gang saws) result in discolorations of the exposed face in masonry?

15. To what extent does the nature of the setting mortar affect weathering and discoloration?
16. Does a grade waterproofing under granite near the ground prevent discoloration or weathering of the stone?

17. Does a water barrier between the stone facing and concrete backing reduce discolorations on the stone?

18. How effective is a thin piece of slate under vertical joints in preventing leakage and discolorations on the masonry below?

19. Does thermal expansion of blocks result in permanent or residual changes in dimensions of masonry?

20 . Is stone at the corners of buildings subject to more severe weathering than in the center of a wall?

\section{Methods of Study}

\subsection{Weathering Effects}

Until weathering effects approach destruction, the studies will be confined to surface examinations. After deterioration reaches an advanced stage, the plan is to remove the affected blocks from the wall for various physical, chemical, and microscopic studies to determine the cause and extent of weathering. There is evidence that stones differing considerably in composition or physical makeup may suffer deterioration from quite different kinds of weather action. The most important phase of the study is to identify the principal causes of deterioration for different groups with respect to mineral composition, physical structure, porosity, capillary properties, etc.

\subsection{Color Changes}

Discolorations on masonry include the following variations: (1) accumulation of grime on the surface, (2) stains penetrating from exterior sources, (3) stains originating in the masonry itself, and (4) changes in color due to weathering processes.

It is expected that some useful information on these phases of the subject can be obtained by observations and comparisons. It may also be desirable to determine, by experiment, whether or not various kinds of discoloration can be easily removed.

A large portion of the stonework set in each kind of mortar was entirely free from stain although occasional blocks stained badly in a short period of time and streaked downward on the mortar and stones below. Such performance seems to indicate that the staining matter exuded from the stone. Where a sufficient amount of the original sample is available, experiments may determine what conditions will reproduce the stain. If the stain can be reproduced by leaching the sample with weak alkaline solutions, it will be evident that the staining matter is in the stone itself. 


\subsection{Dimensional Changes}

There is evidence that thermal expansion may cause permanent increases in the over-all dimensions of masonry. In order to study the changes in dimensions of various parts of the wall due to weathering, reference plugs were set into the base, the coping, and courses 2,4 , and 9 . The plugs are made of brass round, $1 / 2$ in. in diameter and 2 in. long, and are threaded the entire length. They are set with cement mortar into 1-in. holes drilled in the stone with a diamond-core drill. A $1 / 8$-in. hole in each plug serves as a reference mark. The ends of the plugs are flush with the exposed face of the stonework, but the mortar holding them in place is recessed about 1/4 in. Brass caps are used to protect the ends of the reference plugs. The length of the base course is carefully measured with a precision steel tape, and that of higher courses is obtained with a transit. The transit is set in front of each vertical line of plugs, leveled, and the vertical cross wire set on the left edge of the hole in the base plug. The telescope is then raised to each reference mark above, and the distances from the cross wire to the reference mark read on a millimeter scale. The lengths of the upper courses can then be computed. Comparisons of subsequent measurements with the original will show whether changes have occurred.

\subsection{Warpage}

If differential expansions between the facing and backing materials occur, the wall will probably warp. To determine whether warpage is taking place and to what extent, periodic measurements are being made. For this, a transit and two especially constructed pins, inserted into the holes of the reference plugs at the ends of course 2, are used. The smaller portion of each pin fits snugly into the hole of the reference plug and the larger, or projecting portion, is notched at a distance of $1 \%$ in. from the shoulder that forms the junction between the two parts of the pin. The transit is set up in line with the notches about 15 $\mathrm{ft}$. from one end of the wall. The vertical wire of the telescope determines a plane parallel to the face of the wall. A millimeter scale with the zero end held on the center of various blocks in courses 2,4 , and 7 is read through the transit and the distances recorded. Comparison of subsequent readings with the original indicates the amount of warpage.

\section{Maintenance of Records}

A sealed box, made of stainless steel, is set into a vault in the back of the wall for safe storage of records. In addition to the original records set forth in this paper, certain other information of possible use will be filed therein. Photographs of the wall, showing the original appearance by closeup views and color reproductions, as well as copies of subsequent observations, measurements, and test data, will be stored in this box. Small samples of many stones in the face of the wall will be stored for future comparison with the weathered stones.

\section{Catalog of Stones in Wall}

\subsection{Explanation of tables 1 and 2}

The listing of domestic samples in table 1 is intended to facilitate the location of specific materials in the wall when the source and type are known. The arrangement follows the alphabetical order: first, of States supplying the samples; second, of types under each State; and third, of specific source names that are shown in column 1. The left (west) half of the south face is indicated in the wall numbers by the $\mathrm{L}$ and the right half (east) by R. The listing of foreign stones in table 2 follows the same arrangement. The foreign stones were more limited in number than the domestic, and no attempt was made to subdivide the countries into political subdivisions, although provinces are stated when known.

To identify a particular stone in the wall when the type and source are known, for instance, "Golden Vein Yule" marble from Marble, Colo., table 1 should be consulted by first locating the state, then the type (in this case marble) and finally under this type group, the name of the town that supplied the sample. Opposite the name of the town, six wall numbers appear, the last two being $1 \mathrm{~L} 13$ and $1 \mathrm{R} 13$. The first number signifies the course, counting from the base upward, L13 indicates the 13th block from the left, and R13 the 13th block from the right. In counting from either left or right, the quoin (end stone) should be disregarded. The number of courses is determined by the quoins, there being 14 courses in addition to the base course and coping (top course). Above course 4, there are two rows of small stones to the course, the upper one being designated $\mathrm{A}$ and the lower one $\mathrm{B}$. Thus, 5LA7 means the 7th block from the left quoin of course 5 upper row, and $6 \mathrm{RB} 9$, the 9 th stone from the right quoin in course 6 , lower row. Base blocks are designated $1 \mathrm{~B}$ to $17 \mathrm{~B}$, numbered from left to right. The coping blocks are likewise designated $1 \mathrm{C}$ to $17 \mathrm{C}$ in the same order. Quoins on the left are designated $1 \mathrm{QL}$ to $14 \mathrm{QL}$, and those on the right, $1 \mathrm{QR}$ to $14 \mathrm{QR}$, numbered from bottom to top. The divider strips are called $1 \mathrm{D}, 2 \mathrm{D}$, and $3 \mathrm{D}$, from bottom to top.

An asterisk after the description of a stone indicates that it has been used for building or other purposes. Important trade names are stated and enclosed in quotation marks.

\subsection{Explanation of table 3}

To identify a particular stone in the wall when the type and source are not know, it will be necessary to determine the wall number by actual 
count. When this number is found (e.g., 7RB35), table 3 should be consulted. This number can be found easily, because the courses and wall numbers are listed in sequence. Opposite the wall number the designation "conglomerate from Massachusetts" appears. More complete information may be found in table 1 under Massachusetts conglomerates, where the sample is described as "Pudding Stone" from Dorchester.

In determining the wall numbers, it will often be helpful to consult fig. 2 . In this figure, several of the more prominent blocks show the count numbers, which may be used to avoid counting from the end blocks. It should be remembered that these numbers are obtained by starting with the block next to the quoin and counting toward the middle.

In table 3, the base, coping, quoin, and divider stones are listed after those in courses 1 to 14 .

\subsection{Stones in Back and Ends of Wall}

Stones in the back face and ends of the wall are of random sizes and were not set in continuous courses like those in the south face. The identi- fication numbers of the blocks are shown in figure 3 , and descriptions are given in table 4 . In order to simplify the process of finding specific materials in the wall, the locations are divided into 5 sections: section $\mathrm{S}-1$ includes all blocks in and below the first or lower water table except those numbered in the end sections; section S-2 includes all stones in and below the second water table down to section $\mathrm{S}-1 ; \mathrm{S}-3$ includes all above the second water table but not the coping stones. The end stones include those showing on the ends and not numbered on the south face. Those blocks that can be seen on the ends and also on the back are numbered only as end stones. Blocks on the east end are given consecutive numbers starting from the base and followed by the letters EE, and those on the west end are numbered in the same way, followed by the letters WE.

Some of the stones on the back of the wall were given certain treatments on the back surfaces before setting. Such stones are designated by numbers in parenthesis following the wall numbers. Notes in the legends of figure 3 describe the treatments.

TABLE 1. Source, classification, and description of domestic stones in south face of wall

[For identification of samples in wall see section 8, 1. Geological terms are capitalized, trade names are enclosed in quotation marks, and a star (*) at end of line signifies that the stone has been used commercially.]
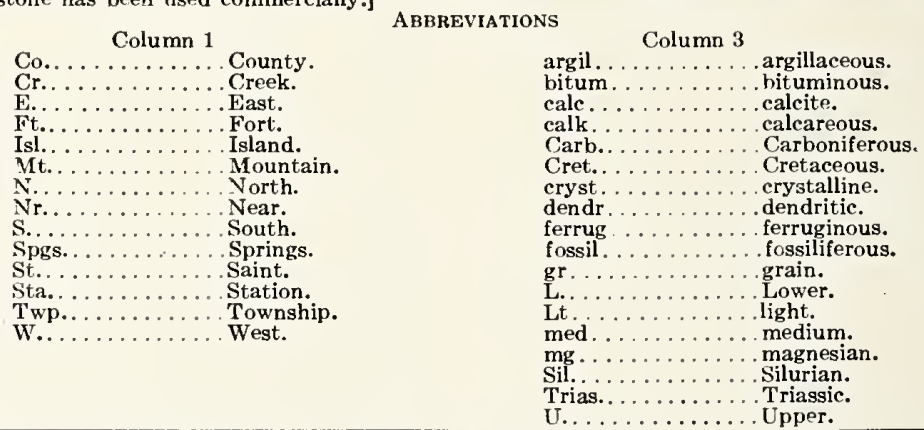

\begin{tabular}{|c|c|c|c|c|c|}
\hline $\begin{array}{l}\text { Specific } \\
\text { source }\end{array}$ & $\begin{array}{l}\text { Wall } \\
\text { No. }\end{array}$ & Description & $\begin{array}{l}\text { Specific } \\
\text { source }\end{array}$ & $\begin{array}{l}\text { Wall } \\
\text { No. }\end{array}$ & Description \\
\hline \multirow{2}{*}{\multicolumn{3}{|c|}{$\begin{array}{l}\text { Alabama Limestone } \\
\text { See also } \mathrm{S}-3,4 \text { and } 5 \mathrm{EE} \text { in table } 4\end{array}$}} & \multicolumn{3}{|c|}{ Alabama Sandstone } \\
\hline & & & & & \multirow{2}{*}{$\begin{array}{l}\text { Yellow, coarse-gr, porous, ferrug.* } \\
\text { Gray, fine-gr, compact.* }\end{array}$} \\
\hline \multirow{4}{*}{ 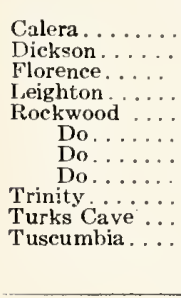 } & \multirow{4}{*}{$\begin{array}{l}\text { 12LB39 } \\
\text { 7RA8 } \\
\text { 7LA7 } \\
\text { 13RB46 } \\
\text { 2C } \\
\text { 16C } \\
\text { 13RA47 } \\
\text { 13LA47 } \\
\text { 12LA37 } \\
\text { 12RB40 } \\
\text { 7RA9 }\end{array}$} & \multirow{4}{*}{$\begin{array}{l}\text { Bluish-gray, fine gr, compact, L. Sil. } \\
\text { It-gray, med-gr, compact, fossil. } \\
\text { Lt-gray, coarse-gr, compact, cryst. } \\
\text { Lt-gray, med-gr, porous, fossil. } \\
\text { Lt-gray, fine-gr, porous, oolitic.* } \\
\text { Lt-buff, fine-gr, porous, oolitic.* } \\
\text { Lt-gray, fine-gr, porous, oolitic.* } \\
\text { Cut from same block as 13RA47. } \\
\text { Ltt-gray, coarse-gr, conpact, semicryst. } \\
\text { Lt-gray, coarse-gr, porous, tertiary. } \\
\text { Lt-gray, med-gr, compact, fossil- } \\
\text { bearing.* }\end{array}$} & Greensport........ & 9LB36 & \\
\hline & & & \multicolumn{3}{|c|}{$\begin{array}{c}\text { Alaska Marble } \\
\text { See samples } \mathrm{S}-2,145 \text { and } \mathrm{S}-2,151 \text { of table } 4\end{array}$} \\
\hline & & & \multicolumn{3}{|c|}{ Arizona Marble } \\
\hline & & & 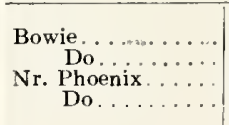 & $\begin{array}{l}\text { 14RB24 } \\
\text { 14LB23 } \\
\text { 10LA22 } \\
\text { 10RA21 }\end{array}$ & $\begin{array}{l}\text { White, dark-veined, coarsely cryst. } \\
\text { Do. } \\
\text { Lt drab and yellow mottled ony } x \text {. } \\
\text { Cut from same block as 10LA22. }\end{array}$ \\
\hline \multicolumn{3}{|c|}{$\begin{array}{c}\text { Alabama Marble } \\
\text { (See also } \mathrm{S}-2,152 \text { and } \mathrm{S}-2,167 \text { of table } 4 \text { ) }\end{array}$} & \multicolumn{3}{|c|}{ Arizona Sandstone } \\
\hline \multirow{3}{*}{ 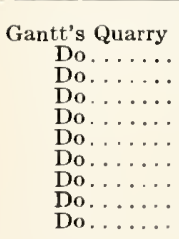 } & \multirow{3}{*}{$\begin{array}{l}2 \mathrm{LQ} \\
4 \mathrm{LQ} \\
4 \mathrm{LA} 10 \\
4 \mathrm{RA} 10 \\
12 \mathrm{LB} 38 \\
12 \mathrm{~L} 40 \\
12 \mathrm{RA} 37 \\
12 \mathrm{RB} 39 \\
12 \mathrm{R} 41 \\
13 \mathrm{LB} 46\end{array}$} & \multirow{3}{*}{$\begin{array}{l}\text { White, fine-gr calc, "Cream A".* } \\
\text { White, fine-gr calc, "Pentellic A".* } \\
\text { White, dark parallel veins, fine-gr calc.* } \\
\text { Cut from same block as 4LA10. } \\
\text { White, dark parallel veins, fine-gr, calc.* } \\
\text { Cut from same block as } 12 \mathrm{LB} 38 . \\
\text { Do. } \\
\text { Do. } \\
\text { Do. } \\
\text { Do. }\end{array}$} & 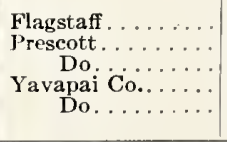 & $\begin{array}{l}\text { 8LA38 } \\
\text { 9LA33 } \\
\text { 9RA35 } \\
\text { 9RB33 } \\
\text { 8LB38 }\end{array}$ & $\begin{array}{l}\text { Red, fine-gr, compact.* } \\
\text { Red, coarse-gr, compact.* } \\
\text { Do. } \\
\text { It-red, fine-gr, compact.* } \\
\text { Pinkish, fine-gr, porous.* }\end{array}$ \\
\hline & & & \multicolumn{3}{|c|}{ Arizona Volcanic Tuff } \\
\hline & & & $\begin{array}{l}\text { Kirkland. } \\
\text { Do. . }\end{array}$ & $\begin{array}{l}\text { 8RA38 } \\
\text { 7LA36 }\end{array}$ & $\begin{array}{l}\text { Gray, fine-gr, volcanic tuff.* } \\
\text { Do. }\end{array}$ \\
\hline
\end{tabular}


TABLE 1. Source, classification, and description of domestic stones in south face of wall-Continued

\begin{tabular}{|c|c|c|}
\hline $\begin{array}{l}\text { Specific } \\
\text { source }\end{array}$ & $\begin{array}{l}\text { Wall } \\
\text { No. }\end{array}$ & Description \\
\hline \multicolumn{3}{|c|}{$\begin{array}{c}\text { Arkansas Limestone } \\
\text { Sce also } \mathrm{S}-3,7,10,26 \mathrm{EE} \text {, and } 32 \mathrm{WE} \text { of table } 4\end{array}$} \\
\hline Batesville........ & $11 \mathrm{RB} 22$ & Grayish-whitc, fine-gr, compact. \\
\hline \multicolumn{3}{|c|}{ Arkansas Marble } \\
\hline $\begin{array}{l}\text { Yr. Batesville } \\
\text { Batesville .... } \\
\text { Sugar Orchard } \\
\text { Cr., Boone Co. . }\end{array}$ & $\begin{array}{l}\text { 7RA10 } \\
10 \mathrm{LQ} \\
7 \mathrm{LA} 8\end{array}$ & $\begin{array}{l}\text { Nearly black, fine-gr, calc. } \\
\text { Mottled gray and white, fine-gr, calc.* } \\
\text { Dull-brown, white-spotted, crinoidal.* }\end{array}$ \\
\hline
\end{tabular}

Arkansas Quartzite

\begin{tabular}{l|l|l}
\hline Bald Knob ...... & $11 \mathrm{LB} 27$ & Buff, fine-gr, compact.* \\
\hline
\end{tabular}

California Basalt

\begin{tabular}{l|l|l} 
Bridgeport....... & 5LB29 & Gray, fine-gr, compact.* \\
Petaluma....... & 5RA29 & Dark-gray, fine-gr, compact.*
\end{tabular}

California Granite

\begin{tabular}{l|l|l} 
Penryn........ & 5RA30 & $\begin{array}{l}\text { Dark-olive, black-spotted, med-gr, } \\
\text { hornblende** } \\
\text { Gray, fine-gr, biotite** }\end{array}$ \\
Rocklin......... & 5LA29
\end{tabular}

California Marble

See also S-2, 142 to 144 of table 4

\begin{tabular}{l|l|l}
\hline Colfax........ 12LA2 & Dark-gray, fine-gr, mg.* \\
\hline
\end{tabular}

California Sandstone

\begin{tabular}{|c|c|c|}
\hline 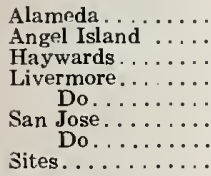 & $\begin{array}{l}\text { 6RB30 } \\
\text { 7RA29 } \\
\text { 6RA31 } \\
\text { 7LB29 } \\
\text { 7LA29 } \\
\text { 6LA31 } \\
\text { 6LB31 } \\
\text { 7RB28 }\end{array}$ & $\begin{array}{l}\text { Lt-brown, coarse-gr, porous. } \\
\text { Gray, fine-gr, porous.* } \\
\text { Brown, med-gr, porous.* } \\
\text { Gray, fine-gr, porous.** } \\
\text { Gray, fine-gr, compact. } \\
\text { Buff, coarse-gr, compact.* } \\
\text { Pink, med-gr, compact.** } \\
\text { Greenish-gray, fine-gr, porous. }\end{array}$ \\
\hline
\end{tabular}

California Tuff

\begin{tabular}{l|l|l}
\hline Cordelia......... & $5 \mathrm{LB} 28$ & Gray, coarse andesite.* \\
Lordsburg....... & $5 \mathrm{RB30}$ & Dull-red, fine-gr, compact, basalt.*
\end{tabular}

Colorado Diorite

\begin{tabular}{l|l|l}
\hline Monarch........ & 5RB32 & Greenish-gray, fine-gr.* \\
\hline
\end{tabular}

Colorado Granite

See also $\mathrm{S}-2,53$, and $\mathrm{S}-2,100$ of table 4

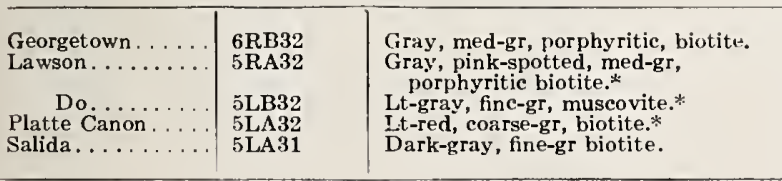

Colorado Limestone

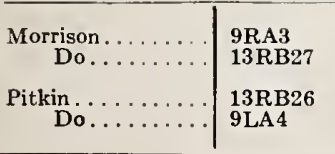

Lt-red, finc-gr, porous, laminated.*

Dull-pink, coarse-gr, compact,

senicryst.*

Black, fine-gr, compact.*

Dark, white-mottled, fine-gr, compact.

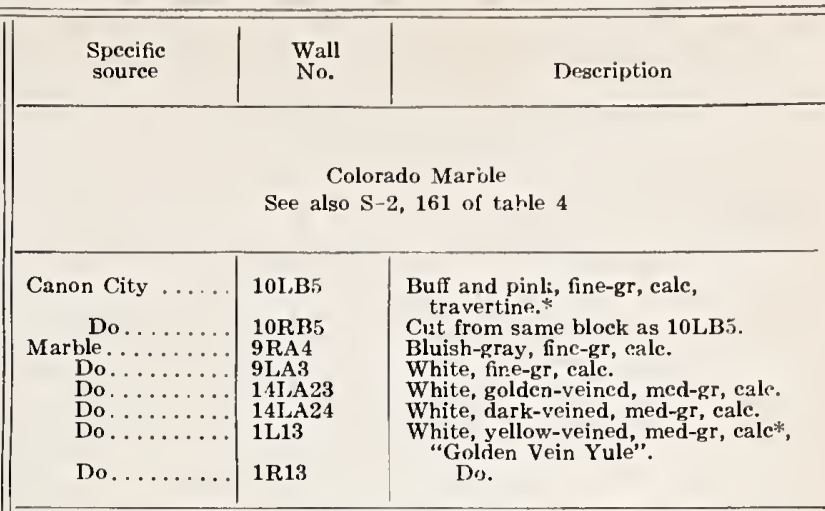

Colorado Quartzite

\begin{tabular}{l|l|l} 
Pitkin ......... & 5LB31 & White, very fine and compact.*
\end{tabular}

Colorado Sandstone

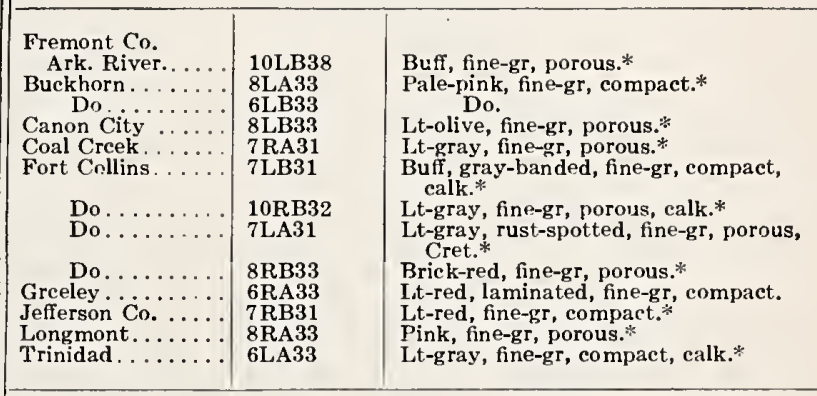

Colorado Tuff

\begin{tabular}{l|l|l} 
Castle Rock..... & 6 RB33 & I.t-gray, fine-gr, rhyolite.*
\end{tabular}

Connecticut Conglomerate

\begin{tabular}{|l|l|l}
\hline Portland ........ & 5RA34 & Brownish-purple, porous, Trias.*
\end{tabular}

Connecticut Diabase

\begin{tabular}{l|l|l}
\hline New Haven...... & 5 RB35 & Darli-gray, fine-gr, Mesozoic.*
\end{tabular}

Connecticut Gneiss

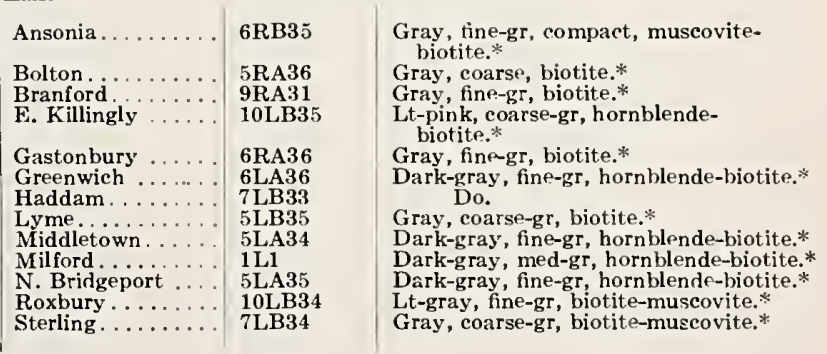


TABLE 1. Source, classification, and description of domestic stones in south face of wall-Continued

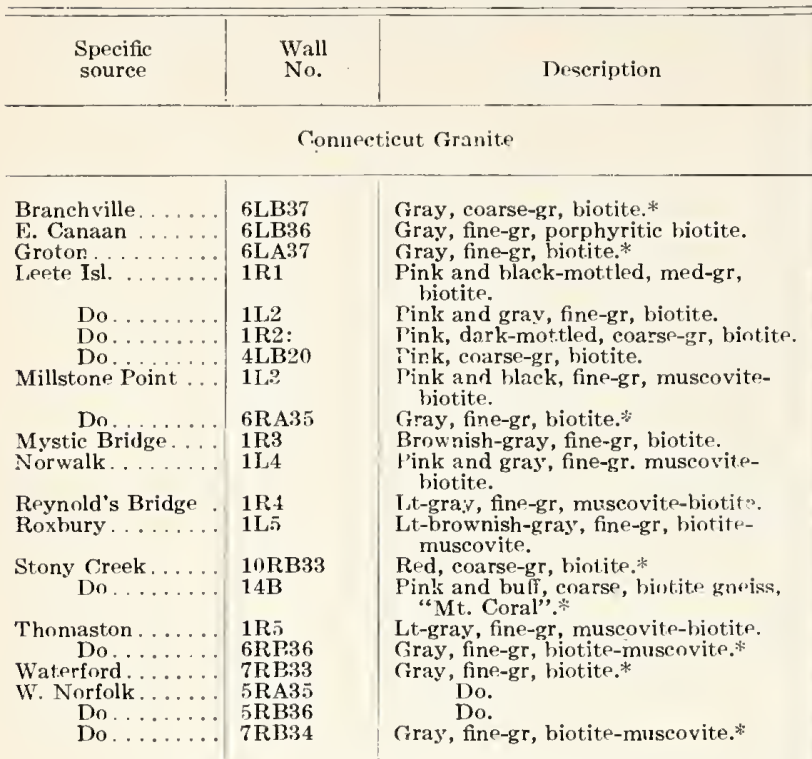

\section{Connecticut Limestone}

\begin{tabular}{r|l|l}
$\begin{array}{r}\text { Shetlerville...... } \\
\text { Do ....... }\end{array}$ & 7LA9 & $\begin{array}{l}\text { Drab, med-gr, compact. } \\
\text { Dark-gray, fine-gr, compact. }\end{array}$ \\
\hline
\end{tabular}

Connecticut Marble

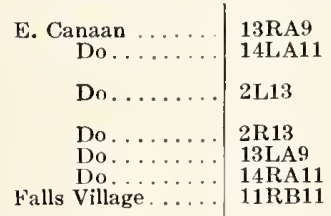

\begin{abstract}
Pluish-white, coarse-gr, dolomite.* White, dark-clouded, coarse-gr, dolomite.*

White, brown-spotted, coarse-gr, dolomite.

Cut from same block as $2 \mathrm{~L} 13$. Water-blue, granular dolomite.*

Do.
White, granular-cryst dolomite.*
\end{abstract}

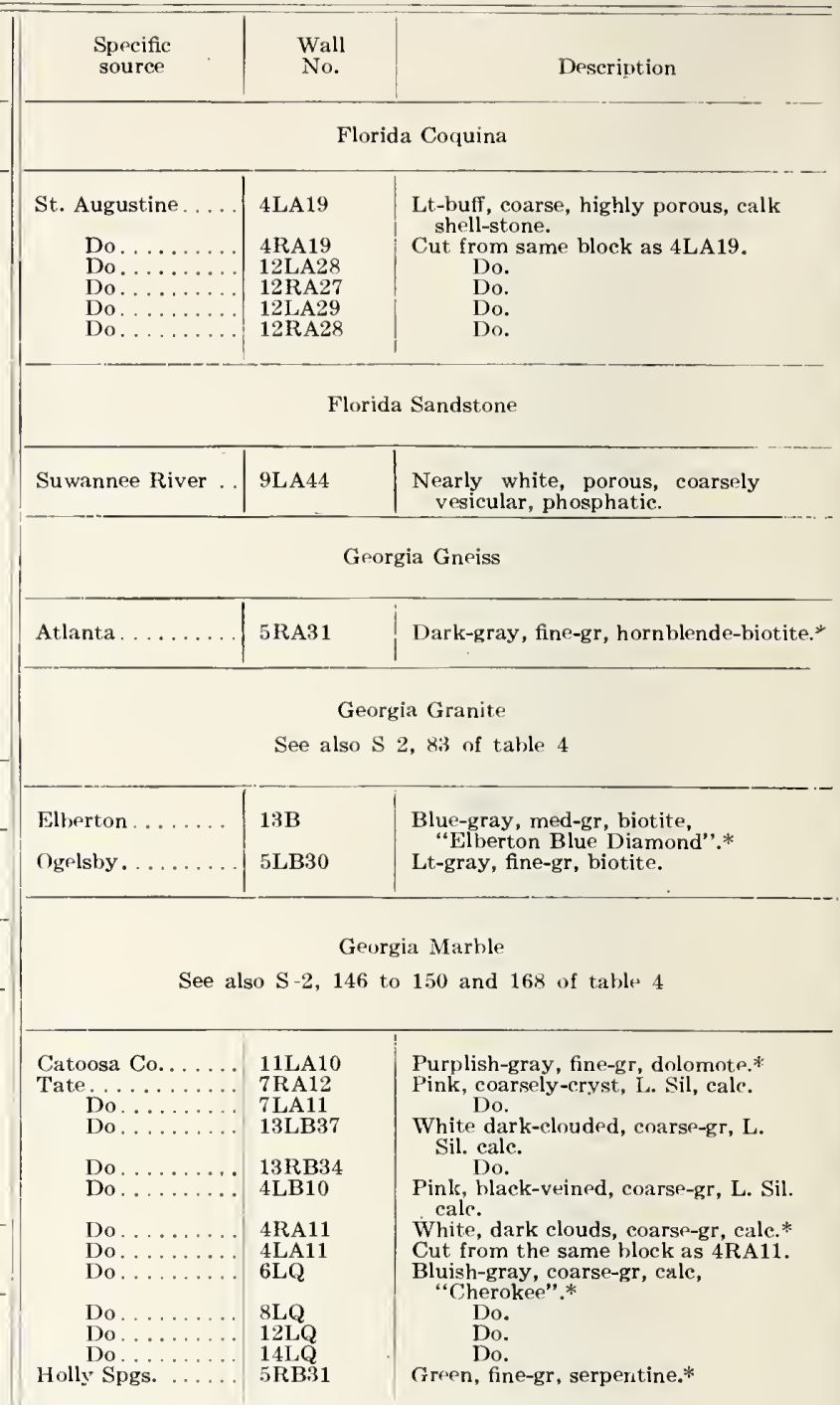

Idaho Sandstone

See also $\mathrm{S} \cdot 2,8, \mathrm{~S} 2,132$, and $23 \mathrm{EE}$ of table 4

\begin{tabular}{|l|l|l|}
\hline Boise ........ & 9RA43 & Buff-colored, coarse-gr, porous.* \\
\hline
\end{tabular}

Illinois Dolomite

Delaware Gneiss

\begin{tabular}{r|c|c}
$\begin{array}{r}\text { Wilmington } \ldots \ldots \ldots \\
\text { Do...... }\end{array}$ & $\begin{array}{l}5 \mathrm{RB} 46 \\
\text { 4RB16 }\end{array}$ & $\begin{array}{c}\text { Dark-gray, fine-gr, augite-hornblende.* } \\
\text { Greenish-gray, fine-gr, augite- } \\
\text { hornblende. } \\
\text { Dark-gray, mediural-gr. }\end{array}$ \\
\hline
\end{tabular}

Delaware Marble

\begin{tabular}{l|l|l}
\hline Hockessin . . . . & 11RA9 & White, coarse-gr, dolomite.*
\end{tabular}

Florida Limestone

See also $\mathrm{S}-2,129$, and $27 \mathrm{EE}$ of table 4

Key West...... 7LA10

Nearly white, porous, friable, oolitic.

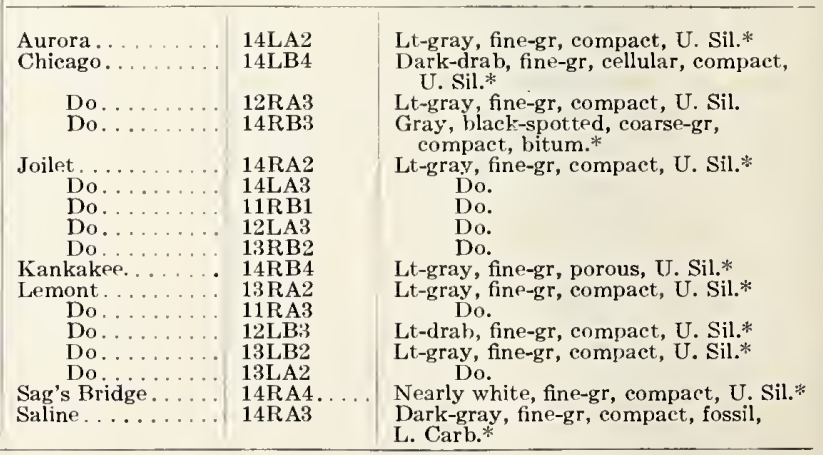


TABLE 1. Source, classification, and description of domestic stones in south face of wall-Continued

\begin{tabular}{|c|c|c|}
\hline $\begin{array}{l}\text { Specific } \\
\text { source }\end{array}$ & $\begin{array}{l}\text { Wall } \\
\text { No. }\end{array}$ & Deseriytion \\
\hline \multicolumn{3}{|c|}{ Illinois Limestone } \\
\hline Bclknap. & 12RB34 & Greenish-gray, fine-gr, compact.* \\
\hline $\begin{array}{l}\text { Do } \\
\text { Breese. }\end{array}$ & $\begin{array}{l}\text { 8LA11 } \\
12 \mathrm{RB} 4\end{array}$ & $\begin{array}{l}\text { Gray, medium-gr, compact.** } \\
\text { Dark-gray, fine-gr, compact, fossil, }\end{array}$ \\
\hline Chester & $13 R B 40$ & Gray, dark-strcaks, med-gr, compact, \\
\hline Do & 8RA10 & $\begin{array}{l}\text { tossil, L. Carb.* } \\
\text { Lt-gray med-gr, finely fossil, compart, }\end{array}$ \\
\hline Columbia . & 13RB39 & $\begin{array}{l}\text { L. Carl,** } \\
\text { I.t-gray, fine-gr, porous, fossil-bearing, }\end{array}$ \\
\hline Iuka ..... & 13LB42 & $\begin{array}{l}\text { L. Carb.* } \\
\text { Gray, fine-gr, compact.* }\end{array}$ \\
\hline & 12LA31 & Lt-gray, fine-gr, compact, L. Sil.* \\
\hline Do $\ldots$ & 12RA30 & Cut from the same block as $12 \mathrm{LA} 31$. \\
\hline Jonesborough & 8RA12 & $\begin{array}{l}\text { Lt-gray, med-gr, compact, oolitic.* } \\
\text { Do. }\end{array}$ \\
\hline Kinderhook : & $8 \mathrm{LA} 12$ & Lt-gray, med-gr, compact, semieryst, \\
\hline Quincy... & 13LA42 & Yellow, fine-gr, compact, L. Carb. \\
\hline $\begin{array}{l}\text { Do.. } \\
\text { Rockport. }\end{array}$ & $\begin{array}{l}\text { 12RA4 } \\
\text { 8RA11 }\end{array}$ & $\begin{array}{l}\text { Lt-gray, coarse-gr, compact, mg, fossil } \\
\text { Lt-gray, med-gr, compact, }\end{array}$ \\
\hline Sagetown. & $13 \mathrm{RB} 38$ & Lt-gray, fine-gr, compact. \\
\hline
\end{tabular}

Illinois Sandstone

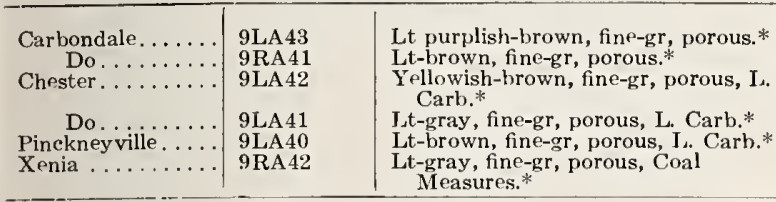

Indiana Dolomite

\begin{tabular}{|c|c|c|}
\hline $\begin{array}{l}\text { Anderson. } \\
\text { Bluffton... } \\
\text { Decatur ... } \\
\text { Eaton.... }\end{array}$ & $\begin{array}{l}\text { 14RA1 } \\
\text { 14RB1 } \\
\text { 12RB1 } \\
\text { 12LB1 }\end{array}$ & $\begin{array}{l}\text { Drab, fine-gr, compact, Niagara }{ }^{*} \\
\text { Gray, fine-gr, compact, Devonian.* } \\
\text { Drab, fine-gr, compact, U. Sil.* } \\
\text { Dark, mottled, coarse-gr, compact, } \\
\text { Niagara.* }\end{array}$ \\
\hline $\begin{array}{l}\text { Greensburgh } \\
\text { Logansport... } \\
\text { Longwood... }\end{array}$ & $\begin{array}{l}\text { 12LA1 } \\
\text { 14LA1 } \\
11 \mathrm{LB1} 1\end{array}$ & $\begin{array}{l}\text { Lt-gray, fine-gr, compact, U. Sil. } \\
\text { Lt-drah, fine-gr, compact, Niagara.* } \\
\text { Yellowish, mottled, fine-gr, compact, } \\
\text { cellular, }\end{array}$ \\
\hline $\begin{array}{l}\text { Montpelier. } \\
\text { St. Paul .... } \\
\text { Wabash.... }\end{array}$ & $\begin{array}{l}\text { 14LB1 } \\
\text { 11LA1 } \\
\text { 11RA1 }\end{array}$ & $\begin{array}{l}\text { Lt-gray, fine-gr, compact, Niagara.** } \\
\text { Lt-gray, fne-gr, compact, Niagara. } \\
\text { It-yellowish, fine-gr, compact, } \\
\text { Niagara. }\end{array}$ \\
\hline
\end{tabular}

Indiana Limestone

See also $S-1, S-2, S-3, E E$ and WE of table 4

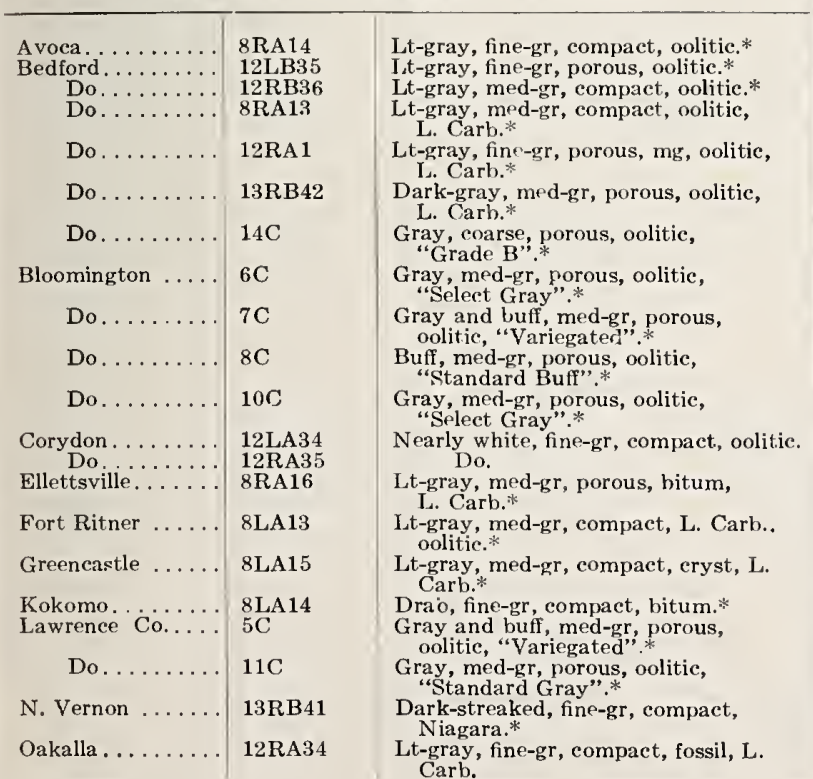

\begin{tabular}{|c|c|c|}
\hline $\begin{array}{l}\text { Specifie } \\
\text { source }\end{array}$ & $\begin{array}{l}\text { Wall } \\
\text { No. }\end{array}$ & Deseription \\
\hline $\begin{array}{l}\text { Oakdale } \\
\text { Owens Co. }\end{array}$ & ${ }_{6 \mathrm{C}}^{14 \mathrm{RB} 12}$ & $\begin{array}{l}\text { Lt-gray, fine-gr, compact, Niagara.* } \\
\text { Gray, med-gr, porous, oolitie, }\end{array}$ \\
\hline Do. & $12 \mathrm{C}$ & Buff, med-gr, celiular oolitic, \\
\hline Do. & $13 \mathrm{C}$ & Buff, coarsersulf rellular, oolitic, \\
\hline Putnamville. & $8 \mathrm{RA} 15$ & Gray, fine-gr, compact, L. Carb., \\
\hline $\begin{array}{l}\text { Salem.. } \\
\text { Do. }\end{array}$ & $\begin{array}{l}\text { 12LB36 } \\
\text { 12RA32 }\end{array}$ & $\begin{array}{l}\text { Dark, fine-gr, compact, L. Carb. } \\
\text { Lt-gray, med-gr, porous, L. Carb., }\end{array}$ \\
\hline Do. & $4 \mathrm{RB} 12$ & Lt-gray, med-gr, porous, L. Carb., \\
\hline $\begin{array}{r}\text { Do. } \\
\text { Spencer. } \\
\text { Do. }\end{array}$ & $\begin{array}{l}4 \mathrm{LB} 12 \\
12 \mathrm{RA} 33 \\
12 \mathrm{LA} 35\end{array}$ & $\begin{array}{l}\text { Do. } \\
\text { Lt-gray, fine-gr, compact, J.. Carh.* } \\
\text { Lt-gray, med-gr, porous, L. Carb., }\end{array}$ \\
\hline Stinesville. & 10RB14 & $\begin{array}{l}\text { bitum.* } \\
\text { Lt-gray, med-gr, porous, fossil- } \\
\text { bearing, bitum.* }\end{array}$ \\
\hline
\end{tabular}

Indiana Sandstone

\begin{tabular}{|c|c|c|}
\hline Attic & 9RB40 & Lt-gray, fine-gr, porous.* \\
\hline Cannelton .......... & 9 LA38 & Lt-brown, fine-gr, porous.* \\
\hline French Lick Twp.. & 9LB40 & Nearly white, fine-gr, porous, \\
\hline & 9LA39 & Lt-gray, fine-gr, porous, L. Carb.* \\
\hline $\begin{array}{l}\text { Paoli........ } \\
\text { Williamsport }\end{array}$ & $\begin{array}{l}\text { 9RB39 } \\
9 \mathrm{LB} 41\end{array}$ & $\begin{array}{l}\text { Lt-gray, fine-gr, porous, I. Carh. } \\
\text { Cream-colored, med-gr, porous, Carb.* }\end{array}$ \\
\hline
\end{tabular}

Towa Coral

Iowa City ...... 13 LA20 Lt-gray, fine-gr, compact, fossil.

Iowa Dolomite

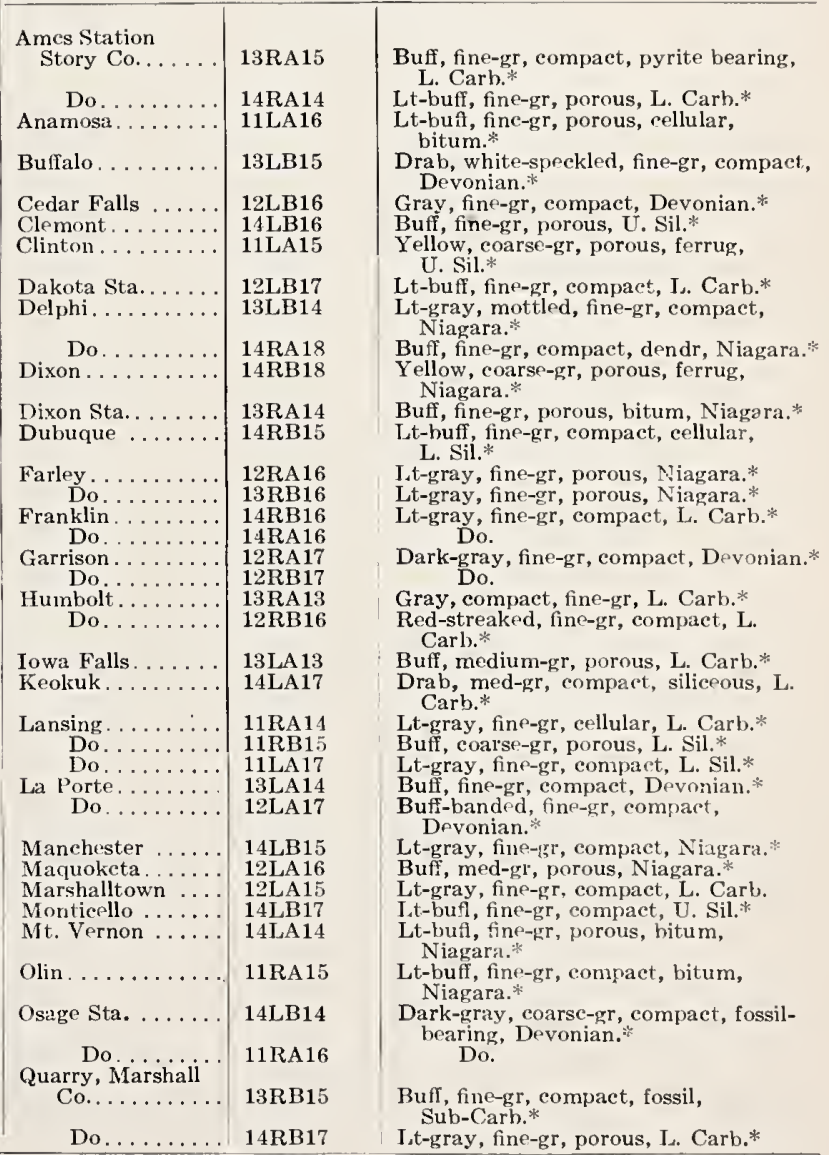


TABLE 1. Source, classification, and description of domestic stones in south face of wall Continued

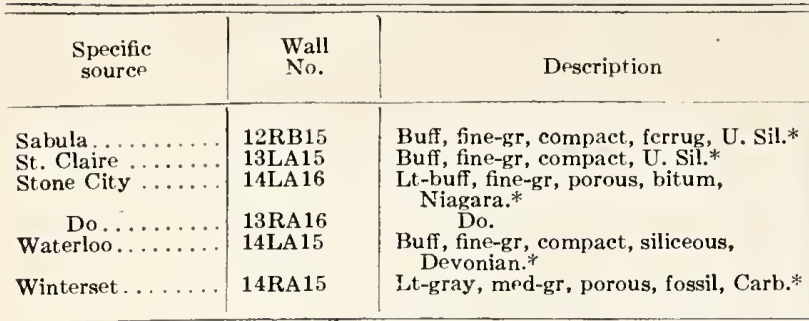

Iowa Limestone

\section{Bedford.
Do \\ Bentonsport \\ Blansett \\ Bristow. \\ Do...... \\ Burlington \\ Do \\ Charles City \\ Do... \\ Conrad.... \\ Corning. \\ Davenport \\ Do.... \\ Do. \\ Dudley Sta. \\ Wapello Co. \\ Do. \\ Durham Sta \\ Marion Co. \\ Earlham. \\ Do. . \\ Fairfield.}

Fort Dodge

Givin..

Independence.

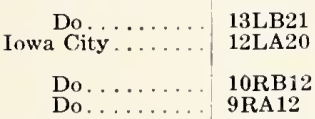

Jowa Falls

Do

Kena....... 10LA20

Le-Grand

$14 \mathrm{LB} 20$

Macedonia

Marble Rock

Do

Marshalltown

Do .

14RA2

10LA19

10RB13

9LB10

9LA9

$9 \mathrm{LB} 13$

11RB20

13RB14

9RB13

9LA11

9RB10

14LA22

13LA19
13LB16

14LB21

14LB22

9LB11

9LB12

9LB9

9LB14

13LA16

9RB11

9RB12

Mason City

Do........ 13LB13

Montour. .

Mt. Pleasant

14RB23

11RA19

Do........ 13LB20

Do....

Pella Station.

Marion $\mathrm{Co}$

Quarry, Marsball

Co.

Sigourney Station.

Keokuk Co.

Do.

14LA20

9RA10

14RB21

13LA21

9RA11

13RA20
Lt-drab, med-gr, compact, fossil, Carb.* Lt-buff, coarse-gr, compact, Carb.* Gray, med-gr, compact, semi-eryst. Sub-Carb.

Lt-gray, med-gr, compact, oolitic. Buff, fine-gr, compact, Devonian.* Lt-gray, coarse-gr, compact,

Devonian.*

Lt-gray, med-gr, compact, Sub-Carb.* Do.

Buff, med-gr, compact, fossil.*

Gray, fine-gr, compact, argil, mg,

Drab and buff-mottled, coarse-gr, fossil-bearing, Devonian.

Lt-gray, med-gr, compact, oolitic, L. Carb.*

Lt-buff, fine-gr, compact, siliceous, Carb.*

Drab, fine-gr, compact, cellular, Devonian.*

Lt-buff, coarse-gr, compact, fossil, Devonian.*

Drab, fine-gr, compact, Devonian.*

Dark, mottled, fine-gr, compact, $\mathrm{mg}$, L. Sil.*

Lt-buff, fine-gr, compact, oolitic, L. Carb.*

Gray, fine-gr, compact, oolitic, L. Carb.

Lt-buff, med-gr, compact, hitum, I. Carb.*

Grav, fine-gr, compact, fossil, Carb.*

Drab, coarse-gr, compact, fossil, Carb.

Buff, coarse-gr, compact, fossil, Carb.*

- Gray, fine-gr, compact, micaceous, I.

Lt-gray, fine-gr, compact, mg, Carb.*

Gray, med-gr, compact, ferrug, L.

Carb.*

Buff, fine-gr, compact, fossil, Devonian.* Devonian
Do.

Dark, uneven-gr, compact, siliceous, Devonian.

Brown, med-gr, compact, Devonian.* Gray, coarse-gr, porous, fossil, Devonian.*

Lt-yellow, fine-gr, compact, I. Carb.* Gray, fine-gr, compact, L. Carb.*

Lt-gray, fine-gr, compact, L. Carb.*

Gray, med-gr, compact, siliceous, L. Carb.*

Gray, med-gr, compact, oolitic, mg,

L. Carb.*
Buff, coarse-gr, compact, Carb.*

Gray, fine-gr, compact, Devonian.*

Lt-buff, fine-gr, compact, Devonian.*

Gray, med-gr, porous, oolitic.

Lt-drab, fine-gr, compact, arenaceous cale.

Lt-buff, fine-gr, compact, fossil, Devonian.*

Buff, fine-gr, compact, cellular, mg,

Devonian.*
Buff, med-gr, compact, oolitic, L. Carh. Do.

Drab, fine-gr, compact, pyritiferous, I. Carb.*

Drah, fine-gr, compact, siliceous, L. Carb.

Gray, med-gr, compact, L. Carb.*

Lt-gray, fine-gr, compact, L. Carb.*

Gray, med-gr, compact, fossil, L. Carb.**

Lt-buff, fine-gr, compact, dendr, L.

Carb.t
Lt-gray, finc-gr, compact, L. Carb.*

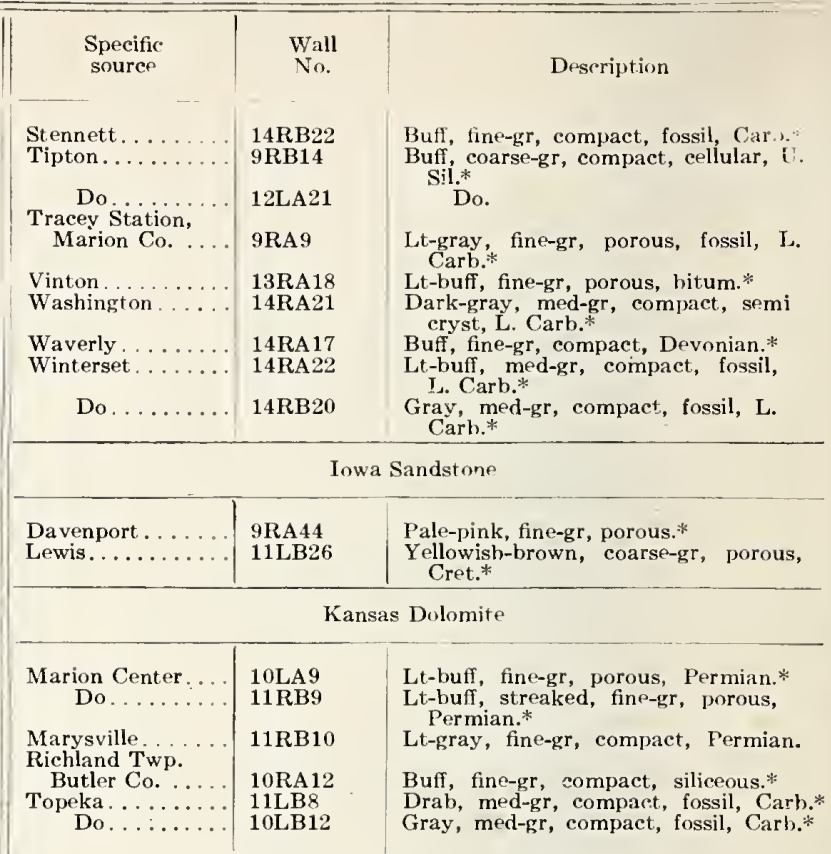

Kansas Limestone

See also $S-2,125$ of table 4

\begin{tabular}{l|l|l}
\hline Armstrong $\ldots \ldots \ldots$ & 10RA24 \\
Atchison....... & 11RA26 & $\begin{array}{l}\text { Buff, med-gr, porous, fossil.* } \\
\text { Dray-gray, med-gr, compact, fossil, } \\
\text { Carb. } * \\
\text { Buff, coarse-gr, porous, Permian }\end{array}$
\end{tabular}

Cottonwood ...... 11RA27

Fusulina.*

Lt-gray, coarse-gr, porous, Permian

Nearly white, porous, coarse-gr.

Cut from the same block as 11LB22.

Lt-gray, med-gr, porous, soft, fossil, Permian.*

Douglass........... 13RA24

Fildorado....... 10RA23

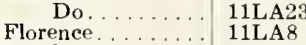

Greeley .......... 10LA25

Grenola ......... 10LA24

Irving

Junction City

14LB26

Leavenworth … 12LB22

Lane ............ 9RA13

Manbattan ........ 9RA13

Oswego .......... 12RB23

Rock Twp.

Butler Co. .... 12L B23

Silverdale.

$4 \mathrm{C}$

Smoky Hill

\begin{tabular}{l|l}
$\begin{array}{l}\text { Smoky Hiti } \\
\text { Reservation...... }\end{array}$ & 10RA22 \\
Wilson.......... & $12 \mathrm{RB} 22$
\end{tabular}

Winfield .......... 13LA25

Lt-gray, fine-gr, compact, Permian.*

Lt-gray, fine-gr, compact, fossii, mg.* Buff, coarse-grr, porous.*

Drab, fine-gr, compact, Fusulina.

Gray, fine-gr, compact, fossil.*

Buff, fine-gr, porous, soft and earthy.

Yellow, earthy, fine-gr, compact, mg.

fray, med-gr, compact, fossil.*

Lt-gray, fine-gr, compact, Permian.*

Dark-gray, compact, coarse-gr, fossil.*

Lt-drab, fine-gr, compact.

Buff, med-gr, compact calc,

White, fine-gr, porous, chalky.

Buff, fine-gr, compact.

Drah, fine-gr, compact, Permian.

Kansas Sandstone

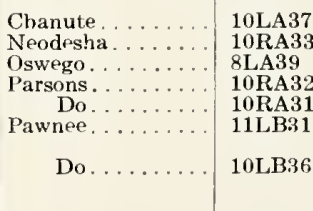

Buff, fine-gr, friable, calk.

Do.
Dark-gray, med-gr, porous, calk.*

Dark-gray, med-gr,

Gray, fine-gr, porous, calk.

Gray, dark-spotted, med-gr, porous, calk.*

Gray, dark-spotted, med-gr, compact,

Kentucky Dolomite
Bardstown

Lincoln Co.

Louisville.
11LA11

12LA13

$12 \mathrm{LB} 12$
Drah, fine-gr, compact, U. Sil

Lt-drab, fine-gr, compaet, semicryst.*
Gray, fine-gr, compact, fossil calc, I. 
TABLE 1. Source, classification, and description of domestic stones in south face of wall-Continued

\begin{tabular}{l|l|l|l|l|l|l|l}
$\begin{array}{l}\text { Specific } \\
\text { source }\end{array}$ & $\begin{array}{l}\text { Wall } \\
\text { No. }\end{array}$ & Description \\
\hline
\end{tabular}

\section{Kentucky Limestone}

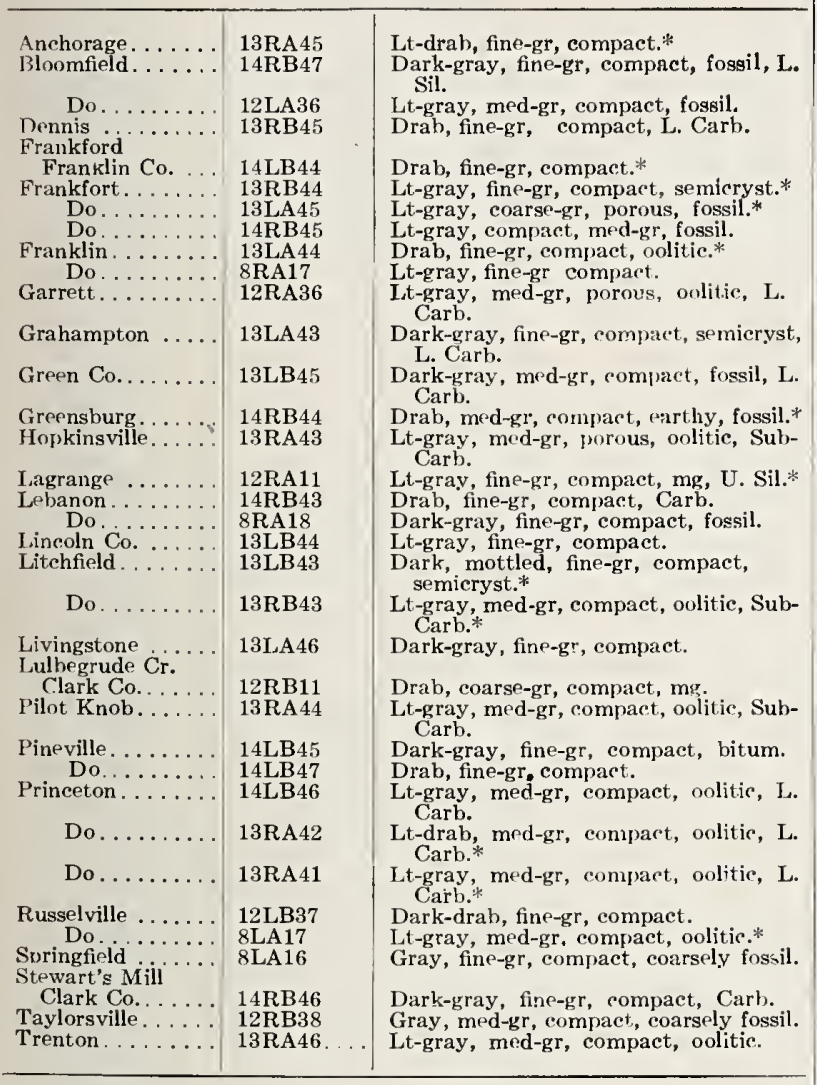

\section{Kentucky Sandstone}

\begin{tabular}{|c|c|c|}
\hline 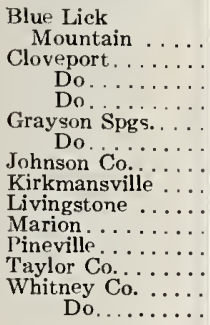 & $\begin{array}{l}\text { 8RB31 } \\
\text { 8LB31 } \\
\text { 7RA28 } \\
\text { 6LB30 } \\
\text { 5LA28 } \\
\text { 7LA28 } \\
\text { 8LA31 } \\
\text { 6RB29 } \\
\text { 6LA30 } \\
\text { 7RB27 } \\
\text { 8RA31 } \\
\text { 7LB28 } \\
\text { 6RA30 } \\
\text { 11LB45 }\end{array}$ & $\begin{array}{l}\text { Gray, fine-gr, compact. } \\
\text { Buff, fine-gr, porous, L. Carb. } \\
\text { Lt-brown, fine-gr, porous, L. Carb. } \\
\text { Buff, fine-gr, porous, L. Carb. } \\
\text { Buff, rust-spotted, fine-gr, porous. } \\
\text { Lt-gray, fine-gr, porous. } \\
\text { Lt-brown, med-gr, porous, L. Carb. } \\
\text { Yellowish, med-gr, porous.* } \\
\text { Gray, fine-gr, porous. } \\
\text { Nearly white, fine-gr, compact, I. Carb } \\
\text { Lt blue-gray, fine-gr, compact. } \\
\text { Gray, brown lines, fine-gr, compact. } \\
\text { Lt blue-gray, fine-gr, porous. } \\
\text { Lt-gray, fine-gr, porous. }\end{array}$ \\
\hline
\end{tabular}

Louisiana Quartzite

\begin{tabular}{l|l|l}
\hline Unknown. ...... & $6 \mathrm{LB} 47$ & Drab, white-dotted, fine-gr, compact. \\
\hline
\end{tabular}

\begin{tabular}{l|l|l}
\hline \multicolumn{3}{c}{ Louisiana Sandstone } \\
\hline Unknown ....... & $6 \mathrm{LA} 47$ & Pinkish, medium-gr, porous. \\
\hline
\end{tabular}

Maine Argillite (Slate)

\begin{tabular}{l|l|c}
\hline Brownville........ & 10RB26 \\
Monson......... & 10LB28 & $\begin{array}{c}\text { Uniform blue-black, electrical.* } \\
\text { Do. }\end{array}$ \\
\hline
\end{tabular}

\begin{tabular}{|c|c|c|}
\hline $\begin{array}{l}\text { Specific } \\
\text { source }\end{array}$ & $\begin{array}{l}\text { Wall } \\
\text { No. }\end{array}$ & Description \\
\hline \multicolumn{3}{|c|}{ Maine Diabase } \\
\hline $\begin{array}{l}\text { Addison } \\
\text { Addison Point. } \\
\text { Do....... } \\
\text { Vinal Haven . }\end{array}$ & $\begin{array}{l}\text { 5RB27 } \\
8 \mathrm{LA} 27 \\
\text { 5LB25 } \\
\text { 10LA30 }\end{array}$ & $\begin{array}{l}\text { Drak-gray, finc-gr, Devonian.* } \\
\text { Dark-gray, fine-gr, olivine.* } \\
\text { Do. } \\
\text { Do. }\end{array}$ \\
\hline \multicolumn{3}{|c|}{ Maine Gneiss } \\
\hline $\begin{array}{l}\text { Jefferson . ...... } \\
\text { Turner....... }\end{array}$ & $\begin{array}{l}\text { 7RB23 } \\
\text { 6RA25 }\end{array}$ & $\begin{array}{l}\text { Gray, fine-gr, muscovite-biotite.* } \\
\text { Dark-gray, med-gr, biotite.* }\end{array}$ \\
\hline
\end{tabular}

Maine Granite

See also $\mathrm{S}-1$ and $\mathrm{S}-2$ of table 4

\begin{tabular}{|c|c|c|}
\hline $\begin{array}{l}\text { Augusta } \\
\text { Biddeford } \\
\text { Do }\end{array}$ & $\begin{array}{l}\text { 7RB24 } \\
\text { 5RA27 } \\
\text { 5RA26 }\end{array}$ & $\begin{array}{l}\text { Itt-gray, fine-gr, muscovite-biotite. } \\
\text { Gray, coarse-gr, hiotite.* } \\
\text { Do. }\end{array}$ \\
\hline
\end{tabular}

Do......... 5RA2

Brunswick...... 6RA27

Bryant's Pond .... 6RB26

Calais............ 6 6RB25

Chesterville ........

Croteh Isl......... 7RA24

Dedham....... 8 RB27

Dix Island ...... 10LA32

E. Blue Hill ....... 10RB27

Franklin......... 6R B227

Do......... B16

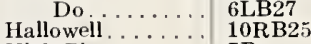

High Pine ........

Hurricane Isl. . . . 9RB23

Lincoln......... 7 LB25

Lincolnville ........ 6L 6 6 26

Milford .......... 4RB17

Mt. Desert. . . . . 12B

Do........ 10LB30

Nortb Jay ........ $5 \mathrm{~B}$

Otter Creek

\begin{tabular}{c|c} 
Mt. Desert. .... & 4LB17 \\
Pownal. ........
\end{tabular}

Round Pond ...... 7LA24

St. George $\ldots . .$.
Somesville

Mt. Desert. . . . 10RB28

Do.......... 10LA31

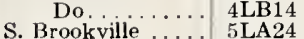

$\begin{array}{lll}\text { S. Brookville ...... } & \text { 5LA24 } \\ \text { St. Thomaston... } & 10 \mathrm{LB} 29\end{array}$

\begin{tabular}{r|l} 
St. Thomaston.... 10LB29 & 10LA34 \\
Do ........ & 10LA
\end{tabular}

\begin{tabular}{r|r} 
Do \\
Swanvilie............
\end{tabular}

Vinal Haven ....... 10LA33

\begin{tabular}{c|c|c} 
Do ............. & $7 \mathrm{LA25}$ \\
Do & 4RB15
\end{tabular}

\begin{tabular}{ll|l} 
Do............ & $4 \mathrm{RB} 15$ \\
Do ........ & $4 \mathrm{LB} 15$
\end{tabular}

Waldoborough.....

\begin{tabular}{ll|l} 
Wayne & ............ & 6LA25 \\
W. Sullivan &
\end{tabular}

Williamantic $\ldots . . .6$ 6LB25

Drak-gray, med-gr, biotite.

Pinkish-gray, med-gr, biotite.

Dark-gray, coarse-gr, biotite.

Gray, fine-gr, biotite.*

Mauve and white, coarse-gr, porphyritic biotite.*

Gray and white, coarse-gr, porphyritic biotite.*

Gray, coarse-gr, biotite.*

Do.

Do.

Gray, med-gr, biotite, "Mt. Waldo".*

Gray, coarse-gr, bintite.*

Lray, coarse-gr, bintite.*

Lt-gray, fine-gr, muscovite-biotite.*
Pink, coarse-gr, biotite, "Swenson

Gray, coarse-gr, biotite.*

Gray, coarse-gr, hornblende.*

Lt-gray, fine-gr, muscovite-biotite.*

Pink, coarse-gr, biotite, "Milford

Pink".*

Pink, medium to coarse, biotite,

"Somes Sound".

Pink, coarse-gr, biotite.*

Lt-gray, fine-gr, biotite-muscovite,

Bright-pink, coarse-gr, biotite.

Gray, fine-gr, biotite.

Dark-gray, fine-gr, biotite.*

Gray, med-gr, biotite.*

Lt-pink, coarse-gr, biotite.*

Pink and mauve, coarse-gr, biotite.*

Gray, coarse-gr, hiotite.*

Do.

Gray, fine-gr, biotite.*

Gray, fine-gr, biotite.*

Do.

Do.
Pink, coarse-gr, biotite.

Gray, fine-gr, biotite.*

Gray, coarse-gr, biotite.*

Gray, med-gr, biotite.*

Gray, coarse-gr, biotite.

Maine Schist

\begin{tabular}{l|l|l} 
Knightsville..... & $8 \mathrm{RA} 27$ & Dark-gray, fine-gr, compact, talcose**
\end{tabular}

Maine Serpentine

\begin{tabular}{l|l|l} 
Deer Island ..... & $5 \mathrm{LB} 24 \quad$ Black, compact.
\end{tabular}

Maryland Argillite (Slate)

\begin{tabular}{l|l|l}
\hline Nr. Cardiff...... & 6RA41 & Blue-black, med-coarse texture, roofing.*
\end{tabular} 
TABLE 1. Source, classification, and description of domestic stones in south face of wall-Continued

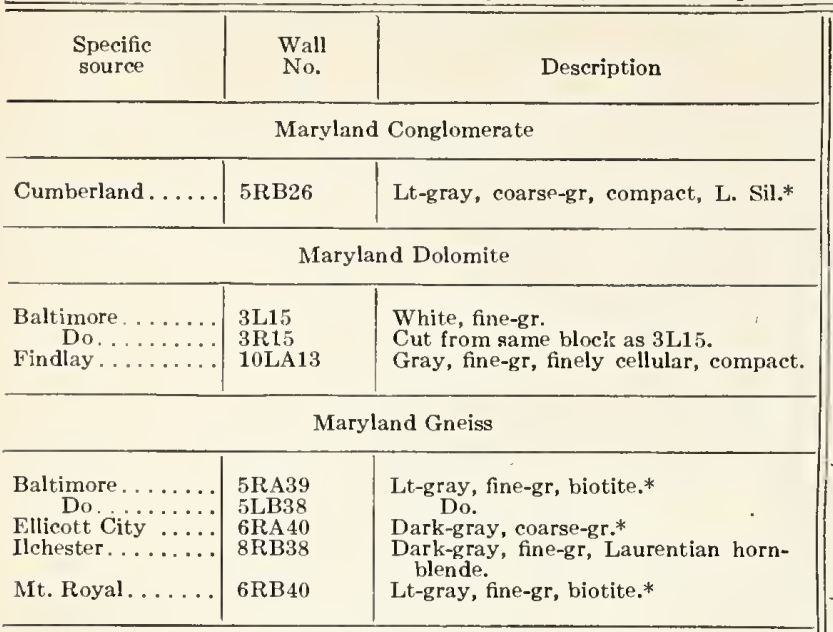

Maryland Granite

\begin{tabular}{|c|c|c|}
\hline 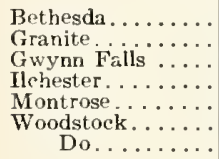 & $\begin{array}{l}5 \mathrm{LA} 38 \\
10 \mathrm{RB} 41 \\
10 \mathrm{RB} 40 \\
6 \mathrm{LA} 40 \\
5 \mathrm{RB} 39 \\
7 \mathrm{LB} 38 \\
6 \mathrm{LB} 40\end{array}$ & $\begin{array}{l}\text { Bluish-gray, med-gr, biotite.* } \\
\text { Black, white-spotted, coarse-gr, biotite. } \\
\text { Gray, fine-gr, biotite.* } \\
\text { Red, coarse-gr, Laurentian, biotite. } \\
\text { Gray, fine-gr, biotite.* } \\
\text { Gray, med-gr, biotitc.* } \\
\text { Do. }\end{array}$ \\
\hline
\end{tabular}

Maryland Limestone

\begin{tabular}{l|l|l}
\hline Mt. Pleasant. ... & 9 LB16 & Black, finc-gr, compact.* \\
\hline
\end{tabular}

Maryland Marble

See also S-2, 163 of table 4

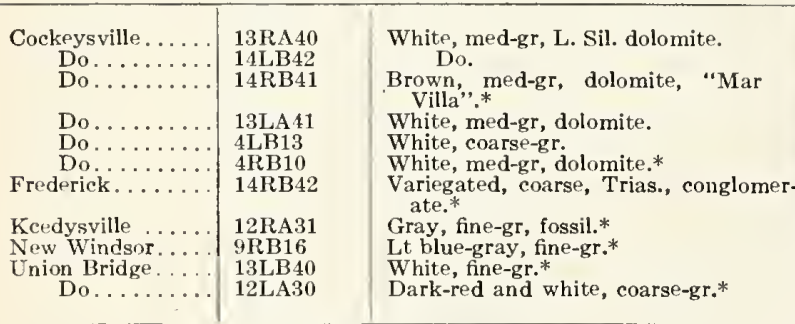

Maryland Quartzite

\begin{tabular}{l|l|l}
\hline Frederick Co...... & 10LB41 & Cream-colored, coarse-gr.* \\
\hline
\end{tabular}

Maryland Schist

See sections S-2 and EE of tahle 4

Maryland Serpentine

\begin{tabular}{|c|c|c|}
\hline 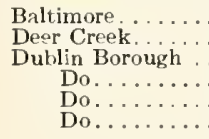 & $\begin{array}{l}\text { 7LA38 } \\
\text { 7LA37 } \\
\text { 7RB38 } \\
\text { 6RB41 } \\
\text { 8LB41 } \\
\text { 7RA38 }\end{array}$ & $\begin{array}{l}\text { Dark-green, fine-gr, compact. } \\
\text { Do. } \\
\text { Dark and light-green, variegated.* } \\
\text { Do. } \\
\text { Lt-green, variegated.* } \\
\text { Cut from same block as } 8 \mathrm{LB} 41 \text {. }\end{array}$ \\
\hline
\end{tabular}

Massachusetts Conglomerate

\begin{tabular}{l|l|l|}
\hline Dorchester...... & 7RB35 & $\begin{array}{l}\text { Vari-colored, compact, siliceous, } \\
\text { "Puddingstone".* }\end{array}$ \\
\hline \multicolumn{2}{|c|}{ Massachusetts Diabase } \\
\hline $\begin{array}{l}\text { E. Longmeadow ... } \\
\begin{array}{l}\text { Medford. ....... } \\
\text { SRB16 }\end{array}\end{array}$ & $\begin{array}{l}\text { Dark-gray, fine-gr, Mesozoic.* } \\
\text { Dark-gray, coarse-gr.* } \\
\text { Gray, coarse-gr.* }\end{array}$
\end{tabular}

\begin{tabular}{|c|c|c|}
\hline $\begin{array}{l}\text { Becket....... } \\
\text { Do..... } \\
\text { Charlton.... } \\
\text { Monson..... } \\
\text { Do. . . } \\
\text { Northfield... } \\
\text { W. Andover. } \\
\text { Westford..... } \\
\text { Do..... } \\
\text { Do..... } \\
\text { Do..... }\end{array}$ & $\begin{array}{l}10 \mathrm{LB} 23 \\
8 \mathrm{RA} 22 \\
9 \mathrm{LB} 24 \\
6 \mathrm{LA} 39 \\
10 \mathrm{LB} 22 \\
10 \mathrm{LB} 25 \\
6 \mathrm{LB} 22 \\
5 \mathrm{LB} 14 \\
5 \mathrm{LB} 15 \\
5 \mathrm{LB} 13 \\
7 \mathrm{LB} 37\end{array}$ & $\begin{array}{l}\text { Dark-gray, fine-gr, muscovite.* } \\
\text { Pink-gray, fine-gr, biotite-muscovite.* } \\
\text { Lt-gray, fine-gr, muscovite-biotite.* } \\
\text { Dark-gray, fine-gr, biotite.* } \\
\text { Gray, fine-gr, muscovite.* } \\
\text { Gray,, fine-gr, biotite.* } \\
\text { Gray, coarse-gr, muscovite.* } \\
\text { Gray, fine-gr, muscovite.* } \\
\text { Lt-gray, fine-gr, muscovite.* } \\
\text { Gray, fine-gr, muscovite.*. } \\
\text { Lt-gray, fine-gr, muscovite.* }\end{array}$ \\
\hline
\end{tabular}

Massachusetts Granite

See also $\mathrm{S}-1$ and $\mathrm{S}-2$ of table 4

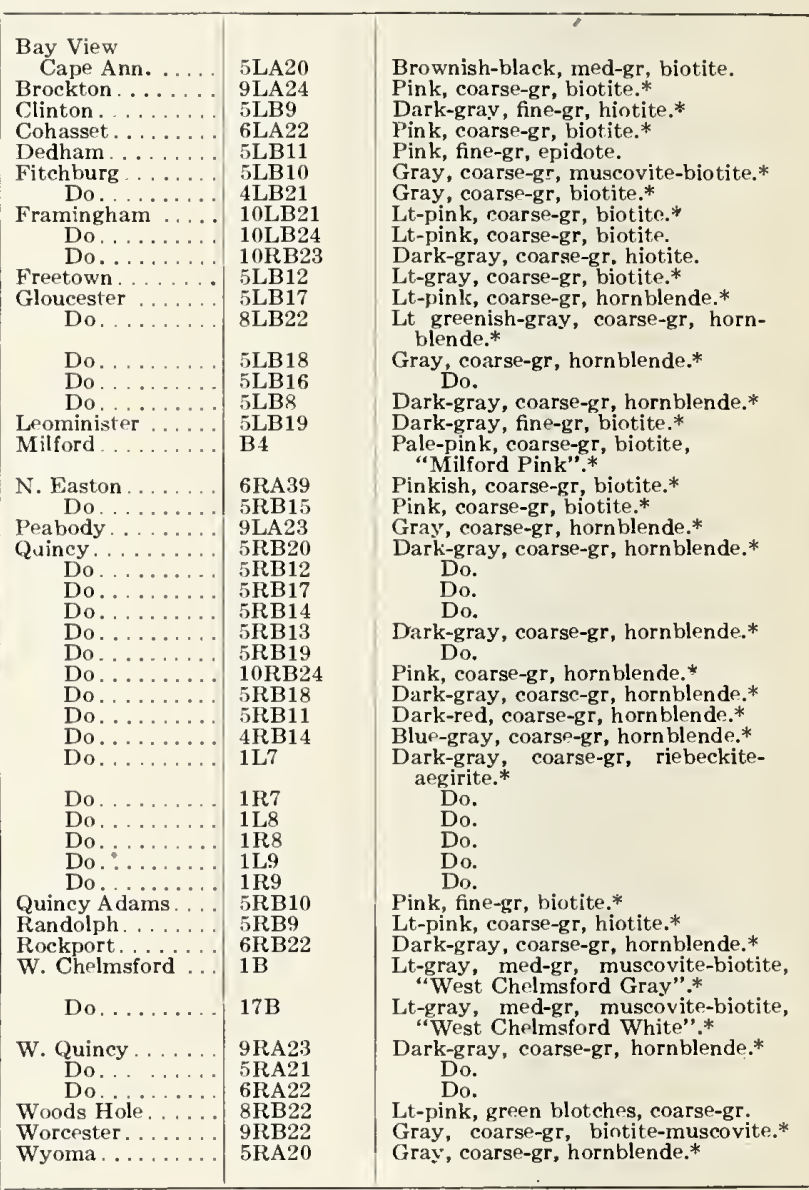

Massachusetts Marble

See also S-2, 164 to 166 of table 4

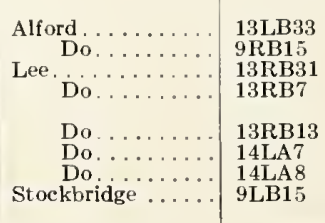

White, med-gr, L. Sil.

Grayish-white, coarse-gr, L. Sil.* Wrayish-white, coarse-gr, L.. St.* White, med-gr, dolomite, "Lee White, med-gr,
Marble"*

Do.

Do.

Wa ter-blue, fine-gr, L. Sil.* 
TABLE 1. Source, classification, and description of domestic stones in south face of wall-Continued

\begin{tabular}{|c|c|c|}
\hline $\begin{array}{l}\text { Specific } \\
\text { source }\end{array}$ & $\begin{array}{l}\text { Wall } \\
\text { No. }\end{array}$ & Description \\
\hline \multicolumn{3}{|c|}{ Massachusetts Melaphyre } \\
\hline Brighton......... & 10LB26 & $\begin{array}{l}\text { Olive-green, whit } \rho \text {-speckled, med-gr, } \\
\text { compact.* }\end{array}$ \\
\hline \multicolumn{3}{|c|}{ Massachusetts Sandstone } \\
\hline $\begin{array}{l}\text { E. Long Meadow } \\
\text { Do......... }\end{array}$ & $\begin{array}{l}\text { 8RA23 } \\
9 \mathrm{RQ}\end{array}$ & $\begin{array}{l}\text { Lt reddish-brown, porous, fine-gr." } \\
\text { Reddish-brown, ,fnc-gr, Trias. } \\
\text { "Longmeadow".* }\end{array}$ \\
\hline \multicolumn{3}{|c|}{ Miehigan Argillite (Slate) } \\
\hline Arvon.... & $6 \mathrm{LA} 45$ & Blue-black, smooth texture slate.* \\
\hline
\end{tabular}

Michigan Dolomite

\begin{tabular}{c|l|l}
\hline Raisinville ...... & 11LA2 & $\begin{array}{c}\text { Lt-gray, fine-gr, porous, granular, } \\
\text { Devonian.* } \\
\text { Drab, fine-gr, compact, fossil. } \\
\text { Devonian.* }\end{array}$ \\
\hline
\end{tabular}

Michigan Gneiss

\begin{tabular}{l|l|l}
\hline Vulcan........ & 6 RA46 & Dark-gray, fine-gr, biotite.* \\
\hline
\end{tabular}

Michigan Granite

\begin{tabular}{l|l|l}
\hline Vulcan. . . . . . & 11RB39 & Red, fine-gr, biotite.*
\end{tabular}

Michigan Quartzite

\begin{tabular}{l|l|l}
\hline Vulcan........ & 10LA 49 & Lt-gray, fine-gr, compact, L. Sil.*
\end{tabular}

Michigan Sandstone

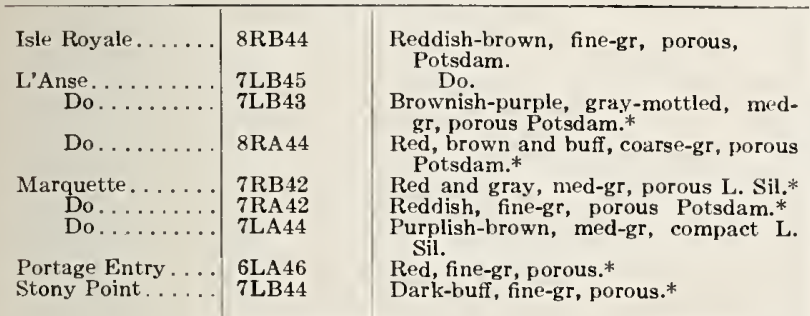

Minnesota Diabase

\begin{tabular}{l|l|l}
\hline Duluth. & Dark-gray, fine-gr, trap rock.* \\
Taylor's Falls .... & 5LA33 & 5RB33 \\
Two Harbor Bay.. & 6LB35 & Nearly biack, fine-gr, olivine** \\
& Nearly black, fine-gr.
\end{tabular}

Minnesota Dolomite

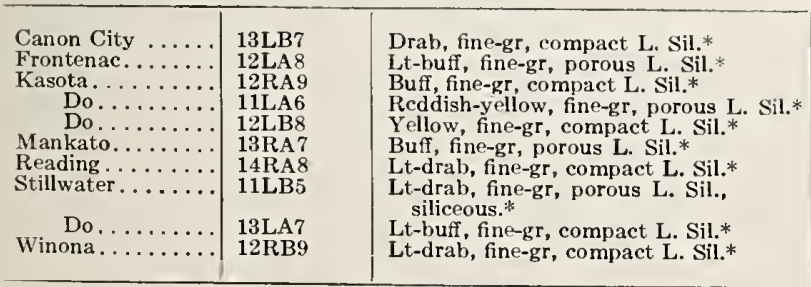

Minnesota Gabbro

\begin{tabular}{l|l|l}
\hline Duluth........ & 5LB34 & Dark-gray, coarse-gr, gabbro.* \\
\hline
\end{tabular}

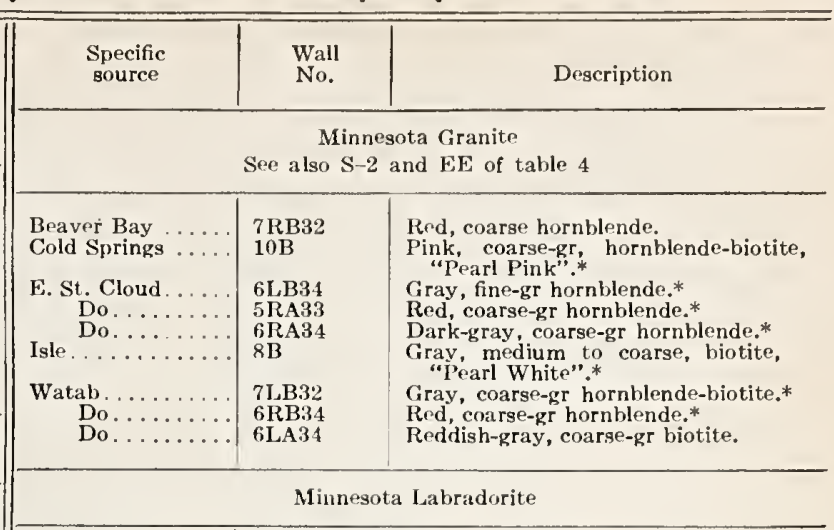

\begin{tabular}{l|l|l}
\hline Beaver City ..... & 6LA35 & Lt-greenish, coarge-gr, compact.
\end{tabular}

Minnesota Limestone

See also S-2 and $25 \mathrm{EE}$ of table 4

\begin{tabular}{|c|c|c|}
\hline Canon City & 13LB32 & Drab, fine-gr, compact L. Sil.* \\
\hline Mankato. & & Buff, fine-gr, cellular mg.* \\
\hline & $17 \mathrm{C}$ & $\begin{array}{l}\text { Gray-buff, fine-gr, cellular mg.* } \\
\text { Cream, fine-gr, cellular mg.* }\end{array}$ \\
\hline Minneapolis. & $14 \mathrm{RB} 8$ & Dark-mottled gray, finc-gr, compact, \\
\hline $\begin{array}{l}\text { Do... } \\
\text { Red Wing. }\end{array}$ & $\begin{array}{l}\text { 9LA16 } \\
\text { 11LA5 }\end{array}$ & $\begin{array}{l}\text { Mottled gray, fine-gr, compact.* } \\
\text { It-drab, fine-gr, compact, mg, }\end{array}$ \\
\hline St. Paul. & 9RA16 & $\begin{array}{l}\text { Gray, fine-gr, compact, fossil, } \mathrm{mg}, \mathrm{L} \text {. } \\
\text { Sil.* }\end{array}$ \\
\hline
\end{tabular}

Minnesota Quartz

\begin{tabular}{l|l|l}
\hline Duluth....... & 10LA35 & $\begin{array}{l}\text { Reddish-brown, fine-gr, compact } \\
\text { porphyry. } \\
\text { Lull-red, fine-gr, compact porphyry. }\end{array}$ \\
\hline
\end{tabular}

Minnesota Quartzite

\begin{tabular}{l|l|l} 
Courtland....... & 5LB33 & Purplish-red, fine-gr, compact.*
\end{tabular}

\begin{tabular}{|c|c|c|}
\hline \multicolumn{3}{|c|}{ Minnesota Sandstone } \\
\hline $\begin{array}{l}\text { Dakota. .... } \\
\text { Dresback ... } \\
\text { Fond Du Lac } \\
\text { Hinckley..... } \\
\text { Jordan..... }\end{array}$ & $\begin{array}{l}\text { 8RB34 } \\
\text { 7RA32 } \\
\text { 8LA34 } \\
\text { 7LA32 } \\
\text { 9RA33 }\end{array}$ & $\begin{array}{l}\text { Buff, fine-gr, friable L. Sil.* } \\
\text { Gray, fine-gr, friable L. Sil. } \\
\text { Purplish-brown fine-gr, compact L. Sil.* } \\
\text { Yellowish-pink, fine-gr, porous.* } \\
\text { Buff, rust-spotted, fine-gr, porous L. }\end{array}$ \\
\hline $\begin{array}{l}\text { Luverne .... } \\
\text { Mendota... }\end{array}$ & $\begin{array}{l}\text { 8RA34 } \\
8 \mathrm{LB} 34\end{array}$ & $\begin{array}{l}\text { Purplish-brown, med-gr, compact. } \\
\text { Bright-yellow, med-gr, porous L. Sil.* }\end{array}$ \\
\hline \multicolumn{3}{|c|}{ Mississippi Limestone } \\
\hline $\begin{array}{l}\text { Teshomingo } \\
\text { County... }\end{array}$ & $8 \mathrm{LB} 16$ & Gray, fine-gr, compact, finely fossil. \\
\hline \multicolumn{3}{|c|}{ Mississippi Sandstone } \\
\hline
\end{tabular}

Rankin Co.

\begin{tabular}{l|l}
8 LA11 & Yellow, fine-gr, compact argil.
\end{tabular}

Missouri Dolomite

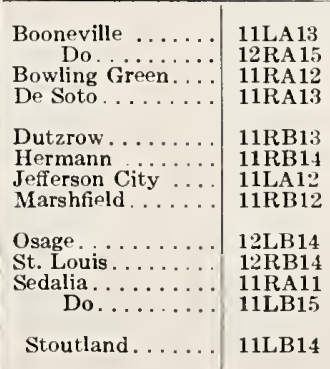

Buff, fine-gr, compact.*

Mottled-buff, fine-gr, compact L. Sil.* Lt-gray, fine-gr, compact, siliceous $\mathbf{L}$. Sil.**
Lt-drab, fine-gr, compact.*

Lt-drab, fine-gr, compact.*
Dark-gray, fine-gr, compact siliceous.* Lt-gray, fine-gr, compact siliceous.* Yellow-brown, fine-gr, compact siliceous.*

Lt-spotted, coarse-gr, porous siliceous.* Lt-gray, fine-gr, compact L. Carb.* Buff, fine-gr, compact fossil.*

Dark-gray, fine-gr, compact siliceous

Lt-gray, fine-gr, siliceous.* 
TABLE 1. Source, classification, and description of domestic stones in south face of wall-Continued

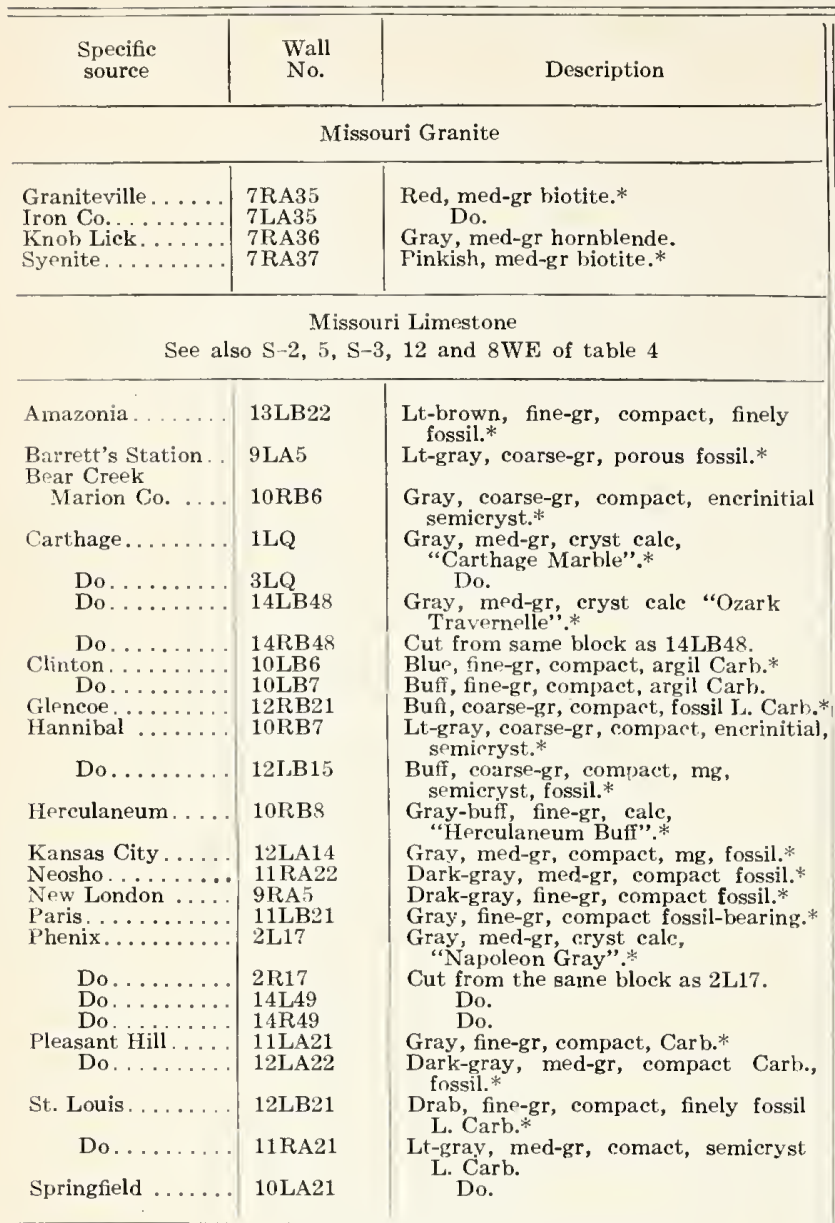

Missouri Marble

See also S-2, 141 of table 4

\begin{tabular}{l|l|l}
\hline Cape Girardeau ... & 11RB21 & Nearly white, med-gr, L. Sil.* \\
\hline
\end{tabular}

Missouri Sandstone

\begin{tabular}{|c|c|c|}
\hline Butler. & 11LB30 & Gray, rust-spotted, med-gr, porous \\
\hline $\begin{array}{r}\text { Clinton..... } \\
\text { Do }\end{array}$ & $\begin{array}{l}\text { 8LA36 } \\
8 \mathrm{RB} 37\end{array}$ & $\begin{array}{l}\text { Lt-gray, fine-gr, porous Carb.* } \\
\text { Buft, fine-gr, compact Carb.** }\end{array}$ \\
\hline Howard Co... & 8RA37 & Buff, fine-gr, porous Carb. \\
\hline Ralls Co. & 11LB29 & Lt-buff, fine-gr, porous.* \\
\hline $\begin{array}{l}\text { Meadville. } \\
\text { Miami Station }\end{array}$ & 9RB32 & Buff, med-gr, porous, calk Carb.* \\
\hline $\begin{array}{l}\text { Carroll Co... } \\
\text { Nevada....... }\end{array}$ & $\begin{array}{l}\text { 13RA22 } \\
8 \mathrm{RA} 36\end{array}$ & $\begin{array}{l}\text { Gray, fine-gr, porous Carb.* } \\
\text { Lt-gray, rust-spotted, fine-gr, porous, } \\
\text { Carb.* }\end{array}$ \\
\hline $\begin{array}{l}\text { Rockville...... } \\
\text { Do...... } \\
\text { St. Genevieve } \\
\text { Warrensburg. }\end{array}$ & $\begin{array}{l}\text { 8LB37 } \\
\text { 9RA34 } \\
\text { 10RB36 } \\
\text { 8LA37 }\end{array}$ & $\begin{array}{l}\text { Lt-buff, fine-gr, porous, calk, Carb.* } \\
\text { Red, coarse-gr, porous, calk Carb.* } \\
\text { Buf, fine-gr, porous I. Carb.* } \\
\text { Gray, med-gr, porous Carb.** }\end{array}$ \\
\hline
\end{tabular}

Montana Diorite

\begin{tabular}{l|l|l|}
\hline Helena ......... & 7RA40.... & Gray, med-gr.
\end{tabular}

Montana Granite

\begin{tabular}{|c|c|c|}
\hline $\begin{array}{l}\text { Baxendale } \ldots \ldots \ldots \\
\text { Butte.......... }\end{array}$ & $\begin{array}{l}\text { 10LB } 42 \\
10 \mathrm{RB} 43\end{array}$ & $\begin{array}{l}\text { Pink and gray, meä-gr biotite. } \\
\text { Dark greenish-gray, med-gr horn- }\end{array}$ \\
\hline Lewis \& Clark Co. & $7 \mathrm{LB} 41$ & $\begin{array}{l}\text { blende. } \\
\text { Gray, med-gr porphyritic biotite. }\end{array}$ \\
\hline
\end{tabular}

\begin{tabular}{|c|c|c|}
\hline $\begin{array}{l}\text { Specific } \\
\text { source }\end{array}$ & $\begin{array}{l}\text { Wall } \\
\text { No. }\end{array}$ & Description \\
\hline \multicolumn{3}{|c|}{$\begin{array}{l}\text { Montana Marble } \\
\text { See also S-2, 133, and } 134 \text { of table } 4\end{array}$} \\
\hline $\begin{array}{r}\text { Helena . . . . . } \\
\text { Do. . }\end{array}$ & $\begin{array}{l}\text { 11LA32 } \\
12 \mathrm{LB} 13\end{array}$ & $\begin{array}{l}\text { Black and gold, fine-gr. } \\
\text { Dark-gray, fine-gr, brecciated } \\
\text { dolomite. }\end{array}$ \\
\hline \multicolumn{3}{|c|}{ Montana Sandstone } \\
\hline 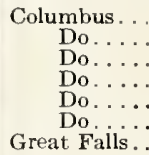 & $\begin{array}{l}\text { 7LA41 } \\
\text { 8RB43 } \\
\text { 8LB43 } \\
\text { 8RA43 } \\
\text { 8LA43 } \\
\text { 8RB42 } \\
\text { 11RB35 }\end{array}$ & $\begin{array}{l}\text { Dray-gray, fine-gr, compact, Cret. } \\
\text { Do. } \\
\text { Do. } \\
\text { Do. } \\
\text { Dark-gray, fine-gr, porous, Cret. } \\
\text { Do. } \\
\text { Brownish-purple, fine-gr, compact. }\end{array}$ \\
\hline
\end{tabular}

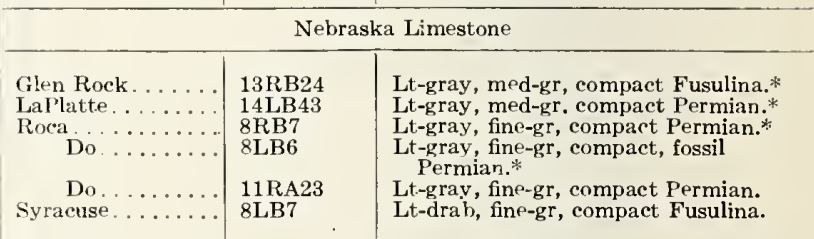

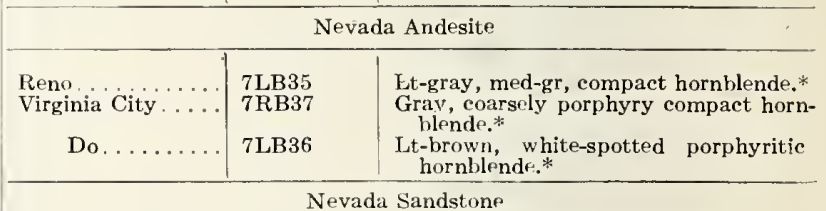

\begin{tabular}{l|l|l}
\hline Carson City ...... & 7RB36 & Gray, coarse-gr porous.*
\end{tabular}

\begin{tabular}{l|l|l}
\hline \multicolumn{3}{|c}{ New Hampshire Breccia } \\
\hline Franconia........ & 11LB34 & $\begin{array}{l}\text { Red, compact breccia of porphyry } \\
\text { fragments. }\end{array}$ \\
\hline \multicolumn{2}{|c}{ New Hampshire Gneiss } \\
\hline $\begin{array}{l}\text { Petersborough .... } \\
\text { Sunapee......... }\end{array}$ & 6RA43 & $\begin{array}{l}\text { Gray, med-gr muscovite-biotite.* } \\
\text { Dark-gray, med-gr biotite.* }\end{array}$ \\
\hline
\end{tabular}

New Hampshire Granite

See also $\mathrm{S}-2,4 \AA, 76$, and 80 of table 4

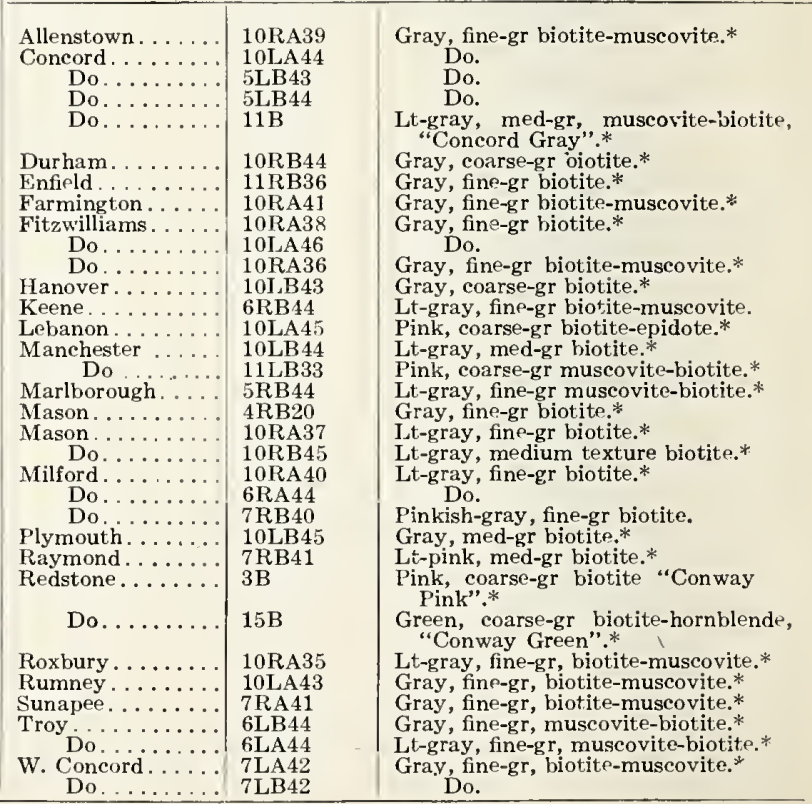


TABLE 1. Source, classification, and description of domestic stones in south face of wall Continued

\begin{tabular}{|c|c|c|}
\hline $\begin{array}{l}\text { Specific } \\
\text { source }\end{array}$ & $\begin{array}{l}\text { Wall } \\
\text { No. }\end{array}$ & Description \\
\hline \multicolumn{3}{|c|}{ New Jersey Conglomerate } \\
\hline $\begin{array}{l}\text { Boonton ...... } \\
\text { May's Landing } \\
\text { Atlantic Co.. } \\
\text { Morristown ... }\end{array}$ & $\begin{array}{l}\text { 5RB43 . } \\
\text { 9́LA36 } \\
\text { 5LB42 }\end{array}$ & $\begin{array}{l}\text { Reddish, coarse-gr, compact. } \\
\text { Brown, coarse-gr, porous, ferrug. } \\
\text { Reddish-brown and white, coarse-gr, } \\
\text { compact. }\end{array}$ \\
\hline \multicolumn{3}{|c|}{ New Jersey Diabase } \\
\hline $\begin{array}{l}\text { Jersey City.... } \\
\text { Do..... } \\
\text { Lambertville . } \\
\text { Rock Church.. } \\
\text { Rocky Hill... } \\
\text { Weehawken... } \\
\text { W. New York }\end{array}$ & $\begin{array}{l}\text { 10LA42 } \\
\text { 10RB46 } \\
6 \mathrm{LB} 43 \\
\text { 5LA42 } \\
6 \mathrm{RB} 43 \\
\text { 5RA43 } \\
\text { 10RA44 }\end{array}$ & $\begin{array}{l}\text { Dark-gray, fine-gr.* } \\
\text { Nearly biack, fine-gr.* } \\
\text { Dark-gray, fine-gr.* } \\
\text { Gray, fine-gr.* } \\
\text { Dark-gray, very fine-gr.* } \\
\text { Dark-gray, fine-gr.** } \\
\text { Gray, fine-gr.* }\end{array}$ \\
\hline
\end{tabular}

\section{New Jersey Dolomite}

\begin{tabular}{l|l|l}
\hline Newton........ & 13 LB12 & Dark-gray, fine-gr, compart, L. Sil.*
\end{tabular}

\begin{tabular}{l|l|l}
\hline \multicolumn{3}{c}{ New Jersey Gneiss } \\
\hline $\begin{array}{l}\text { Bloomingdale..... } \\
\text { Dover.......... }\end{array}$ & $\begin{array}{l}\text { 5RB42 } \\
\text { 5RA42 }\end{array}$ & $\begin{array}{l}\text { Greenish-gray, fine-gr, biotite.* } \\
\text { Greenish-gray, med-gr, non micaceous } \\
\text { hornblende.* }\end{array}$ \\
\hline \multicolumn{2}{c}{ New Jersey Granite }
\end{tabular}

\begin{tabular}{|c|c|c|}
\hline $\begin{array}{l}\text { Charlotteburgh ... } \\
\text { Pompton Jet..... }\end{array}$ & $\begin{array}{l}\text { 9LA35 } \\
\text { 5RA17 }\end{array}$ & $\begin{array}{l}\text { Pink, coarse-gr, biotite.* } \\
\text { Pink and green, coarse-gr, pegmatite, }\end{array}$ \\
\hline$\underset{\text { Morristown }}{\text { Do } \ldots \ldots}$ & $\begin{array}{l}\text { 5LB41 } \\
\text { 5LA41 }\end{array}$ & $\begin{array}{l}\text { Do. } \\
\text { Dark-green and white coarse-gr, horn- } \\
\text { blende. }\end{array}$ \\
\hline
\end{tabular}

\begin{tabular}{|c|c|c|}
\hline \multicolumn{3}{|c|}{ New Jersey Sandstone } \\
\hline Avondale. & 7RB39 & $\begin{array}{l}\text { Brownish-purple, med-gr, porous } \\
\text { Trias* }\end{array}$ \\
\hline Belleville. & $8 \mathrm{RB} 40$ & Do. \\
\hline Do.. & 8RA40 & Brown, med-gr, porous Trias.* \\
\hline Do. & 9RB36 & $\begin{array}{l}\text { Brownish-purple, fine-gr, porous } \\
\text { Trias.* }\end{array}$ \\
\hline Do. & 7RA39. & $\begin{array}{l}\text { Brownish-purple, med-gr, porous } \\
\text { Trias.* }\end{array}$ \\
\hline $\begin{array}{l}\text { Danville... } \\
\text { Greensburg. }\end{array}$ & $\begin{array}{l}\text { 8RB41 } \\
\text { 8RA41 }\end{array}$ & $\begin{array}{l}\text { Dark-gray, coarse-gr, compact.* } \\
\text { Lt brownish-purple, coarse-gr, com- }\end{array}$ \\
\hline $\begin{array}{l}\text { I.tittle Falls. } \\
\text { Martinville.. } \\
\text { Newark ..... } \\
\text { Do..... }\end{array}$ & $\begin{array}{l}\text { 8RB39 } \\
\text { 6RA442 } \\
\text { 8LA42 } \\
\text { 7LB40 }\end{array}$ & $\begin{array}{l}\text { Lt-brown, fine-gr, porous Trias.* } \\
\text { Drab, fine-gr, compact Trias.* } \\
\text { Purplish-brown, fine-gr, porous Trias.* } \\
\text { Brownish-purple, fine-gr, porous } \\
\text { Trias.* }\end{array}$ \\
\hline $\begin{array}{l}\text { Orange Mt. . } \\
\text { Patterson... }\end{array}$ & $\begin{array}{l}\text { 7LA40 } \\
\text { 7LA399 }\end{array}$ & $\begin{array}{l}\text { Purplish-brown, med-gr, porous Trias. } \\
\text { Brownish-purple, med-gr, porous Trias. }\end{array}$ \\
\hline Pleasant Val. & 7LB39 & Brown, fine-gr, porous Trias.* \\
\hline $\begin{array}{l}\text { Do...... } \\
\text { Princetown.. }\end{array}$ & $\begin{array}{l}\text { 6LA43 } \\
11 \mathrm{RB} 38\end{array}$ & $\begin{array}{l}\text { Brown, fine-gr, porous 'Trias. } \\
\text { Greenish-gray, fine-gr, compact Trias.* }\end{array}$ \\
\hline & $8 \mathrm{LB} 42$ & $\begin{array}{l}\text { Dark hlue-gray, fine-gr, compart } \mathrm{L} \text {. } \\
\text { Sil.* }\end{array}$ \\
\hline Stockton. . & 6LA42 & Lt purplish-brown, coarse-gr, porous \\
\hline Do. & 6LB42 & $\begin{array}{l}\text { Lt-gray, rust-spotted, med-gr, com- } \\
\text { pact Trias.* }\end{array}$ \\
\hline Woodsville. & 11RB37 & Blue-black, fine-gr, compact.* \\
\hline
\end{tabular}

\begin{tabular}{|c|c|c|}
\hline \multicolumn{3}{|c|}{ New Mexico Sandstone } \\
\hline $\begin{aligned} \text { Las } & \text { Vegas } \ldots . . . . \\
& \text { Do } \ldots \ldots \ldots \ldots\end{aligned}$ & $\begin{array}{l}8 \mathrm{RA} 47 \\
11 \mathrm{RB} 45\end{array}$ & $\begin{array}{l}\text { Brownish-purple fine-gr, compact. } \\
\text { Lt-red and white-banded, med-gr, }\end{array}$ \\
\hline $\begin{array}{l}\text { Do...... } \\
\text { Do...... } \\
\text { Do.... } \\
\text { Santa Fe } \\
\text { Valencia Co. }\end{array}$ & $\begin{array}{l}\text { 7RA44 } \\
8 \mathrm{LA47} \\
11 \mathrm{LB} 44 \\
7 \mathrm{LA} 45 \\
8 \mathrm{LB} 47\end{array}$ & $\begin{array}{l}\text { Lorous. } \\
\text { Pt-gray, fine-gr, porous. } \\
\text { Pink, fine-gr, porous sandstone. } \\
\text { Purplish-red, fine-gr, compact. } \\
\text { Brownish-purple, coarse-gr, porous.* } \\
\text { Reddish-brown, fine-gr, porous.* }\end{array}$ \\
\hline
\end{tabular}

\begin{tabular}{|c|c|c|}
\hline \multicolumn{3}{|c|}{ New Mexico Serpentine } \\
\hline $\begin{array}{l}\text { Grant County } \\
\text { Gila River }\end{array}$ & 7RA45 & Green and white-banded, fine-gr, \\
\hline $\begin{array}{l}\text { Do...... } \\
\text { Do..... } \\
\text { Do.... }\end{array}$ & $\begin{array}{l}\text { 10LB49 } \\
\text { 10RB50 } \\
\text { 11RB43 }\end{array}$ & $\begin{array}{l}\text { Do. } \\
\text { Cut from same block as 10LB49. } \\
\text { Green and white-banded, fine-gr, } \\
\text { "Riccolite".* }\end{array}$ \\
\hline Do.... & 11RB44 & Cut from same block as 11RB43. \\
\hline
\end{tabular}

\begin{tabular}{|c|c|c|}
\hline $\begin{array}{l}\text { Specific } \\
\text { source }\end{array}$ & $\begin{array}{l}\text { Wall } \\
\text { No. }\end{array}$ & Description \\
\hline \multicolumn{3}{|c|}{ New Mexico Tuff } \\
\hline Santa Fe.... & $7 \mathrm{RB} 45$ & $\begin{array}{l}\text { Lt-gray, fine-gr, rhyolite tuff. } * \text { (used } \\
\text { in Santa Fe Cathedral) }\end{array}$ \\
\hline \multicolumn{3}{|c|}{ New York Argillite (Slate) } \\
\hline $\begin{array}{l}\text { Granville. . . . . . . } \\
\text { Hampton . . } \\
\text { Middle Granville. }\end{array}$ & $\begin{array}{l}\text { 9LA31 } \\
\text { 9RA30 } \\
\text { 10RB30 }\end{array}$ & $\begin{array}{l}\text { Green and red, rough texture, roofing.* } \\
\text { Green, rough-textarc, roofing slate. } \\
\text { Purple, rough-texture, roofing slate. }\end{array}$ \\
\hline
\end{tabular}

New York Dolomite

\begin{tabular}{l|l|l}
\hline $\begin{array}{l}\text { Lockport......... } \\
\text { Rochester....... }\end{array}$ & 13LB6 & $\begin{array}{l}\text { Lt-gray, fine-gr, compact, f } n \text { ssil } \& . \text { Sil.* } \\
\text { Dark-drab, fine-gr, compact Devonian.* }\end{array}$
\end{tabular}

New York Gneiss

\begin{tabular}{c|c|c|c|}
\hline Hastings......... & 6 LB29 & $\begin{array}{c}\text { Dark-gray, white-banded, fine-gr } \\
\text { biotite.* }\end{array}$ \\
\hline
\end{tabular}

New York Limestone

\begin{tabular}{|c|c|c|}
\hline Amsterdam .. & $14 \mathrm{RB} 6$ & $\begin{array}{l}\text { Nearly black, fine-gr, compact, } \mathrm{mg}, \mathrm{L} \text {. } \\
\text { Sil }\end{array}$ \\
\hline Buffalo. & 11RA6 & Dark-gray, med-gr, compart, mg, \\
\hline Canajoharie. & 12LA7 & Dark-gray, coarse-gr, compact, mg, \\
\hline Cobleskill & 12RA 8 & Black, fine-gr, compact, $\mathrm{mg}$ \\
\hline $\begin{array}{l}\text { Cotton wood Sta.. } \\
\text { Crown Point } \ldots\end{array}$ & $\begin{array}{l}\text { 13LA37 } \\
12 \mathrm{RA} 7\end{array}$ & $\begin{array}{l}\text { I.t-gray, med-gr, somewhat porous. } \\
\text { Nearly black, fine-gr, compact, mg, } \\
\text { U. Sil.* }\end{array}$ \\
\hline $\begin{array}{l}\text { Howe's Cave. } \\
\text { Do. . . } \\
\text { Do. . }\end{array}$ & $\begin{array}{l}13 \mathrm{RA} 5 \\
11 \mathrm{RB} 5 \\
14 \mathrm{RB} 7\end{array}$ & $\begin{array}{l}\text { Black, fine-gr, compact, mg, U. Sil.* } \\
\text { Black, fine-gr, compact, mg, U. Sil.* } \\
\text { Nearly black, fine-gr, compact, mg, } \\
\text { fossil U. Sil.** }\end{array}$ \\
\hline
\end{tabular}

Indian Res. \begin{tabular}{c|c}
$\begin{array}{c}\text { Indian Res. } \\
\text { Onondaga Co. . . }\end{array}$ & 12RB7 \\
Kingston......... & 13RB5
\end{tabular} $\begin{aligned} & \text { Gray, med-gr, compact, fossil, me.* } \\
& \text { Dark-gray, med-gr, compact, mg Carh. }\end{aligned}$

Le Roy........... 12LB7

Louisville....... 8LA6

Onondaga........ $10 \mathrm{LA} 7$

Prescott........ 10RA10

Sandy Hill ...... 8RA6

Saratoga........ 14RA7

Schoharie ......... 12LB6

S. Glenn Falls .... 14LB8

Springfield Center. 12RB8

Three Mile Bay . . 11RB7

Union Springs .... 11LA4

Waterloo........ 13LA6

Williamsville ..... 13RB35

Willsborough..... 13RB6

Dark-gray, med-gr, compact, mg Carh."

Dark-gray, fine-gr, compact, $\mathrm{mg}$
Devonian.*

Gray, med-gr, compact.*

Dark-gray, med-gr, compart, fossi!

Gray, med-gr, compact, fossil, mg L.

Dark-gray, fine-gr, compart L. Sil.*

Black, fine-gr, compact L. sil.

Black, fine-gr, compact, mg U. Sil.*

Nearly black, fine-gr, enmpant, ing L. Sil.*

Dark-drab, fine-gr, compact, $\mathrm{mg}$

Gray, fine-gr, compact, fossil, mp I.

Black, fine-gr, compaet, $\mathrm{mg}$

Devonian.

Black, fine-gr, compact., mg Devonian. Gray, med-gr, compact, fossii,
Devonian.*

Black, fine-gr, compact, fossil, mg $\mathrm{I}$.

New York Marble

See also S-2 of table 4

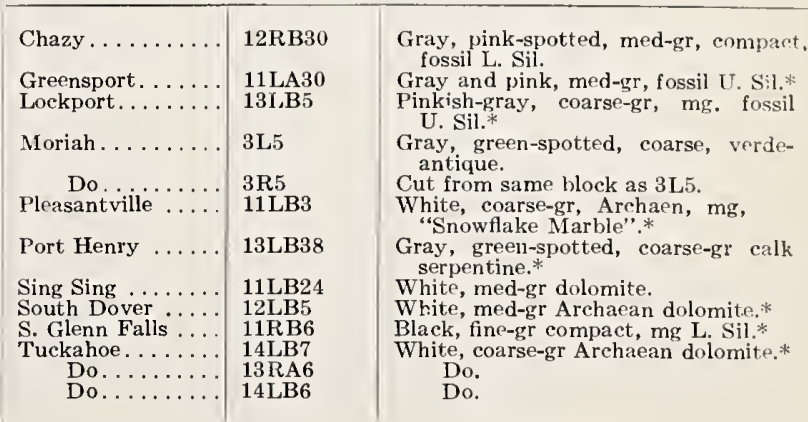


TABLE 1. Source, classification, and description of domestic stones in south face of wall-Continued

\begin{tabular}{c|c|c|c|c|}
$\begin{array}{c}\text { Specific } \\
\text { source } \\
\text { No. }\end{array}$ & Nescription \\
New York Quartzite & Doll
\end{tabular}

\begin{tabular}{l|l|l|l}
\hline Fort Ann ....... & 9LB32 & Buff, fine-gr, compact.*
\end{tabular}

New York Sandstone

See also S-2, $9 \mathrm{~S}-2,131$, and $21 \mathrm{EE}$ of tahle 4

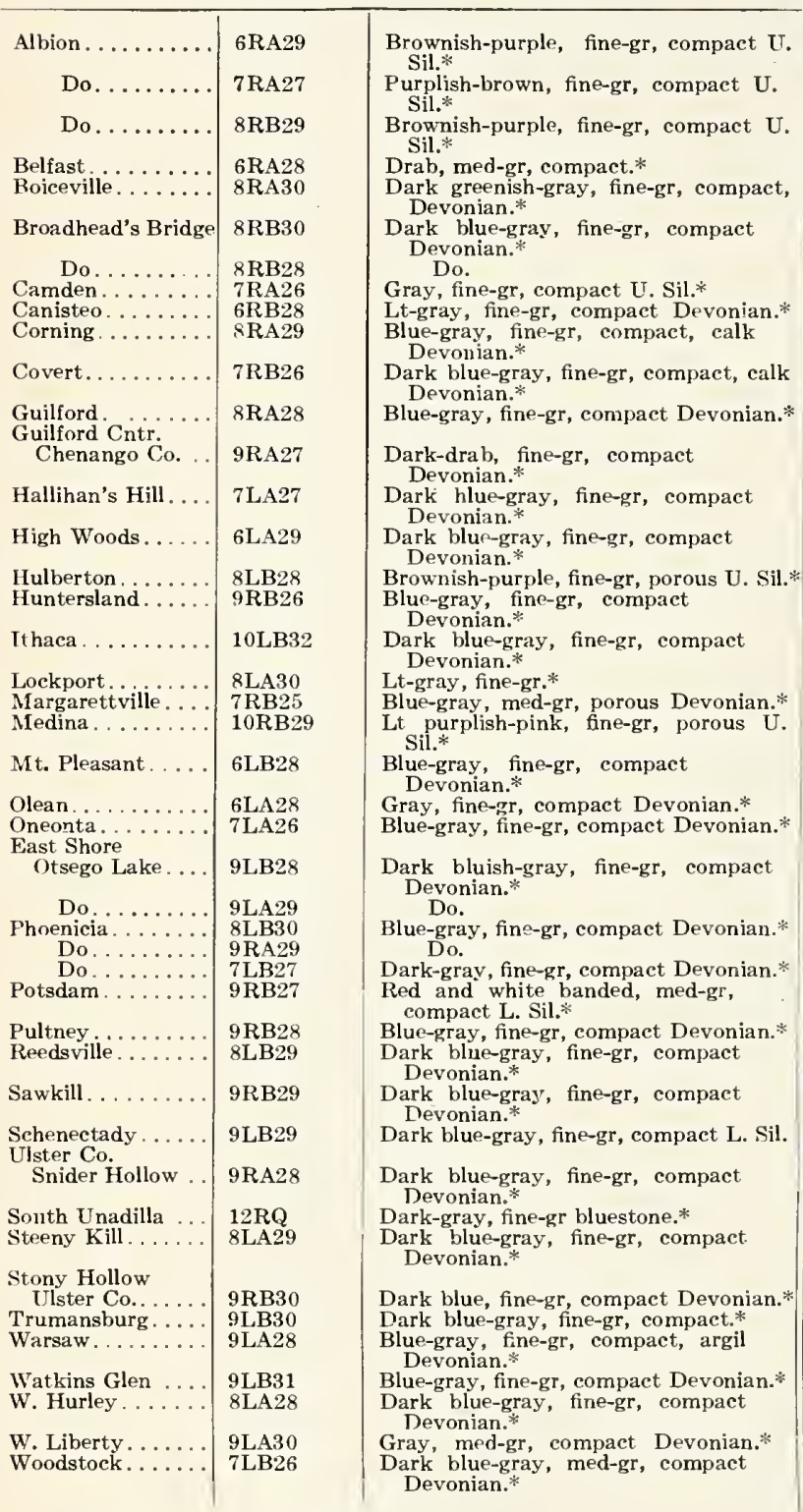

North Carolina Dolomite

Nottla......... 12 LB2 $\quad$ Lt-blue, fine-gr, compact Archaean.

\section{North Carolina Gneiss}

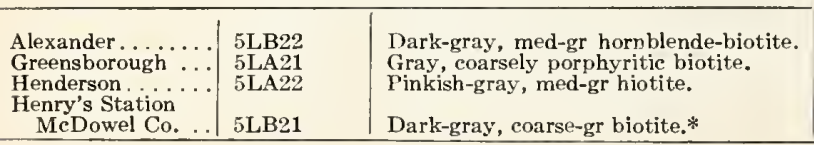

\begin{tabular}{|c|c|c|}
\hline $\begin{array}{l}\text { Specific } \\
\text { source }\end{array}$ & $\begin{array}{l}\text { Wall } \\
\text { No. }\end{array}$ & Description \\
\hline $\begin{array}{l}\text { Hickory ......... } \\
\text { Jamestown ...... } \\
\text { Mooresville ..... } \\
\text { Morganton Depot. } \\
\text { Raleigh.......... } \\
\text { Shelby ......... }\end{array}$ & $\begin{array}{l}\text { 11LB42 } \\
5 \mathrm{LB} 20 \\
6 \mathrm{LB} 23 \\
6 \mathrm{LA} 23 \\
8 \mathrm{LB} 25 \\
7 \mathrm{LB} 22\end{array}$ & $\begin{array}{l}\text { Gray, coarse-gr biotite.* } \\
\text { Do. } \\
\text { Red, yellow-mottled, fine-gr.* } \\
\text { White, green-spotted, fine-gr, horn- } \\
\text { blende.* } \\
\text { Lt blue-gray, fine-gr biotite. } \\
\text { Gray, fine-gr biotite.* }\end{array}$ \\
\hline \multicolumn{3}{|c|}{$\begin{array}{l}\text { North Carolina Granite } \\
\text { See also S-2 of table } 4\end{array}$} \\
\hline
\end{tabular}

\begin{tabular}{l|l|l}
\hline Alamance Co. .... & 8LB24 & Gray, fine-gr hiotite granite.
\end{tabular}

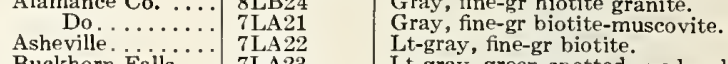

Buckhorn Falls ... 7LA23 Lt-gray, green-spotted, med-gr biotite.*

Charlotte...... 8LB26 Gray, med-gr biotite.

\begin{tabular}{r|l|l} 
Do .......... & 6LB24 & Gray, coarse-gr biotite. \\
Concord........ & 6LA24 & Lt-red, fine-gr biotite.
\end{tabular}

\begin{tabular}{c|c} 
Contentnea Cr. & \\
Wilson Co. .... & 7LB21
\end{tabular}

\begin{tabular}{r|r} 
Wilson Co...... & 7LB21 \\
Danbury ....... & $8 \mathrm{LB} 23$
\end{tabular}

Davidson College 5 LA23

Do College . $5 \mathrm{LA} 23$

Gaston Co.........

Gastonia.......... 7 RA23

Greensborough .... 8 RB23

Hillsboro........ 8 8RB26

$\begin{array}{ll}\text { Lexington. ........... } & 8 \text { RB25 } \\ \text { Louisburg } & \text { 10LB27 }\end{array}$

Louisburg .........

Mecklenburg Co... 5RA25

Mooresville..... 5 RB2

itt. Airy . . . . . . . $6 \mathrm{RB} 23$

Do.

Mt. Monroe......... 5 5RB22

Oxford .......... 8RB24

Poison Springs.....

Rockingham..... 5RA23

Do.......... 5RA24

Salisbury ....... 6RB24

Do.......... 6RA23

Do......... 6RA24

Toisnot......... ${ }_{5 \mathrm{RB} 21}^{6 \mathrm{~B}}$

Warren Plains..... 7RB22

Warrenton...... 5RB24

Pink, coarse-gr, porphyritic biotite.

Pinkish-gray, coarse-gr hiotite.

Dark-gray, med-gr hornblende-biotite.*

Gray, coarse-gr biotite.*

Gray, coarse-gr biotite.*

Gray, fine-gr muscovite

Pray, fine-gr biotite.

Pink, fine-gr biotite.*

Gray, fine-gr biotite.
Gray, pink-spotted, fine-gr biotite

Lt-gray, fine-gr biotite.*

Gray, coarse-gr hornblende.*

Gray, fine-gr biotite.*

Yellowish-gray, med-gr biotite.*

Lt-gray, med-gr biotite, "Mt. Airy".*

Gray, coarsely prophyritic biotite "

Dark-gray, coarse-gr biotite.*

Grav, med-gr biotite*

Greenish-gray, coarse-gr biotite.

Pinkish-gray, coarse-gr prophyritic

Lt-gray, fine-gr Archaean biotite.*

Pinkish-gray, eoarse-gr biotite.*

Brownish-gray, med-gr biotite.*

Pink, med-gr biotite "Salisbury".**

Gray, pink-spotted, med-gr biotite.*

White, fine-gr muscovite.

Lt-gray, fine-gr biotite.

Gray, coarse-gr biotite.*

North Carolina Limestone

\begin{tabular}{l|l|l} 
Newbern........ & 9RB5 & $\begin{array}{c}\text { Buff, coarse-gr, coarsely cellular } \\
\text { Eocene. }\end{array}$
\end{tabular}

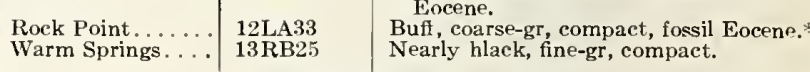

North Carolina Marble

See also S-2, 162 of table 4

\begin{tabular}{|c|c|c|}
\hline $\begin{array}{l}\text { Murphy .......... } \\
\text { Nantahala ...... } \\
\text { Red Marble Gap... } \\
\text { Valley Town ..... }\end{array}$ & $\begin{array}{l}\text { 9LB4 } \\
12 \mathrm{LB} 34 \\
10 \mathrm{RB} 19 \\
9 \mathrm{RB} 4\end{array}$ & $\begin{array}{l}\text { Dark-blue, fine-gr Archaean calc.* } \\
\text { Lt-pink, fine-gr Archaean calc. } \\
\text { Pink, fine-gr Archaean calc.* } \\
\text { Blue-hlack, fine-gr Archaean calc.* }\end{array}$ \\
\hline
\end{tabular}

Red Marble Gap 10RB1

Blue-hlack, fine-gr Archaean calc**

\begin{tabular}{|l|l|l}
\hline \multicolumn{3}{|c}{ North Carolina Quartz Porphyry } \\
\hline Charlotte ....... & 7LB23 & White, dark-spotted, "Leopard Rock".* \\
\hline
\end{tabular}

North Carolina Quartzite

\begin{tabular}{l|l|l} 
Hot Springs...... & 7RA22 & Purple, fine-gr, compact.
\end{tabular}

\section{North Carolina Sandstone}

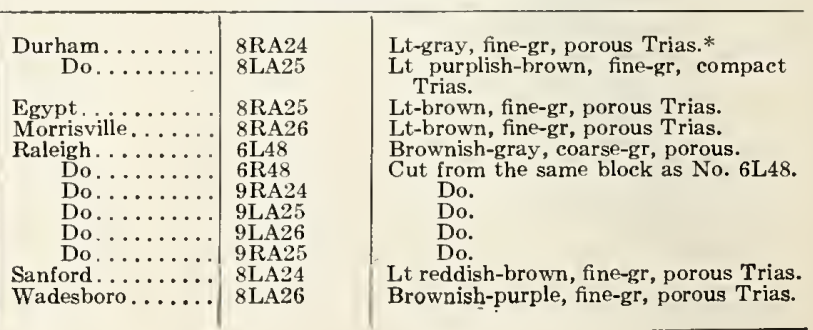


TABLE 1. Source, classification, and description of domestic stones in south face of wall-Continued

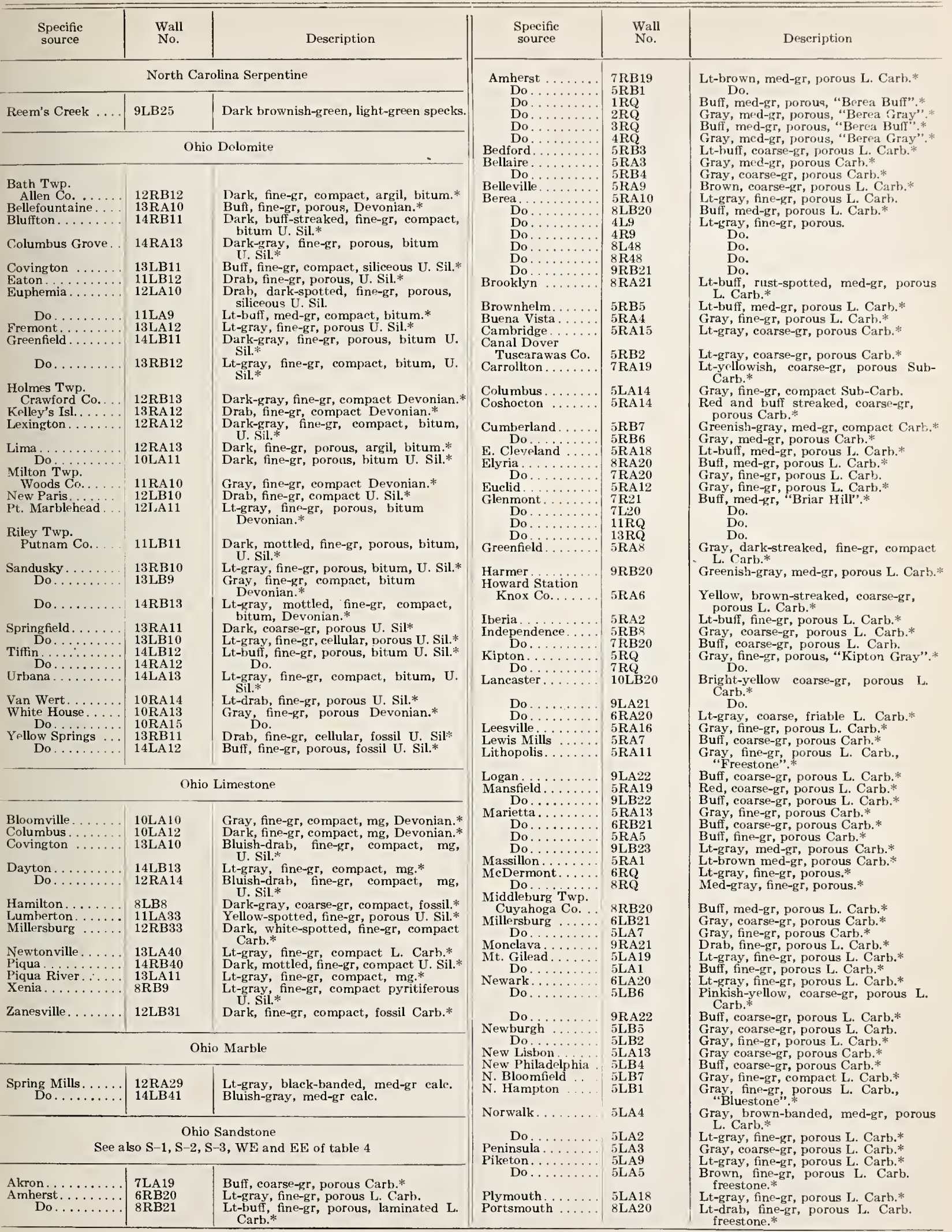


TABLE 1. Source, classification, and description of domestic stones in south face of wall-Continued

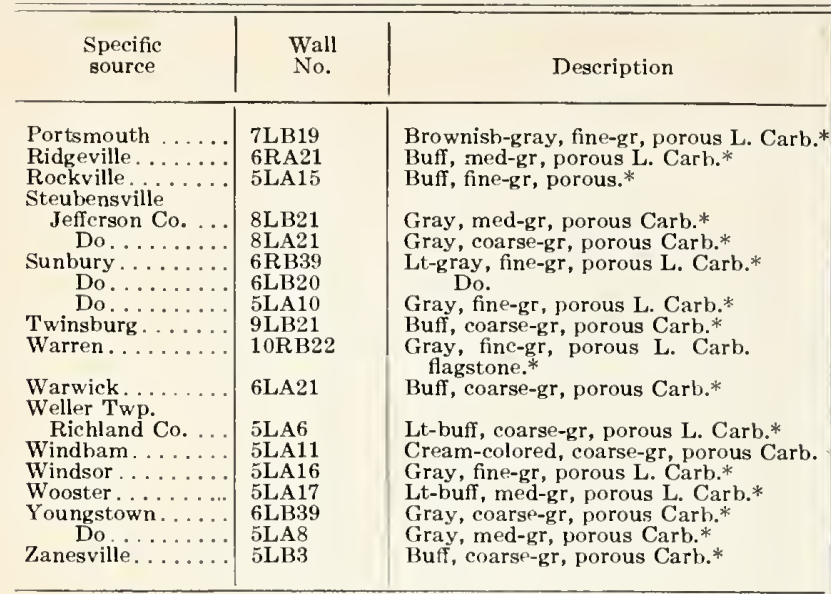

Oklahoma Limestone

\begin{tabular}{l|l|l}
\hline Cherokee ....... & 9LB $5 \overline{ }$ & Pale-buff, med-gr, semicryst, compact.
\end{tabular}

Oregon Basalt

\begin{tabular}{c|l|l}
\hline $\begin{array}{c}\text { Alhany............ } \\
\text { Clackamas Station } \\
\text { Clackamas Co. }\end{array}$ & 10RB42 & Dark-gray, med-gr, compact. \\
& Gray, fint-gr, porous.*
\end{tabular}

\begin{tabular}{l|l|l}
\hline \multicolumn{3}{c}{ Oregon Diabase } \\
\hline Albany region .... & 9LB39 & Gray, med-gr, compact.* \\
Asbland....... & 9LB38 & Nearly black, fine-gr, compact. \\
\hline
\end{tabular}

\begin{tabular}{|c|c|c|}
\hline \multicolumn{3}{|c|}{ Oregon Sandstone } \\
\hline 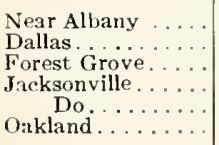 & $\begin{array}{l}\text { 9RB33 } \\
11 \mathrm{RB} 34 \\
9 \mathrm{LB} 37 \\
11 \mathrm{RB} 33 \\
9 \mathrm{LB} 34 \\
9 \mathrm{RB} 37\end{array}$ & $\begin{array}{l}\text { Brown, fine-gr, porous.* } \\
\text { Gray-brown fine-gr, porous. } \\
\text { Gray, fine-gr, porous. } \\
\text { Gray, fine-gr, porous. } \\
\text { Reddish-brown, fine-gr, porous. } \\
\text { Blue-gray, fine-gr, compact, argil.* }\end{array}$ \\
\hline \multicolumn{3}{|c|}{ Pennsylvania Argillite (Slate) } \\
\hline 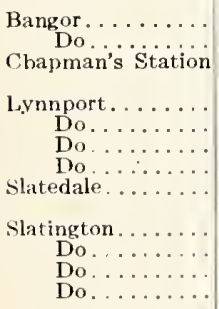 & $\begin{array}{l}\text { 8LA18 } \\
\text { 6RB15 } \\
\text { 9LB19 } \\
\text { 10LB18 } \\
\text { 10L51 } \\
\text { 10R51 } \\
\text { 9RA17 } \\
\text { 7RA17 } \\
\text { 10RB21 } \\
\text { 7LA17 } \\
\text { 6LB15 } \\
\text { 10RB20 }\end{array}$ & $\begin{array}{l}\text { Blue-black, fine-gr, roofing slate.* } \\
\text { Do. } \\
\text { Blue-black, fine-gr, banded roofing } \\
\text { slate.* } \\
\text { Dark-gray, fine-gr slate. } \\
\text { Cut from same hlock as 10LB18. } \\
\text { Do. } \\
\text { Dark-gray, fine-gr, slate. } \\
\text { Blue-black, fine-gr roofing and tile } \\
\text { slate.* } \\
\text { Do. } \\
\text { Do. } \\
\text { Do. } \\
\text { Do. }\end{array}$ \\
\hline
\end{tabular}

\begin{tabular}{l|l|l}
\hline \multicolumn{3}{c}{ Pennsylvania Conglomerate } \\
\hline $\begin{array}{l}\text { Fairfield......... } \\
\text { Friederisburg .... }\end{array}$ & 9LB20 & $\begin{array}{l}\text { Red and gray, calk conglomerate. } \\
\text { Dark purple, coarsc-gr, porous, } \\
\text { siliceous Potsdam.* } \\
\text { Peddish-gray, compact comglomerate.* } \\
\text { Prown and gray, coarse, compact, } \\
\text { siliceous. }\end{array}$ \\
\hline
\end{tabular}

Pennsylvania Diabase

\begin{tabular}{|c|c|c|}
\hline Collins Station. . & & \\
\hline Lancaster Co. & 6LB14 & Dark-gray, fine-gr, Mesozoic.* \\
\hline $\begin{array}{l}\text { Gettysburg..... } \\
\text { Goldsboro. }\end{array}$ & $\begin{array}{l}\text { 6LB16 } \\
8 \mathrm{LA} 19\end{array}$ & $\begin{array}{l}\text { Gray, fine-gr.* } \\
\text { Do. }\end{array}$ \\
\hline Jacksonwald: & $6 \mathrm{LB} 19$ & Dark-gray, fine-gr. \\
\hline$\underset{\text { Do }}{\text { Reading ..... }}$ & $\begin{array}{l}\text { 6RB14 } \\
6 \mathrm{RB} 19\end{array}$ & $\begin{array}{l}\text { Nearly black, fine-gr. } \\
\text { Dark-gray, coarse-gr. }\end{array}$ \\
\hline Round Top & & \\
\hline $\begin{array}{r}\text { Gettysburg } \\
\text { St. Peters... }\end{array}$ & $\begin{array}{l}\text { 6RB16 } \\
\text { 6RB10 }\end{array}$ & $\begin{array}{l}\text { Gray, fine-gr, porous Mesozoic. } \\
\text { Dark-gray, med-gr, compact, } \\
\text { "St. Peters Granite".* }\end{array}$ \\
\hline
\end{tabular}

\begin{tabular}{|c|c|c|}
\hline $\begin{array}{l}\text { Specific } \\
\text { source }\end{array}$ & $\begin{array}{l}\text { Wall } \\
\text { No. }\end{array}$ & Description \\
\hline
\end{tabular}

\begin{tabular}{l|l|l}
\hline Reading....... & $8 \mathrm{LB} 19 \quad \begin{array}{c}\text { Black and white-spotted, coarse-gr, } \\
\text { slightly porous. }\end{array}$
\end{tabular}

Pennsylvania Dolomite

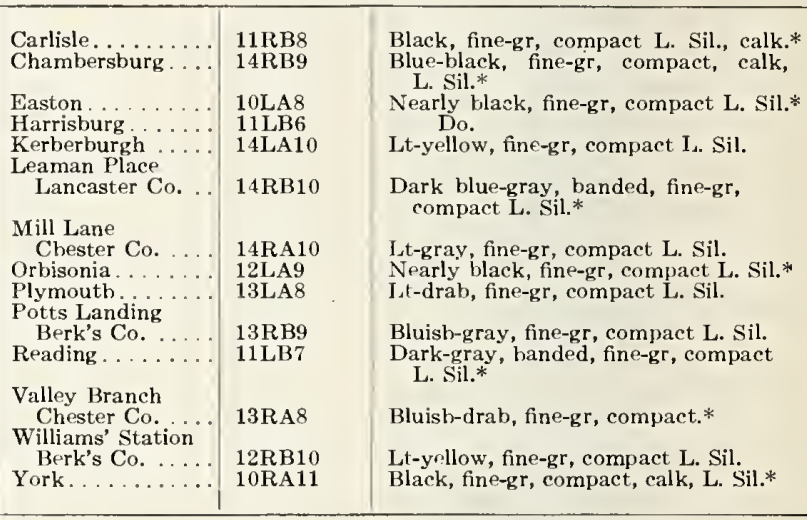

Pennsylvania Gneiss

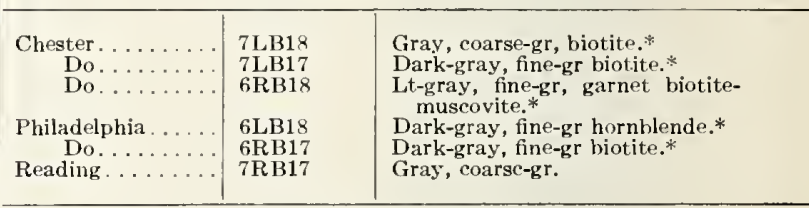

Pennsylvania Granite

\begin{tabular}{|l|l|l}
\hline Allentown...... & $6 \mathrm{LB17}$ & $\begin{array}{l}\text { Gray, red-spotted, med-gr hornblende- } \\
\text { biotite. } \\
\text { Gray-mottled, fine-gr hornblende- } \\
\text { biotitc. }\end{array}$ \\
\hline
\end{tabular}

Pennsylvania Limestone

\begin{tabular}{|c|c|c|}
\hline $\begin{array}{l}\text { Connellsville. } \\
\text { Cove Station }\end{array}$ & 7RA14 & Lt-blue, med-gr, compact L. Carb.* \\
\hline Bedf & $11 \mathrm{RA} 8$ & $\begin{array}{l}\text { Gray, coarse-gr, compact, mg, cry } \\
\text { Devonian.* }\end{array}$ \\
\hline East Conshobocken & 7LA13 & $\begin{array}{l}\text { Blue-gray, med-gr, compact, } \\
\text { micaceous L. Sil. }\end{array}$ \\
\hline
\end{tabular}

Flourtown $\ldots . \ldots$ 14LB9 $^{\text {Lt-yellow, blue-spotted, fine-gr, }}$

Harrisburg. ..... 7 7RA13 $\begin{gathered}\text { compact L. Sil. } \\ \text { Dark blue-gray, white-veined, fine-gr, }\end{gathered}$

compact L. Sil.

Hummelstown.... 7LA12 Blue, fine-gr, compact L. Sil.

Huntingdon..... 12RB32 Nearly black, fine-gr, compact.*

Hyndman .... 14LB39 Black, fine-gr, compact.*

Lebanon......... 7RA15 Nearly hlack, fine-banded, fine-gr,

\begin{tabular}{r|r} 
Do........ & 7LA16 \\
Do....... & 7LA15
\end{tabular}

\begin{tabular}{r|r} 
Do......... & 7LA15 \\
MRB17
\end{tabular}

\begin{tabular}{ll|l} 
Mertztown ........ & $8 \mathrm{RB} 17$ \\
Morrell ........ & 13RB 8
\end{tabular}

Myerstown. .... 13RB37

\begin{tabular}{ll|l} 
N. Annville & $\ldots \ldots$ & $13 \mathrm{RB} 36$ \\
Palmyra. & & $14 \mathrm{LB3} 8$
\end{tabular}

Paxton Station

\begin{tabular}{c|c} 
Paxton Station & $13 \mathrm{LB} 39$
\end{tabular}

Daupbin Co.

\begin{tabular}{l|l} 
Lehand Station & \\
Lehanon ... & $8 \mathrm{LB17}$
\end{tabular}

\begin{tabular}{r|l} 
Do............ & 13RA37
\end{tabular}

\begin{tabular}{c|c} 
Do............... & 13RA37 \\
$\begin{array}{c}\text { Spruce Creek } \\
\text { Huntingdon Co. }\end{array}$ & 14LA9 \\
Vanport......... & 14RA9
\end{tabular}

\begin{tabular}{l|l} 
Wernersville...... & 7LA14
\end{tabular}

Do........ 7RA16
Nearly hlack, fin

Dark blue-gray, fine-gr, compact L. Sil.
Dark blue-gray, fin $\mathrm{P}-\mathrm{gr}$, compact L. Sil. Dark blue-gray, fin ${ }^{\rho-g r}$, compact L. Sil.
Do. Do.
Dark-gray, coarse-gr, compact, mg L. Sil.*
Blue-gray, fine-gr, compact L. Sil.

Blue-gray, fine-gr, compact L. Sil.
Dark blue-gray, fine-gr, compact L. Sil. Do.

Do.

Blue-gray, fine-gr, compact, cryst $\mathbf{L}$. Dil.

Black, fine-gr, compact, mg L. Sil* Dark-gray, coarse-gr, compact, fossil, mg Carb.*

Water-blue, dark-veined, fine-gr, compact, cryst I. Sil.

Dark blue-gray, fine-gr, compact, 
TABLe 1. Source, classification, and description of domestic stones in south face of wall-Continued

\begin{tabular}{l|l|l|l}
\hline $\begin{array}{c}\text { Specific } \\
\text { source }\end{array}$ & $\begin{array}{l}\text { Wall } \\
\text { No. }\end{array}$ & Description \\
\hline
\end{tabular}

Pennsylvania Marble

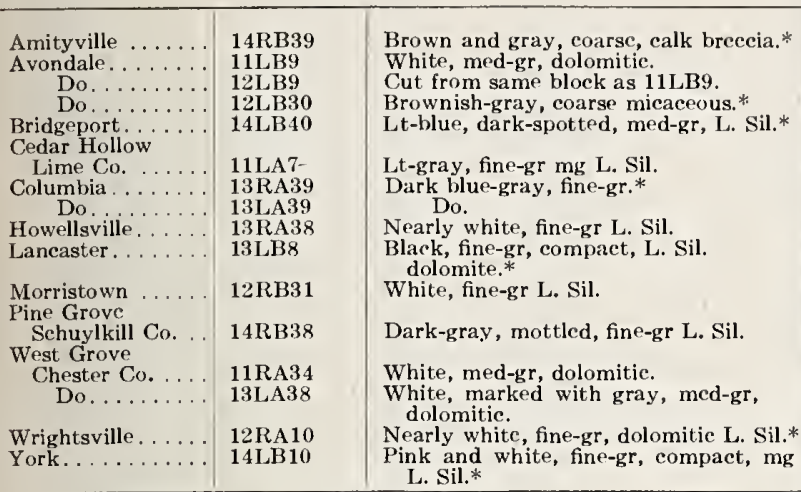

Pennsylvania Sandstone

Sce also S-1, EE, and WE of table 4

\begin{tabular}{|c|c|c|}
\hline Altoona & $6 \mathrm{LA} 8$ & $\begin{array}{l}\text { Buff, gray-streaked, fine-gr, compact } \\
\text { L. Carb. } *\end{array}$ \\
\hline $\begin{array}{l}\text { Antrim } \\
\text { Baden. }\end{array}$ & $\begin{array}{l}\text { 9LA18 } \\
\text { 6LB9 }\end{array}$ & $\begin{array}{l}\text { Lt-gray, coarse, cellular Carh.* } \\
\text { Gray, rust-spotted, coarse, porous } \\
\text { Carb. } *\end{array}$ \\
\hline $\begin{array}{l}\text { Beaver Falls. } \\
\text { Black Walnut. }\end{array}$ & $\begin{array}{l}6 \mathrm{LB13} \\
6 \mathrm{RB} 8\end{array}$ & $\begin{array}{l}\text { Lt-gray, coarse-gr, porous Carb.* } \\
\text { Lt blue-gray, fine-gr, porous Devonian.* }\end{array}$ \\
\hline Cann & $6 \mathrm{RB} 7$ & Lt-gray, fine-gr, porous Carb.* \\
\hline $\begin{array}{l}\text { Center Bridge } \\
\text { Do...... }\end{array}$ & $\begin{array}{l}6 \mathrm{RB} 6 \\
6 \mathrm{RB} 4\end{array}$ & $\begin{array}{l}\text { Purplish-gray, fine-gr, compact Trias.* } \\
\text { Gray, rust-spotted, coarse-gr, compact } \\
\text { Trias.* }\end{array}$ \\
\hline Columbia . & 6RA2 & $\begin{array}{l}\text { Blue-drab, fine-gr, compact, argil L. } \\
\text { Sil.* }\end{array}$ \\
\hline Connerllsville & 6RA15 & $\begin{array}{l}\text { Green-tinted, brown, coarsc, porous } \\
\text { Carb.* }\end{array}$ \\
\hline Do..... & $\begin{array}{l}\text { 6RA4 } \\
8 \mathrm{RB} 19\end{array}$ & $\begin{array}{l}\text { Lt-gray, fine-gr, porous Carb.* } \\
\text { Lt-gray, fine-gr, compact Sub-Carb.* }\end{array}$ \\
\hline & 9LA19 & Buff-tinted, coarse-gr, porous.* \\
\hline & $6 \mathrm{RA}$ & $\begin{array}{l}\text { Dark blue-gray, fine-gr, compart U. } \\
\text { Sil. }\end{array}$ \\
\hline $\begin{array}{l}\text { Derry Station } \\
\text { Westmorland } \sigma\end{array}$ & $6 \mathrm{RA} 7$ & Bluish-gray, fine-gr, compact Carb.* \\
\hline Erie.... & & Drab, fine-gr, compact Devonian.** \\
\hline $\begin{array}{l}\text { Farrandsville. } \\
\text { Fayette Statio }\end{array}$ & $6 \mathrm{R}$ & Buff, fine-gr, porc \\
\hline Fayette Co. & $8 R B 18$ & Lt pinkish-buff, coarse-gr, porous.* \\
\hline$D$ & 9RA19 & $\begin{array}{l}\text { Gray, fine-gr, porous Sub-Carb.* } \\
\text { Greenish-gray, fine-gr, porous, Suh- } \\
\text { Carb.* }\end{array}$ \\
\hline Fuller & $6 R A 14$ & Buf, coarse-gr, porous Carb.* \\
\hline & $\begin{array}{l}\text { 6RA9 } \\
\text { 6RA16 }\end{array}$ & $\begin{array}{l}\text { Yellow, coarse-gr, poro } \\
\text { Purplish-brown, med-gr }\end{array}$ \\
\hline & & Purplish-brown, med-gr, conpact Trias. \\
\hline $\begin{array}{l}\text { Greer } \\
\text { Greer }\end{array}$ & $\begin{array}{l}6 \mathrm{R} A \\
6 \mathrm{RI}\end{array}$ & $\begin{array}{l}\text { Gray, f } \\
\text { Lt-olive }\end{array}$ \\
\hline Homer & $9 \mathrm{LB}$ & -gr, porous Carb." \\
\hline & & $\begin{array}{l}\text { Brown, coarse-gr, porous Carb.* } \\
\text { Brownish-purple, coarse-gr, porous }\end{array}$ \\
\hline Hummelstow & 6RA3 & $\begin{array}{l}\text { Brownish-purple, coarse-gr, porous } \\
\text { Trias.* }\end{array}$ \\
\hline Johnstown. & 6RA10 & Lt-gray, fine-gr, compact Carb.* \\
\hline & 6RB3 & Gray, coarse-gr, porous Carb.* \\
\hline Lay & & Lt-gra \\
\hline & & Lt-brown, med-gr, compact Devonian.* \\
\hline & & $\begin{array}{l}\text { Gray, dark-streaked, med-gr, compact } \\
\text { Carb.* }\end{array}$ \\
\hline Lumberton. . & 8RA19 & Lt brownish-purple, fine-gr, compact \\
\hline $\begin{array}{l}\text { Mainesburgh ... } \\
\text { Mauch Chunk. . }\end{array}$ & $\begin{array}{l}\text { 6RA1 } \\
\text { 6RA5 }\end{array}$ & $\begin{array}{l}\text { Blue-gray, fine-gr, compact Devonian.* } \\
\text { Dark-gray, fine-gr, compact. }\end{array}$ \\
\hline & & Gray, coarse-gr, compact Carh. \\
\hline & $\begin{array}{l}\text { 9RA18 } \\
\text { 6LA13 }\end{array}$ & $\begin{array}{l}\text { Buff, coarse-gr, porous, friable Carb.* } \\
\text { Buff, coarsc-gr, porous Carb.* }\end{array}$ \\
\hline Mes & $6 \mathrm{LA}$ & Blue-gray, fine-gr, compact Devonian.* \\
\hline & & $\begin{array}{l}\text { Lt blue-gray, fine-gr, compact } \\
\text { Devonian.* }\end{array}$ \\
\hline $\begin{array}{l}\text { Norristown. } \\
\text { Oil City. }\end{array}$ & $6 \mathrm{LB12}$ & $\begin{array}{l}\text { Reddish-brown, coarse, porous Trias.* } \\
\text { Buf, fine-gr, porous Carb.* }\end{array}$ \\
\hline & & $\begin{array}{l}\text { But, } \\
\text { Pink, }\end{array}$ \\
\hline & & Lt-olive, finc-gr, porous Carb.* \\
\hline & & Dark-brown, banded, fine-gr, compact \\
\hline Pond Eddy & 9LA20 & Dark bluc-gray, fine-gf, compact \\
\hline Prospect. & 6LB6 & Gray, fine-gr, compact Carb.* \\
\hline
\end{tabular}

\begin{tabular}{|c|c|c|}
\hline $\begin{array}{l}\text { Specific } \\
\text { source }\end{array}$ & $\begin{array}{l}\text { Wall } \\
\text { No. }\end{array}$ & Description \\
\hline Queens Run & & \\
\hline $\begin{array}{r}\text { Clinton Co. } \\
\text { Reading.......... }\end{array}$ & $\begin{array}{l}\text { 6LA18 } \\
6 \mathrm{LA12}\end{array}$ & $\begin{array}{l}\text { Dark-gray, finc-gr, compact Devonian.* } \\
\text { Brownish-purple, med-gr, porous }\end{array}$ \\
\hline $\begin{array}{l}\text { Seranton } \ldots \ldots \\
\text { Do . . . . }\end{array}$ & $\begin{array}{l}\text { 6LA5 } \\
\text { 6LA11 }\end{array}$ & $\begin{array}{l}\text { Gray. med-gr, porous, Sub-Carh.* } \\
\text { Buff-banded, coarse-gr, compact }\end{array}$ \\
\hline $\begin{array}{l}\text { Seottdale } \ldots \ldots \\
\text { Do....... }\end{array}$ & $\begin{array}{l}\text { 6LA6 } \\
9 \mathrm{RB} 18\end{array}$ & $\begin{array}{l}\text { Devonian.* } \\
\text { Brown-handed, fine-gr, compact Carh.* } \\
\text { Do. }\end{array}$ \\
\hline Sharon .... & $6 \mathrm{LB11}$ & Buff, coarse-gr, porous Carh.* \\
\hline $\begin{array}{l}\text { Leneksnnny } \\
\text { Luzerne Co. . } \\
\text { Do, . B } \\
\text { Skinner's Eddy }\end{array}$ & $\begin{array}{l}\text { 9LA17 } \\
6 \mathrm{LB} 4 \\
6 \mathrm{LA17}\end{array}$ & $\begin{array}{l}\text { Rluc-gray, finc-gr, compact Carb.* } \\
\text { Dark-gray, coarse-gr, compact Carh.* } \\
\text { Lt blue-gray, fine-gr, compact }\end{array}$ \\
\hline Stoncboro... & 6LA10 & $\begin{array}{l}\text { Devonian,* } \\
\text { Lt-brown, coarse-gr, porous Carb, }\end{array}$ \\
\hline Stoop's Ferry & 10LB 19 & Gray, fine-gr, porous, argil Carb.* \\
\hline $\begin{array}{l}\text { Iitusville... } \\
\text { Uniontown. }\end{array}$ & $\begin{array}{l}\text { 6LA16 } \\
9 \mathrm{RA} 20\end{array}$ & $\begin{array}{l}\text { Lt-but, the-gr, porous Carb. } \\
\text { Buff, fine-gr, porous Sub-Carb.* }\end{array}$ \\
\hline Wampum... & 6LA4 & Buff, coarse-gr, porous Carb." \\
\hline Warren...... & $\begin{array}{l}\text { 6LB10 } \\
6 \mathrm{LA} 3\end{array}$ & $\begin{array}{l}\text { Brown, fine-gr, compact Carl,.* } \\
\text { Lt-gray, med-gr, porous Carb. }\end{array}$ \\
\hline Washington. & $6 \mathrm{LA} 15$ & Yellowish-brown, coarse-gr, porous \\
\hline $\begin{array}{l}\text { Waynesburgh. } \\
\text { Webstcr. } \\
\text { Yardleyvile... }\end{array}$ & $\begin{array}{l}\text { 6LA9 } \\
\text { 6LB8 } \\
\text { 6LB7 }\end{array}$ & $\begin{array}{l}\text { Buff, coarse-gr, porous Carl).* } \\
\text { Brownish-gray, banded, fine-gr, Carb.* } \\
\text { Lt-hrown, med-gr, porous Trias.* }\end{array}$ \\
\hline
\end{tabular}

Pennsylvania Quartz

\begin{tabular}{l|l|l}
\hline Pine Grove ...... & 6LA19 & Dull-red, coarse-gr, quartz porphyry.
\end{tabular}

Pennsylvania Quartzitc

\begin{tabular}{l|l|l}
\hline $\begin{array}{l}\text { Huntingdon. ..... } \\
\text { Jacksonwald .... }\end{array}$ & 6RB1 & 6RA19 \\
Mapleton........ & TLA18 & Lt-gray, fine-gr.* \\
& Lt-gray, fine-gr, compact.* \\
\hline
\end{tabular}

Pennsylvania Serpentine

\begin{tabular}{|c|c|c|}
\hline $\begin{array}{l}\text { Chester.... } \\
\text { W. Chester } \\
\text { Do . . }\end{array}$ & $\begin{array}{l}\text { 6RB9 } \\
10 \mathrm{LB16} \\
8 \mathrm{LB18}\end{array}$ & $\begin{array}{l}\text { Green, coarse-gr, impure.* } \\
\text { Do. } \\
\text { Do. }\end{array}$ \\
\hline
\end{tabular}

Pennsylvania Soapstone

\begin{tabular}{r|l|l}
\hline Montgomery Co... & $10 \mathrm{LB17}$ & $\begin{array}{c}\text { Gray, dark-mottled, coarse-gr, } \\
\text { compact.* } \\
\text { Do. }\end{array}$ \\
Do . . . . . . & $7 \mathrm{RB16}$ &
\end{tabular}

Rhode Island Gneiss

Diamond Hill.... 10 LA4 8 Greenish-gray, fine-gr, hornblende.*

Rhode Island Granite

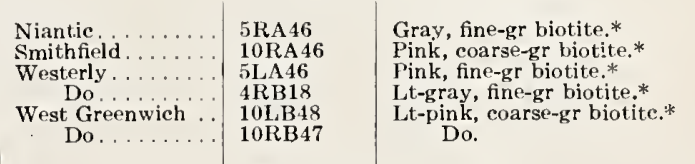

South Carolina Granite

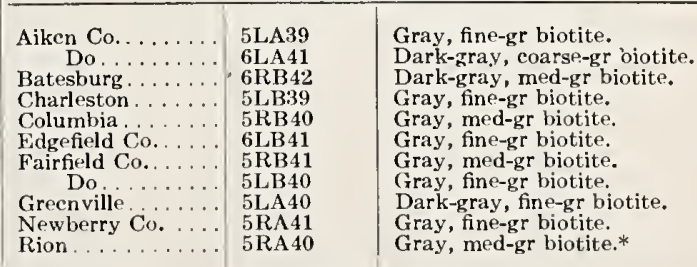


TABLE 1. Source, classification, and description of domestic stones in south face of wall-Continued

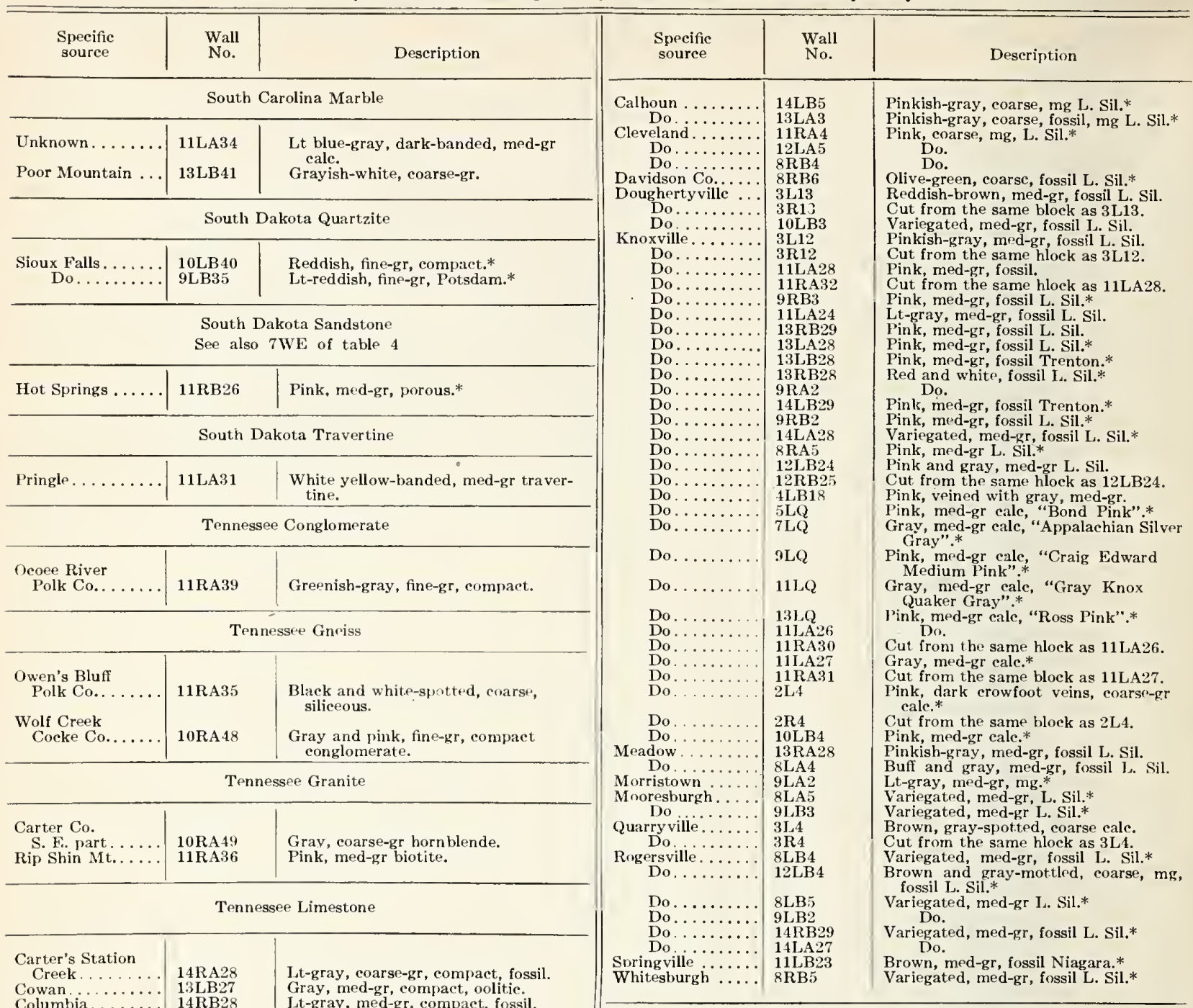

Cowan ......... 13LB27

Columbia. ..... 14RB28

Davidson Co... 14LB2R
Knoxville...... 12RA5

Do......... $4 \mathrm{LB} 11$

Do........ 4 RB11

Lehanon Pike .... 12RB24

Murfreesborough.. 12RB5

Nashville...... 11RA28

Do........ 13LA27

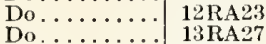

Do.......... 14RA27

\begin{tabular}{l|l} 
Nolensville Pike . . 10LA6 & 14
\end{tabular}

\begin{tabular}{r|l} 
Do & 8RA4 \\
Rogersville........ & 11LA25
\end{tabular}

Sherwood Station . 11RA29

Gray, med-gr, porous, fossil L. Sil.*

Brown, gray-mottled, coarse-gr,

compact, mg L. Sil.*

Pinkish-gray, med-gr, cryst, compact L. Sil.

Dark-gray, med-gr, compact, eryst,

fossil L. Sil.*

Dark-gray, fine-gr, compact, mg,

fossil L. Sil.

Gray, med-gr, compact, fossil, L. Sil. "Capitol Quarry".*

$$
\text { Do. }
$$

Black, fine-gr, compact, argil, fossil.*

Buff, coarse, cellular, fossil.*

Dark-gray, med-gr, compact, fossil L. Sil.*

Brownish-gray, fine-gr, compact, mg, fossil L. Sil.

Buff, fine-gr, porous $\mathbf{I}$. Sil.

Brown, gray-mottled, coarse-gr,

compact. L. Sil.*

Lt-gray, fine-gr, compact, oolitic.*

Tennessee Marble

Sec also $\mathrm{S}-2$, and EE of table 4

\begin{tabular}{|c|c|}
\hline Calhoun......... & 13RB3 \\
\hline Do... & 13RA3 \\
\hline Do........ & $13 \mathrm{LB} 3$ \\
\hline Do........ & $10 \mathrm{RA} 8$ \\
\hline
\end{tabular}

Pink, med-gr, mg L. Sil.*

Do.

Pink, buff-mottled, coarse, brecciated,
Tennessee Sandstone

See also $\mathrm{S}-1$ and $\mathrm{S}-2$ of table 4

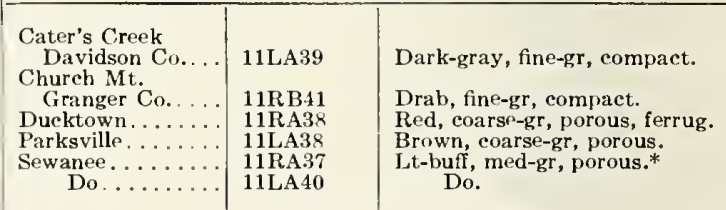

Tennessee Syenite

\begin{tabular}{c|c}
\hline $\begin{array}{c}\text { Hiwasee copper } \\
\text { mines......... }\end{array}$ & 10RA47 $\begin{array}{c}\text { Gray, green-spangled, med-gr horn- } \\
\text { blende. }\end{array}$ \\
\hline
\end{tabular}

Texas Diorite

\begin{tabular}{l|l|l} 
El Paso........ & 6RA32 & It greenish-gray, med-gr.
\end{tabular}

Texas Dolomite

Burnet............. 11RB2

San Saba.......... 14LA4
Dull-red, variegated, fine-gr, compact. Buff, fine-gr, compact. 
TABLE 1. Source, classification, and description of domestic stones in south face of wall-Continued

\begin{tabular}{|c|c|c|}
\hline $\begin{array}{l}\text { Specific } \\
\text { source }\end{array}$ & $\begin{array}{l}\text { Wall } \\
\text { No. }\end{array}$ & Description \\
\hline \multicolumn{3}{|c|}{$\begin{array}{c}\text { Texas Granite } \\
\text { See also } S-2,87 \text { of table } 4\end{array}$} \\
\hline $\begin{array}{l}\text { Burnet } \ldots \ldots \\
\text { Do....... } \\
\text { Kingsland... } \\
\text { Nr. Llano.... } \\
\text { Do..... } \\
\text { Do..... }\end{array}$ & $\begin{array}{l}\text { 6LB32 } \\
\text { 7RB29 } \\
9 \mathrm{RB} \\
5 \mathrm{LA} 30 \\
6 \mathrm{RB} 31 \\
6 \mathrm{LA} 32\end{array}$ & $\begin{array}{l}\text { Red, coarse-gr, biotite.* } \\
\text { Red, fine-gr biotite. } \\
\text { Pink, coarse-gr biotite.* } \\
\text { Gray, coarse-gr biotite. } \\
\text { Do. } \\
\text { Pink, med-gr biotite. }\end{array}$ \\
\hline
\end{tabular}

Texas Limestone

See also $\mathrm{S}-2,2,3,127$ and $2 \mathrm{WE}$ of table 4

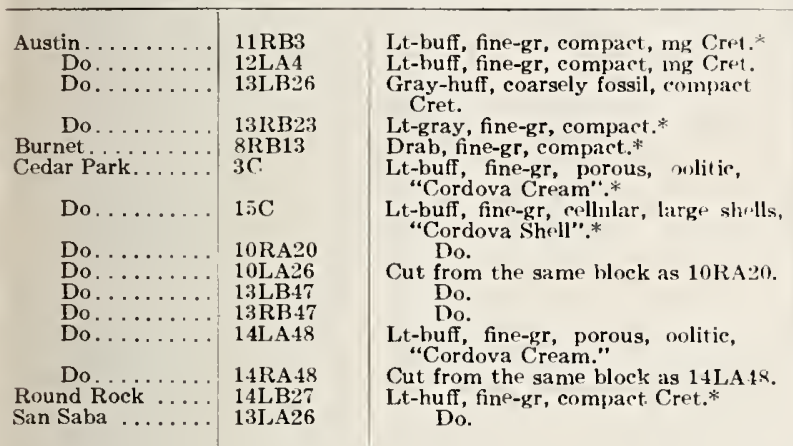

Texas Marble

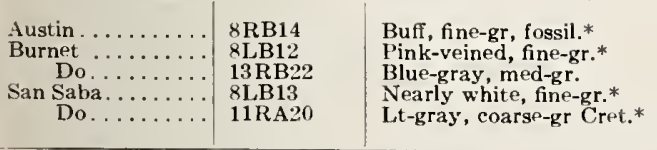

Texas Sandstone

\begin{tabular}{|c|c|c|}
\hline $\begin{array}{l}\text { Back Bone Valley } \\
\text { Burnet. ......... }\end{array}$ & $\begin{array}{l}\text { 8RB32 } \\
\text { 7RA30 }\end{array}$ & $\begin{array}{l}\text { Brown, coarse-gr, porous, ferrug L. Sil. } \\
\text { Brown, coarse-gr, compact. L. Sil., } \\
\text { calk. }\end{array}$ \\
\hline $\begin{array}{l}\text { Mormon Mills } \\
\text { Milsap........ }\end{array}$ & $\begin{array}{l}10 \mathrm{RB} 31 \\
7 \mathrm{RB} 30\end{array}$ & $\begin{array}{l}\text { Gray, med-gr, porous Carb., calk. } \\
\text { Reddish-brown, fine-gr, porous.* }\end{array}$ \\
\hline $\begin{array}{l}\text { Do. } \\
\text { P'ecos... }\end{array}$ & $\begin{array}{l}\text { 8RA32 } \\
\text { 8LA32 }\end{array}$ & $\begin{array}{l}\text { Do. } \\
\text { Red, fine-gr, compact. }\end{array}$ \\
\hline $\begin{array}{l}\text { Do. } \\
\text { Do. }\end{array}$ & $\begin{array}{l}10 \mathrm{LB} 33 \\
7 \mathrm{LB} 30\end{array}$ & Do. \\
\hline Do. & $7 \mathrm{LA30}$ & $\begin{array}{l}\text { Do. } \\
\text { Do. }\end{array}$ \\
\hline Range. & 9LA32 & Greenish-gray, fine-gr, poro \\
\hline & $8 \mathrm{LB} 32$ & Gray, coarse-gr, porous. \\
\hline
\end{tabular}

Utah Dolomite

\begin{tabular}{l|l|l} 
San Pete Valley ... & 11LB13 & $\begin{array}{c}\text { Dark, gray-mottled, fine-gr, compact, } \\
\text { fossil. }\end{array}$ \\
\hline Utah Limestone \\
\hline $\begin{array}{l}\text { Ephriam........ } \\
\text { Payson......... }\end{array}$ & 11RB B25 & $\begin{array}{l}\text { Lt-gray, fine-gr, porous, "San Pete } \\
\text { Stone".* } \\
\text { Drab, fine-gr, compact. }\end{array}$ \\
\hline
\end{tabular}

Utah Marble

\begin{tabular}{r|l|l}
$\begin{array}{r}\text { Beaver Co...... } \\
\text { Clinton....... }\end{array}$ & $\begin{array}{l}12 \mathrm{LB11} \\
8 \mathrm{LB14}\end{array}$ & $\begin{array}{l}\text { White, fine-gr calc. } \\
\text { Gray and brown variegated, coarsely } \\
\text { fossil calc.* } \\
\text { Buff, brown-mottled, fine-gr, fossil.* } \\
\text { Lt-gray, variegated, fine-gr calc. }\end{array}$ \\
\hline $\begin{array}{r}\text { Payson.......... } \\
\text { Utah Sandstone }\end{array}$ & $\begin{array}{l}13 \mathrm{LB} 36 \\
13 \mathrm{RB} 33\end{array}$ \\
\hline
\end{tabular}

\begin{tabular}{|c|c|c|}
\hline $\begin{array}{l}\text { Specifie } \\
\text { source }\end{array}$ & $\begin{array}{l}\text { Wall } \\
\text { No. }\end{array}$ & Description \\
\hline \multicolumn{3}{|c|}{ Vermont Argillite (Slate) } \\
\hline $\begin{array}{l}\text { Castleton. . . . . } \\
\text { Fair Haven ...... }\end{array}$ & $\begin{array}{l}5 \mathrm{RA44} \\
5 \mathrm{LA} 44\end{array}$ & $\begin{array}{l}\text { Blue-black, fine-gr, rough-texture } \\
\text { roofing slate.* } \\
\text { Red, finc-gr, rough-texture roofing and } \\
\text { tile. }\end{array}$ \\
\hline \multicolumn{3}{|c|}{$\begin{array}{l}\text { Vermont Granite } \\
\text { See also } S-2,47 \text { and } S-2,93 \text { of table } 4\end{array}$} \\
\hline 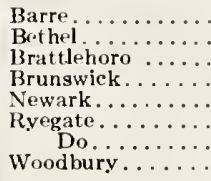 & $\begin{array}{l}\text { 6RB46 } \\
6 \mathrm{LB} 46 \\
6 \mathrm{LB45} \\
6 \mathrm{RB45} \\
6 \mathrm{RA45} \\
\text { 6RB47 } \\
\text { 5LA45 } \\
10 \mathrm{RA45}\end{array}$ & $\begin{array}{l}\text { Lt-gray, fine-gr biotite.* } \\
\text { While, med-gr muscovite.* } \\
\text { I,t-gray, fine-gr hiotite. } \\
\text { Gray, fine-gr biotite.* } \\
\text { Pink, coarse-gr biotite. } \\
\text { Gray, fine-gr biotite-muscovite.* } \\
\text { Gray, eoarse-gr hiotite-muscovite.* } \\
\text { Gray, med-gr biotite.* }\end{array}$ \\
\hline \multicolumn{3}{|c|}{ Vermont Limestone } \\
\hline
\end{tabular}

Isle La Motte .... 13 LA1 $\quad$ Dark-gray, fine-gr, fossil, mg.*

Vermont Marble

See also $\mathrm{S}-2,135$ to 138 of table 4

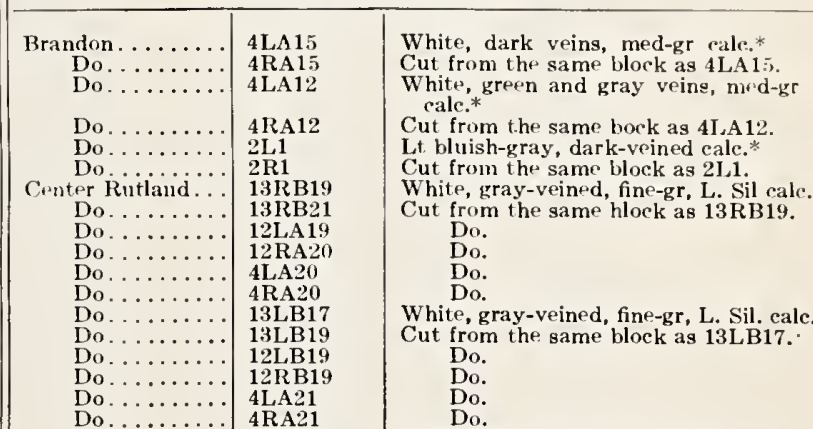

White, dark veins, med-gr calc.*

Cut from the same block as $4 \mathrm{LA} 13$. Do.

White, dark veins, med-gr calc, "Highland Gray Danby".*
Cut from the same block as $11 \mathrm{LB} 1$.

Cut from the same block as 11LB17.
White, med-gr, calc, "Imperial Yellow Danby":**

\begin{tabular}{c|c} 
Do................... 11RB17 \\
12LA18
\end{tabular}

Do........ 12RA19

Do........... $2 \mathrm{~L}^{\mathrm{L}}$

Do $\ldots \ldots \ldots \ldots 2$ R5

Do........... $3 \mathrm{~L} 1$

Do........ 3R1

\begin{tabular}{l|l} 
Do $\ldots \ldots \ldots \ldots$ & $3 \mathrm{R} 1$ \\
Do $\ldots \ldots$ & $3 \mathrm{~L} 3$
\end{tabular}

Do........ $3 \mathrm{R} 3$

Do........... 2 2I 8

Do........ $2 \mathrm{R} 8$

Do........... $2 \mathrm{~L} 7$

Do........ 2R7

Do...........

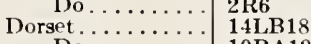

Dorset ift.......... 13RA17

F. Dorset....... 12RB3

Florence.......... $2 \mathrm{~L} 3$

Do......... 2R3

Do............ $2 \mathrm{~L} 12$

Do......... 2R12

Do.......... 2 2

Do........ 2R2

Cut from the same block as 12LA18.

White, yellow-clouded, med-gr, cale, "Imperial Danby Building and Memorial Grade".* Do.

White, dark-clouded, med-gr, calc, "Danby D Building Grade".

White, dark-veined, med-gr, calc "Danby D Building Grade"."* Do.

Lt bluish-gray, dark-veined, med-gr calc, "Danby Highland Building Grade".** Do

White, dark-veined, med-gr, calc, "Danby $J$ Building Grade and Riverside Danby Memorial Grade".* Do.

White, dark-veined, med-gr ealc.*

Cut from the same block as $2 \mathrm{~L} 6$.

Cut from the same block as
White, med-gr, L. Sil. calc.* Do.

White, med-gr, L. Sil. calc, "Imperial Danby".*

White, blue-veined, med-gr, mg L. Sil.*

It-gray dark-veined, med-gr, calc, "Sterling

Lt-gray, dark and white-clouded, med-gr calc, "Standard PV Memorial Grade".* Do.

Lt-gray, dark-clouded, med-gr cale, "Sterling PV Memorial Grade".* Do. 
TABLE 1. Source, classification, and description of domestic stones in south face of wall--Continued

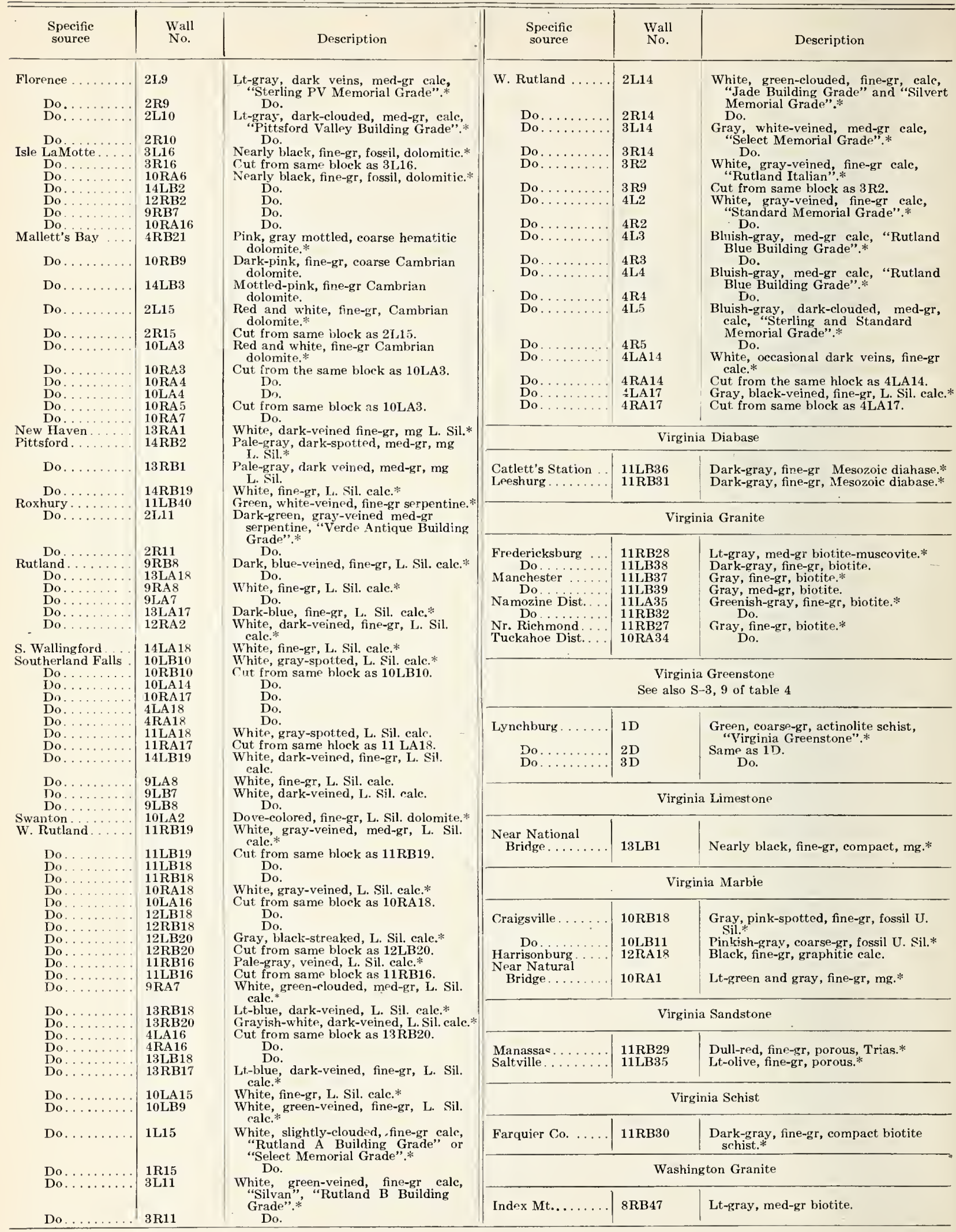


TABLE 1. Source, classification, and description of domestic stones in south face of wall-Continued

\begin{tabular}{|c|c|c|}
\hline $\begin{array}{l}\text { Specific } \\
\text { source }\end{array}$ & $\begin{array}{l}\text { Wall } \\
\text { No. }\end{array}$ & Description \\
\hline \multicolumn{3}{|c|}{ Washington Marble } \\
\hline $\begin{array}{l}\text { Lincoln Co. } \\
\text { Do.... } \\
\text { Stevens Co. }\end{array}$ & $\begin{array}{l}\text { 10LA1 } \\
\text { 10RA2 } \\
\text { 11RA2 }\end{array}$ & $\begin{array}{l}\text { White, med-gr dolomitic.* } \\
\text { Pale-pink, coarse-gr mg.* } \\
\text { Blue-gray, white-spotted, fine-gr, } \\
\text { dolomitic. }\end{array}$ \\
\hline \multicolumn{3}{|c|}{ Washington Sandstone } \\
\hline $\begin{array}{r}\text { Bellingham. } \\
\text { Do..... } \\
\text { Do.... } \\
\text { Do .... } \\
\text { Olymphia.. } \\
\text { Pittsburgh . } \\
\text { Tenino..... } \\
\text { Do.... } \\
\text { Willseson... }\end{array}$ & $\begin{array}{l}\text { 11RB 42 } \\
11 \mathrm{LB} 41 \\
\text { 8RA46 } \\
8 \mathrm{RA44} \\
\text { 8LA45 } \\
8 \mathrm{LB} 46 \\
8 \mathrm{RB} 46 \\
9 \mathrm{LB} 43 \\
8 \mathrm{LA46}\end{array}$ & $\begin{array}{l}\text { Gray, fine-gr, compact, "CChuckanut".*" } \\
\text { Gray, med-gr, porous, "Chuckanut.".* } \\
\text { Gray, fine-gr, porous, Carb.* } \\
\text { Gray, med-gr, compact, "Chuckanut".* } \\
\text { Dark-gray, med-gr, compact.* } \\
\text { Lt-gray, med-gr, compact, micaceous. } \\
\text { Lt-brown, coarse-gr, porous.* } \\
\text { Gray, med-gr, porous.** } \\
\text { Lt-gray, med-gr, compact calc.* }\end{array}$ \\
\hline
\end{tabular}

\section{West Virginia Marble}

\begin{tabular}{l|l|l}
\hline Harpers Ferry . . . & 8RB8 & Nearly black, yellow-vrined, fine-gr.
\end{tabular}

West Virginia Sandstone

\begin{tabular}{|c|c|c|}
\hline 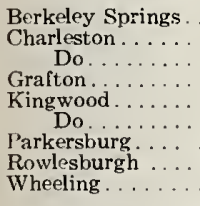 & $\begin{array}{l}\text { 10RB337 } \\
\text { 8RA399 } \\
\text { 9RB355 } \\
\text { 10LB37 } \\
\text { 9LA34 } \\
\text { 9RA36 } \\
\text { 8LA40 } \\
\text { 8LB40 } \\
\text { 9LB39 }\end{array}$ & $\begin{array}{l}\text { Dull-red, fine-gr, compact. } \\
\text { Gray, med-gr, compact Carb.* } \\
\text { Lt-buff, coarse-gr, porous Carb.* } \\
\text { Lt-buff, coarse-gr, porous Carb. } \\
\text { Buff, rust-spotted, med-gr, porous.* } \\
\text { Cut from same block as 9LA34. } \\
\text { Greenish-gray, med-gr, porous Carb.* } \\
\text { Dark-gray, fine-gr, compact Devonian.* } \\
\text { Gray, med-gr, porous Carb.* }\end{array}$ \\
\hline \multicolumn{3}{|c|}{ Wisconsin Dolomite } \\
\hline 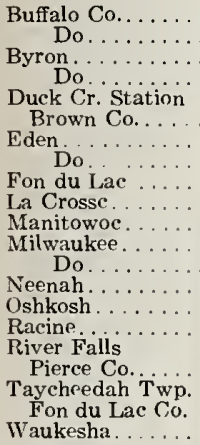 & $\begin{array}{l}\text { 11RB4 } \\
\text { 14RA5 } \\
\text { 10RA9 } \\
\text { 13RB4 } \\
\text { 12IA6 } \\
\text { 14RB5 } \\
\text { 14LA5 } \\
\text { 11LB2 } \\
\text { 13LA5 } \\
\text { 12RB6 } \\
\text { 12RA6 } \\
\text { 13RA4 } \\
\text { 14RA6 } \\
\text { 10LA5 } \\
\text { 13LB4 } \\
\text { 13LA4 } \\
\text { 14LA6 } \\
\text { 11LA3 }\end{array}$ & $\begin{array}{l}\text { Lt-buff, fine-gr, compact L. Sil.* } \\
\text { Yellowish-gray, fine-gr, porous L.. Sil.* } \\
\text { Lt-gray, fine-gr, compact. U. Sil.* } \\
\text { Lt-gray, fine-gr, compact U. Sil.* } \\
\text { Drab, fine-gr, compact L. Sil.* } \\
\text { Lt-drab, fine-gr U. Sil.* } \\
\text { Lt-gray, fine-gr compact.* } \\
\text { Lt-gray, fine-gr, compact U. Sil.* } \\
\text { Lt-huf,, fine-gr, porous L. Sil.* } \\
\text { Drab, fine-gr, compact U. Sil.* } \\
\text { Lt-drab, fine-gr, compact U. Sil.* } \\
\text { Drab, fine-gr, compact Sill.* } \\
\text { Dark-gray, fine-gr, compact L. Sil.* } \\
\quad \text { Do. } \\
\text { I.t-drab, fine-gr, cellular U. Sil.* } \\
\text { Buff, fine-gr, compact L. Sil.* } \\
\text { Lt-gray, fine-gr, compact U. Sil.* } \\
\text { Drab, fine-gr, compact U. Sil.* }\end{array}$ \\
\hline
\end{tabular}

Wisronsin Gneiss

\begin{tabular}{l|l|l}
\hline Black River Falls . & 8 LB45 & Reddish, fine-gr biotite. \\
\hline
\end{tabular}

\begin{tabular}{|c|c|c|}
\hline $\begin{array}{l}\text { Specific } \\
\text { source }\end{array}$ & $\begin{array}{l}\text { Wall } \\
\text { No. }\end{array}$ & Deseription \\
\hline \multicolumn{3}{|c|}{$\begin{array}{l}\text { Wisconsin Granite } \\
\text { See also S-2, } 82 \text { of tahle } 4\end{array}$} \\
\hline 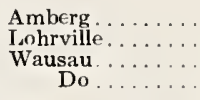 & $\begin{array}{l}\text { 7RB44 } \\
\text { 7RA43 } \\
\text { 8LB444 } \\
\text { 8LA44 }\end{array}$ & $\begin{array}{l}\text { Red and white, coarse-gr biotite.* } \\
\text { Pinkish-gray, fine-gr hiotite.* } \\
\text { Buff, fine-gr hiotite.* } \\
\text { Dark-red, fine-gr hornblende.* }\end{array}$ \\
\hline \multicolumn{3}{|c|}{ Wisconsin Limestrne } \\
\hline $\begin{array}{l}\text { Hayton .......... } \\
\text { Madison . Chien } \\
\text { Prairie du Chien }\end{array}$ & $\begin{array}{l}8 \mathrm{LB} 15 \\
8 \mathrm{RB} 16 \\
\text { 12LB29 }\end{array}$ & $\begin{array}{l}\text { Lt-drah, fine-gr, compact, cellular.* } \\
\text { Buff, fine-gr, porous L. Sil.** } \\
\text { Lt-buff, fine-gr, compact.** }\end{array}$ \\
\hline \multicolumn{3}{|c|}{ Wisconsin Quartzite } \\
\hline $\begin{array}{l}\text { Stevens Point. . . } \\
\text { Waterloo........ } \\
\text { Do ....... }\end{array}$ & $\begin{array}{l}\text { 7RB43 } \\
\text { 7LA43 } \\
\text { 6RA47 }\end{array}$ & $\begin{array}{l}\text { Lt-gray, fine-gr, compact. } \\
\text { Gray, coarse-gr.* } \\
\text { Gray, coarse-gr, compact paving } \\
\text { stone.* }\end{array}$ \\
\hline \multicolumn{3}{|c|}{ Wismonsin Quartz Porphyry } \\
\hline $\begin{array}{l}\text { Brandon ....... } \\
\text { Marquette.... }\end{array}$ & $\begin{array}{l}11 \mathrm{RB} 40 \\
10 \mathrm{RB} 49\end{array}$ & $\begin{array}{l}\text { Nearly black, coarse-gr, compact.* } \\
\text { Dark-gray, coarse-gr, compact.* }\end{array}$ \\
\hline \multicolumn{3}{|c|}{ Wisconsin Sandstone } \\
\hline 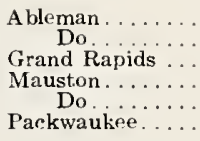 & $\begin{array}{l}\text { 9RB41 } \\
\text { 10RB48 } \\
\text { 9LB42 } \\
\text { 10LB47 } \\
\text { 10LB46 } \\
\text { 8RB45 }\end{array}$ & $\begin{array}{l}\text { L.t-buff, fine-gr, compact L. Sil. } \\
\text { Lt-buf, fine-gr, porous, friable L. Sil.* } \\
\text { Ltt-buff, fine-gr, compact L. Sil.** } \\
\text { Lt-red, fine-gr, porous L. Sil.** } \\
\text { Lt-gray, fine-gr, porous L. Sili.* } \\
\text { Lt-buff, med-gr, porous L. Sil.** }\end{array}$ \\
\hline \multicolumn{3}{|c|}{ Wyoming Diorite } \\
\hline Raw Hide Buttes & 9RA40 & Nearly black, fine-gr, compact. \\
\hline \multicolumn{3}{|c|}{ Wyoming Granite } \\
\hline 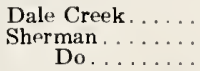 & $\begin{array}{l}\text { 10LA47 } \\
\text { 10RA42 } \\
\text { 10RA43 }\end{array}$ & $\begin{array}{l}\text { Red, coarse-gr hiotite* } \\
\text { Pink, fine-gr biotite. } \\
\text { Red, fine-gr biotite. }\end{array}$ \\
\hline
\end{tabular}

Wyoming Marble

\begin{tabular}{l|l|l}
\hline Wheatland ...... & 12LA12 & White, med-gr dolomitic.
\end{tabular}

Wyoming Sandstone

See also $\mathrm{S}-2,1, \mathrm{~S}-2,126$, and $26 \mathrm{WE}$ of tahle 4

\begin{tabular}{|c|c|c|}
\hline $\begin{array}{l}\text { Laramie. } \\
\text { Rawlins. }\end{array}$ & $\begin{array}{l}\text { 9LB44 } \\
\text { 9RA39 }\end{array}$ & $\begin{array}{l}\text { Lt-red, fine-gr, porous. } \\
\text { Lt-gray, fine-gr, porous, calk. }\end{array}$ \\
\hline
\end{tabular}


TABLE 2. Source, classification, and description of foreign stones in south face of wall

[For identification of samples in wall, see section 8 . Trade names are enclosed in quotation marks, geological terms are capitalized and a star $(*)$ at end of line signifies that the stone has been used commercially.]

\begin{tabular}{|c|c|}
\hline . & 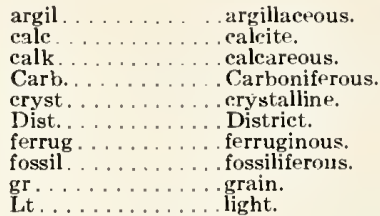 \\
\hline
\end{tabular}

ABBREVIATIONS

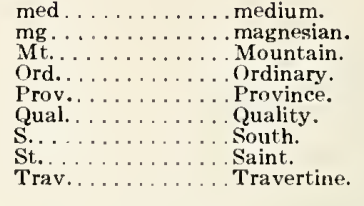

\begin{tabular}{|c|c|c|}
\hline $\begin{array}{l}\text { Specific } \\
\text { source }\end{array}$ & $\begin{array}{l}\text { Wall } \\
\text { No. }\end{array}$ & Description \\
\hline \multicolumn{3}{|c|}{ Argentine Building Stones } \\
\hline $\begin{array}{l}\text { Unknown . . . . . } \\
\text { Do . . . } \\
\text { Do . }\end{array}$ & $\begin{array}{l}\text { 10LB } 15 \\
10 \mathrm{RB} 16 \\
5 \mathrm{LB} 46\end{array}$ & $\begin{array}{l}\text { Lt-olive, brown-spotted marble. } \\
\text { Dark-gray, med-gr marble. } \\
\text { Dull-red and white, coarse-gr serpen- } \\
\text { tine. }\end{array}$ \\
\hline \multicolumn{3}{|c|}{ Australian Granite } \\
\hline New South Wales & $1 \mathrm{R} 6$ & Black and white, coarse-gr hiotite. \\
\hline \multicolumn{3}{|c|}{ Australian Marble } \\
\hline 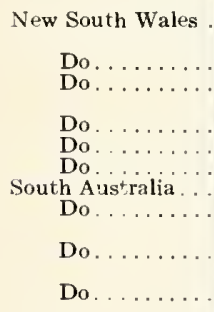 & $\begin{array}{l}3 \mathrm{~L} 10 \\
3 \mathrm{R} 10 \\
3 \mathrm{~L} 2 \\
7 \mathrm{RA3} \\
\text { 14RA19 } \\
\text { 14LA19 } \\
\text { 10LA17 } \\
\text { 7RA2 } \\
\text { 14RA20 } \\
\text { 7LA1 }\end{array}$ & $\begin{array}{l}\text { Gray, yellow and white-mottled, med-gr } \\
\text { cale. } \\
\text { Cut from the same block as } 3 \mathrm{~L} 10 \text {. } \\
\text { White, greenish and yellow-veined, } \\
\text { med-gr calc. } \\
\text { Gray-variegated, fine-gr ealc. } \\
\text { Grayish-white, fine-gr. } \\
\text { Black, fine-gr, fossil calc. } \\
\text { Gray, med-gr calc. } \\
\text { Brownish-white with brown veins, } \\
\text { med-gr. } \\
\text { Gray and white-mottled and veined, } \\
\text { med-gr. } \\
\text { Buff and gray-mottled, med-gr. }\end{array}$ \\
\hline
\end{tabular}

Australian Sandstone

\begin{tabular}{|c|c|c|}
\hline 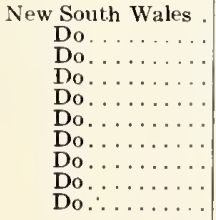 & $\begin{array}{l}\text { 4L7 } \\
4 \mathrm{R} 7 \\
9 \mathrm{LB} 26 \\
\text { 9RA26 } \\
\text { 9LA27 } \\
\text { 9LB27 } \\
\text { 9RB24 } \\
\text { 9RB25 } \\
\text { 4L8 } \\
4 \mathrm{R} 8\end{array}$ & $\begin{array}{l}\text { Pinkish-buff, med-gr, porous. } \\
\text { Cut from the same block as } 4 \mathrm{~L} 7 . \\
\text { Pinkish-buff, coarse-gr, porous. } \\
\text { Cut from the same block as } 9 \text { LB26. } \\
\text { Do. } \\
\text { Do. } \\
\text { Do. } \\
\text { Do. } \\
\text { Lt-buff, coarse-gr, porous. } \\
\text { Cut from the same block as } 4 \mathrm{~L} 8 \text {. }\end{array}$ \\
\hline
\end{tabular}

Austrian Conglomerate

Lindabrunn ..... 5 LB27 $\mid$ Lt-yellow, coarse-gr, porous, calk.

Austrian Granite

\begin{tabular}{l|l|l}
\hline $\begin{array}{l}\text { Crasstein. . . . . . } \\
\text { Neuhaus...... }\end{array}$ & $\begin{array}{l}\text { 5RB29 } \\
5 \mathrm{LB} 26\end{array}$ & $\begin{array}{l}\text { Black and white, med-gr, hiotite. } \\
\text { Grav, fine-gr, biotite granite. }\end{array}$ \\
\hline
\end{tabular}

Austrian Limestone

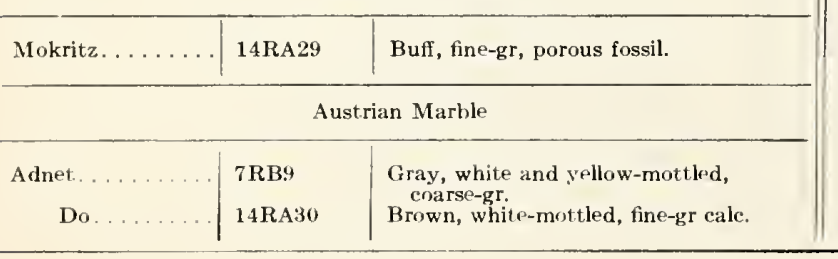

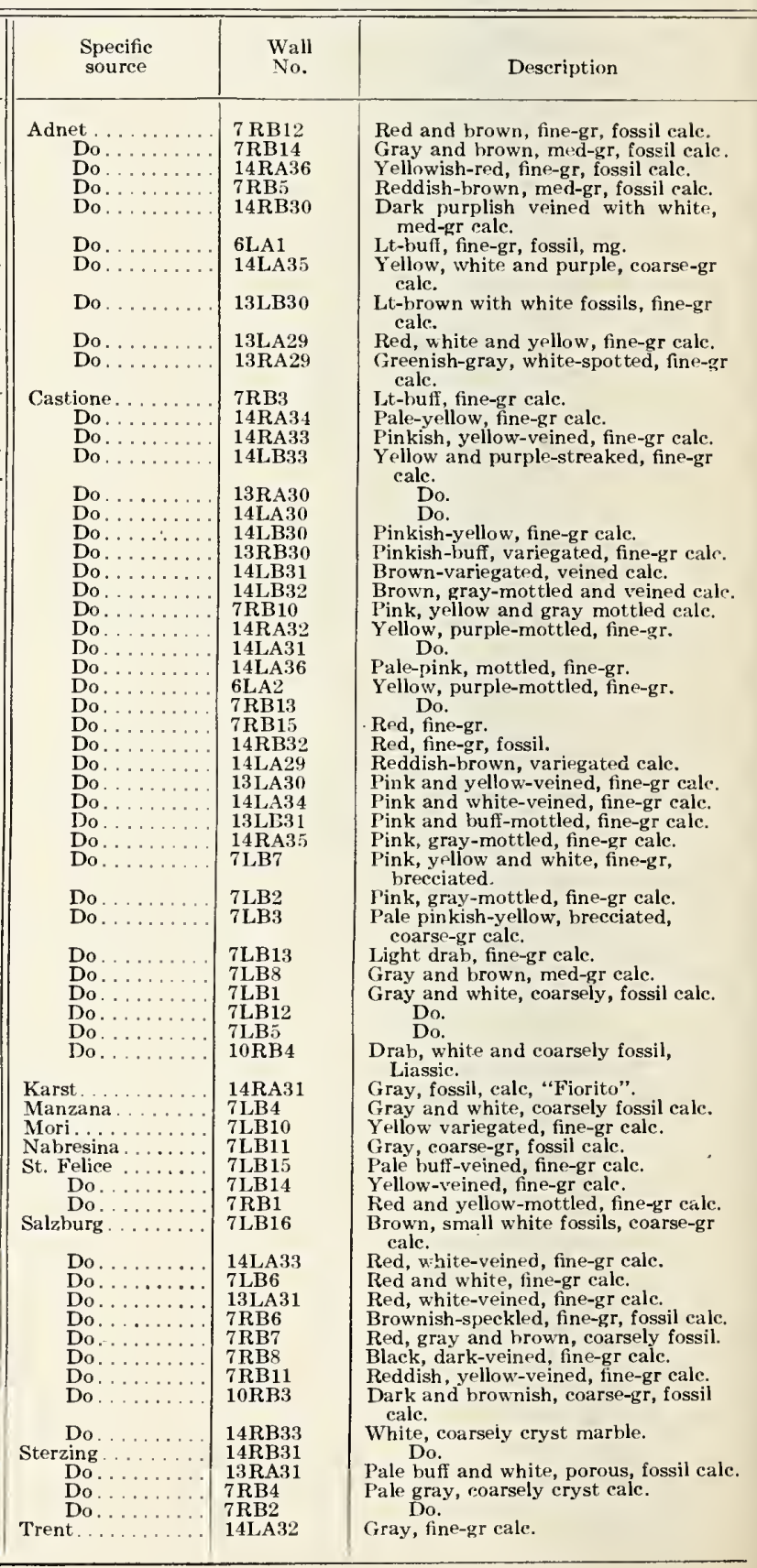


TABLE 2. Source, classification, and description of foreign stones in south face of wall-Continued

\begin{tabular}{|c|c|c|}
\hline $\begin{array}{l}\text { Specific } \\
\text { source }\end{array}$ & $\begin{array}{l}\text { Wall } \\
\text { No. }\end{array}$ & Description \\
\hline \multicolumn{3}{|c|}{ Austrian Pitchstone } \\
\hline $\begin{array}{l}\text { Castleruth . . . . . } \\
\text { Do . . . . . }\end{array}$ & $\begin{array}{l}\text { 5RA28 } \\
\text { 5LA26 }\end{array}$ & $\begin{array}{l}\text { Dark-brown, coarse-gr. } \\
\text { Dark-gray, spotted with white, } \\
\text { coarse-gr. }\end{array}$ \\
\hline \multicolumn{3}{|c|}{ Austrian Quartz Porphyry } \\
\hline $\begin{array}{c}\text { South Tyrol . . . . . } \\
\text { Do....... }\end{array}$ & $\begin{array}{l}\text { 5RB28 } \\
\text { 5LA27 }\end{array}$ & $\begin{array}{l}\text { Reddish, white-spotted, med-gr } \\
\text { Red, spotted with white, med-g }\end{array}$ \\
\hline
\end{tabular}

Austria-Hungarian Marble

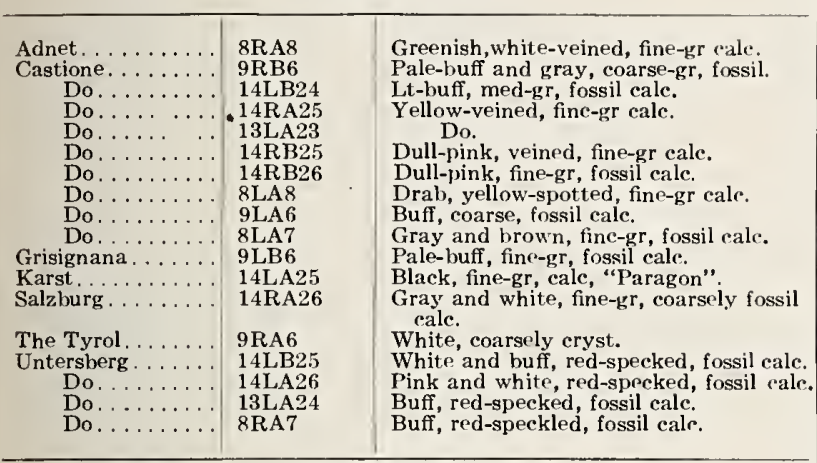

Bermuda Limestone

\begin{tabular}{c|l|l}
\hline $\begin{array}{c}\text { Coralline } \ldots \ldots \ldots \\
\text { Do. . . . }\end{array}$ & $\begin{array}{l}\text { 8LB9 } \\
8 R B 10\end{array}$ & $\begin{array}{l}\text { Nearly white, coarsely cellular, friable. } \\
\text { Cut from the same block as 8LB9. }\end{array}$
\end{tabular}

Canadian Granite

\begin{tabular}{r|l|l}
\hline Halifax Co. ..... & $1 \mathrm{L6}$ & $\begin{array}{l}\text { Gray, coarse-gr biotite-muscovite, } \\
\text { porphyritic. } \\
\text { Bright-red, coarse-gr hornblende. } \\
\text { Lt-red, coarse-gr biotite granite. }\end{array}$ \\
\hline
\end{tabular}

Canadian Limestone

\begin{tabular}{l|l|l}
\hline $\begin{array}{l}\text { Kingston . . . . . . } \\
\text { Montreal . . . }\end{array}$ & $\begin{array}{l}\text { 8LA9 } \\
\text { 8RA9 . . . . }\end{array}$ & $\begin{array}{l}\text { Dark-gray, fine-gr, compact, fossil. } \\
\text { Dark-gray, med-gr, compact, semi- } \\
\text { cryst. } \\
\text { Dark-gray, fine-gr, compact. }\end{array}$ \\
\hline
\end{tabular}

Canadian Marble

\begin{tabular}{|c|c|c|}
\hline Phillipsburg . & 13LB24 & Grayish-white, fine-gr calc. \\
\hline 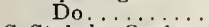 & $13 \mathrm{LB} 25$ & Cut from same block as $131 . \mathrm{B} 24$. \\
\hline S. Stukely, Quebec & $4 \mathrm{RB} 19$ & $\begin{array}{l}\text { Pink and white variegated, med-gr } \\
\text { calc. }\end{array}$ \\
\hline $\begin{array}{l}\text { Stony Mt., Near } \\
\text { Winnipeg. . . . . . }\end{array}$ & 12I.A23 & Purple and buff, fossil calc. \\
\hline
\end{tabular}

Canadian Sandstone

Brampton, Ontario
Cliffton,
New Brunswick.
Do..........
Dorchester,
New Brunswick.
Georgetown,
Ontario........
Mary's Point,
New Brunswick.
Nepigon Bay,
Ontario........
Sackville,
New Brunswick.

\begin{tabular}{|l|l} 
9LA46 & Brown, fine-gr, compact.* \\
11RB46 & $\begin{array}{l}\text { Greenish-gray, med-gr, compact. } \\
\text { 11LB43 }\end{array}$ \\
9RA45 & Olive, fine-gr, porous, Sub-Carb. \\
9RA46 & Light-gray, fine-gr, porolas. \\
9LA45 & Brown, fine-gr, compact, Carb. \\
9RB43 & Red, fine-gr, compact, Potsdam.* \\
10LA50 & Brown, med-gr, porous.*
\end{tabular}

\begin{tabular}{|c|c|c|}
\hline $\begin{array}{l}\text { Specific } \\
\text { source }\end{array}$ & $\begin{array}{l}\text { Wall } \\
\text { No. }\end{array}$ & Description \\
\hline \multicolumn{3}{|c|}{ Cuhan Marhle } \\
\hline 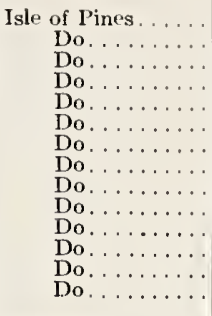 & $\begin{array}{l}8 \mathrm{LB} 10 \\
8 \mathrm{LB} 11 \\
8 \mathrm{RB} 11 \\
8 \mathrm{RB} 12 \\
10 \mathrm{LA} 23 \\
\text { 10RA25 } \\
10 \mathrm{RB11} \\
11 \mathrm{LA} 22 \\
11 \mathrm{RB} 23 \\
11 \mathrm{RA2} 2 \\
13 \mathrm{LA} 22 \\
13 \mathrm{LB} 23 \\
\text { 14RB27 } \\
\text { 13RA25 }\end{array}$ & $\begin{array}{l}\text { Gray and dark-streaked, coarse-gr calc. } \\
\text { Gray and dark-streaked, coarse-gr calc. } \\
\text { Bluish-gray, coarse-gr calc. } \\
\text { Bluish-gray, dark-veincd, coarse-gr calc. } \\
\text { White, fine-gr, dolomitic. } \\
\text { Lt bluish-gray, med-gr, dolomitic. } \\
\text { Dark blnish-gray, coarse-gr ealc. } \\
\text { Blaish-gray, coarse-gr calc. } \\
\text { Lt-bluish-gray, med-gr dolomitic. } \\
\text { Bluish-gray, coarse-gr calc. } \\
\text { Bluish-gray, med-gr cale. } \\
\text { Bluish-gray, coarse-gr calc. } \\
\text { Lt-bluish-gray, coarse-gr calc. } \\
\text { Lt-gray, coarse-gr calc. }\end{array}$ \\
\hline \multicolumn{3}{|c|}{ English Marble } \\
\hline $\begin{array}{r}\text { Bristol } \ldots \ldots \ldots \\
\text { Do } \ldots . . . . . .\end{array}$ & $\begin{array}{l}\text { 10LB14 } \\
\text { 10RB15 }\end{array}$ & $\begin{array}{l}\text { Gray and brown, fine-gr dendritic. } \\
\text { Cut from the same blnek as 10LB14. }\end{array}$ \\
\hline \multicolumn{3}{|c|}{ English Serpentine } \\
\hline $\begin{array}{l}\text { Cadgrwith Dist. } \\
\text { Cornwall... }\end{array}$ & 10LA36 & Olive-green, red-veined. \\
\hline
\end{tabular}

Finland Granite

Sre sample S-2, 75 of table 4

Hungarian Conglomerate

\begin{tabular}{l|l|l}
\hline $\begin{array}{l}\text { Hundscheim. ..... } \\
\text { Leitha Mts. } \\
\text { Sommercin ....... }\end{array}$ & $\begin{array}{l}\text { 5RB45 } \\
\text { 5LB45 } \\
\text { 5RA45 }\end{array}$ & $\begin{array}{l}\text { Buff, drab-spotted, conrse-gr, calk. } \\
\text { Grayish, coarse-gr, porous calk. } \\
\text { Pale-buff, fine-gr, porous calk. }\end{array}$
\end{tabular}

Hungarian Limestone

\begin{tabular}{|c|c|c|}
\hline $\begin{array}{l}\text { Breiten brunn . . . . } \\
\text { Kroisbach........ } \\
\text { Lcitha Mts. } \\
\text { Do........ } \\
\text { Mannersdorf .... }\end{array}$ & $\begin{array}{l}\text { 13RA36 } \\
14 \text { RA37 } \\
\text { 10LB8 } \\
7 \text { RA6 } \\
7 \text { RA7 }\end{array}$ & $\begin{array}{l}\text { Ltt-buff, fine-gr, porous. } \\
\text { Nearly white, med-gr, porous. } \\
\text { Lt-buff, coarse-gr, cellular, fossil. } \\
\text { Lt-buff, coarse-gr, cellular, fossil. } \\
\text { Pale-buff and white, coarse-gr, porous, } \\
\text { fossil. }\end{array}$ \\
\hline 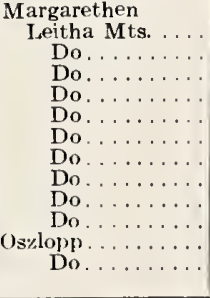 & $\begin{array}{l}\text { 14LA37 } \\
\text { 13LA36 } \\
\text { 14RB37 } \\
\text { 7RA5 } \\
\text { 14RA38 } \\
\text { 14LB37 } \\
\text { 7LA5 } \\
\text { 7LA4 } \\
\text { 7LB9 } \\
\text { 7LA6 } \\
\text { 7LA3 } \\
\text { 14LA38 }\end{array}$ & $\begin{array}{l}\text { Lt-buff fine-gr, porous. } \\
\text { Lt-buff, fine-gr, porous. } \\
\text { Lt-buff, med-gr, cellular, fossil. } \\
\text { Buff, med-gr, porous, fossil. } \\
\text { Buff, med-gr, porous, fossil. } \\
\text { Lt-liuff coarse-gr, porous, fossil. } \\
\text { Lt-buff, med-gr, cellular, fossil. } \\
\text { Yellow, coarse-gr, porols, fossil. } \\
\text { Yellow, coarse-gr, porous, fossil. } \\
\text { Buff, coarse-gr, cellular, fossil. } \\
\text { Buff-variegated, med-gr, compact, fossil. } \\
\text { Buff and yellow, coarse-gr, porous, } \\
\text { fossil. }\end{array}$ \\
\hline
\end{tabular}

Hunzarian Marble

\begin{tabular}{l|l|l} 
Mannersdorf ..... & 7RA4 & Pale-buff and white, fossil calc.
\end{tabular}

Italian Limestone

\begin{tabular}{c|c|c}
\hline $\begin{array}{c}\text { Leghorn } \ldots \ldots \ldots \ldots \\
\text { Do . . . . }\end{array}$ & $\begin{array}{l}\text { 4LB19 } \\
\text { 13LB29 }\end{array}$ & $\begin{array}{l}\text { Buff, fine-gr, compact travertine.* } \\
\text { Dull-buff, fine-gr, porous. }\end{array}$
\end{tabular}

Italian Granite

See section S-1 of table 4

\begin{tabular}{|c|c|c|}
\hline \multicolumn{3}{|c|}{ Italian Marble } \\
\hline $\begin{array}{l}\text { Carrara } \ldots \ldots \ldots \\
\text { Do } \ldots \ldots \ldots \\
\text { Do } \ldots \ldots \ldots \\
\text { Do } \ldots \ldots \ldots \\
\text { Do. } \ldots \ldots \ldots \\
\text { Do } \ldots \ldots \\
\text { Do } \ldots \ldots\end{array}$ & $\begin{array}{l}\text { 2L16 } \\
\text { 2R16 } \\
3 \mathrm{~L} 6 \\
\text { 3R6 } \\
\text { 3L9 } \\
\text { 12LB27 } \\
\text { 12RB28 }\end{array}$ & $\begin{array}{l}\text { White, fine-gr calc.* } \\
\text { Cut from the same block as } 2 \mathrm{~L} 16 . \\
\text { White, pale-gray clouds, fine-gr calc.* } \\
\text { Cut from the same block as 3L } 6 \text {. } \\
\text { Yellowish-white, fine-gr, statuary calc.* } \\
\text { Cut from the same block as 3L9. } \\
\text { Do. }\end{array}$ \\
\hline
\end{tabular}


TABLE 2. Source, classification, and description of foreign stones in south face of wall-Continued

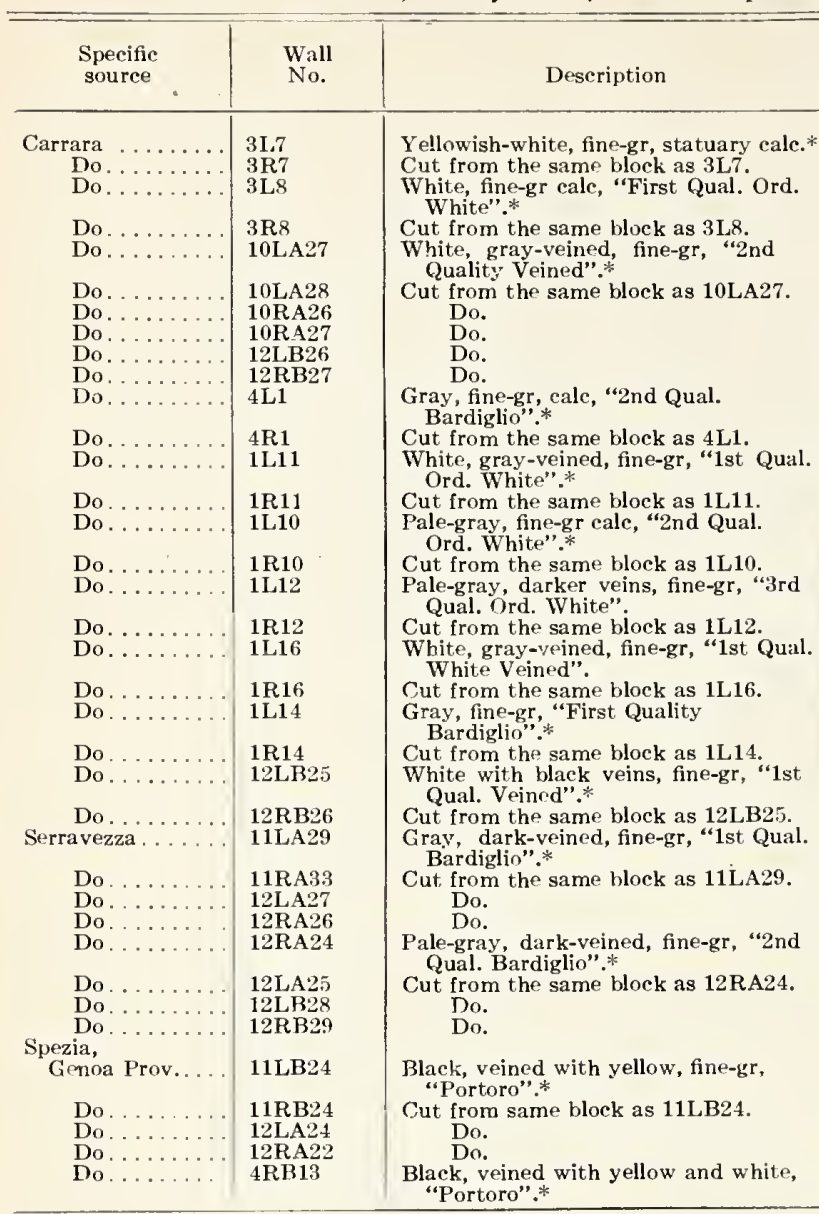

Italian Sandstone

\begin{tabular}{r|l|l}
$\begin{array}{r}\text { Leghorn . . . . . } \\
\text { Do . . . . }\end{array}$ & $\begin{array}{l}\text { 9RA32 } \\
\text { 11LB25 }\end{array}$ & $\begin{array}{l}\text { Gray, coarse-gr, compact, calk.* } \\
\text { Cut from same block as 9RA32. }\end{array}$
\end{tabular}

Italian Travertine

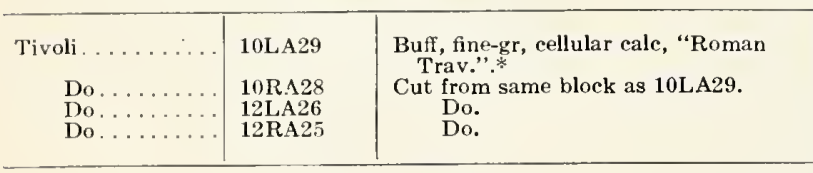

Mexican Andesite

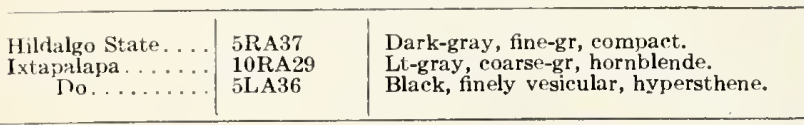

Mexican Basalt

\begin{tabular}{r|l|l}
$\begin{array}{c}\text { Mexico State.... } \\
\text { Puebla State .... }\end{array}$ & $\begin{array}{l}\text { 10RB34 } \\
\text { 5RB37 } \\
\text { Do......... }\end{array}$ & $\begin{array}{l}\text { Dull-red, very vesicular. } \\
\text { Nearly black, fine-gr, coarsely } \\
\text { vesicular. } \\
\text { Cut from the same block as 5RB37. }\end{array}$
\end{tabular}

Mexican Dacite

Mexico State.... $\mid$ 10LA38 $\mid$ Reddish-brown, fine-gr, porous.

\begin{tabular}{|c|c|c|}
\hline $\begin{array}{l}\text { Specific } \\
\text { source }\end{array}$ & $\begin{array}{l}\text { Wall } \\
\text { No. }\end{array}$ & Description \\
\hline \multicolumn{3}{|c|}{ Mexican Pumice } \\
\hline $\begin{array}{l}\text { San Juan, } \\
\text { Pueblo State ... }\end{array}$ & 10RB35 & Gray, fine-gr, coarsely vesicular. \\
\hline \multicolumn{3}{|c|}{ Mexican Pyrophyllite } \\
\hline $\begin{array}{r}\text { Sonora State } \ldots \\
\text { Do. . . . . . } \\
\text { Do . . }\end{array}$ & $\begin{array}{l}\text { 9LB33 } \\
\text { 9RB31 } \\
\text { 10LB39 }\end{array}$ & $\begin{array}{l}\text { Lt-gray, buff-syotted, fine-gr. } \\
\text { Cut from same block as 9LB } 33 \text {. } \\
\text { Do. }\end{array}$ \\
\hline \multicolumn{3}{|c|}{ Mexican Sandstone } \\
\hline $\begin{array}{l}\text { Guanajuato State } \\
\text { Do......... }\end{array}$ & $\begin{array}{l}\text { 10LA39 } \\
\text { 10RA30 }\end{array}$ & $\begin{array}{l}\text { Green, brown-banded, med-gr, com- } \\
\text { pact, calk. } \\
\text { Green, brown-banded, med-gr, com- } \\
\text { ract, calk. }\end{array}$ \\
\hline
\end{tabular}

Mexican Tuff

\begin{tabular}{|c|c|c|}
\hline $\begin{array}{l}\text { Huehutoca ...... } \\
\text { Morelos State ... } \\
\text { Oaxaca State.... } \\
\text { Oaxaca State.... } \\
\text { Zacatecas State. . } \\
\text { Do. . . . . . . . } \\
\text { Do. . . . }\end{array}$ & $\begin{array}{l}\text { 6RB38 } \\
6 \mathrm{LB} 38 \\
6 \mathrm{LA3} 3 \\
6 \mathrm{RB} 37 \\
\text { 10LA40 } \\
\text { 6RA37 } \\
\text { 10LA41 }\end{array}$ & $\begin{array}{l}\text { Red, coarse-gr, rhyolite. } \\
\text { Lt reddish-brown, fine-gr, compact. } \\
\text { Lt-green, fine-gr, compact, rhyolite. } \\
\text { Brown, white-mottled, fine-gr, porous. } \\
\text { Buff, brown-banded, fine-gr, compact. } \\
\text { Pinkish, fine-gr, compact, rhyolite. } \\
\text { Greenish-gray, fine-gr, compact, } \\
\text { rhyolite. }\end{array}$ \\
\hline
\end{tabular}

Portuguese Limestone

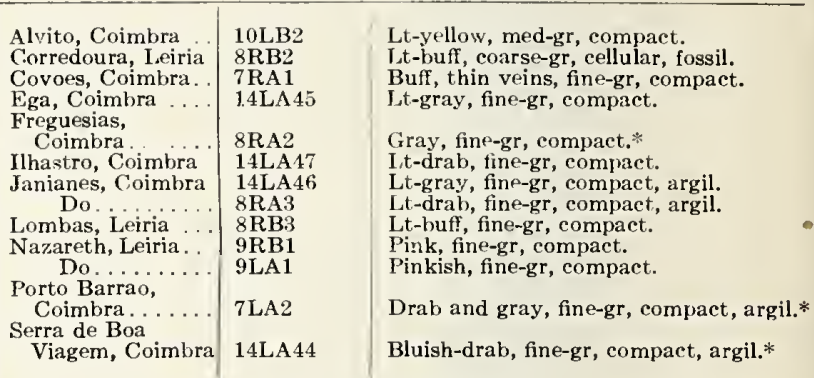

Portuguese Marble

\begin{tabular}{|c|c|c|}
\hline Alvrite, Coimbra. . & 8LB1 & Gray, med-gr, calc. \\
\hline Ameixoira, & 8T A? & It-aray fine-gr \\
\hline $\begin{array}{l}\text { Colmbra........ } \\
\text { Andorinha, }\end{array}$ & 8LA2 & Lt-gray, fine-gr. \\
\hline Coimbra...... & 8LA1 & Lt-yellow, fine-gr. \\
\hline $\begin{array}{l}\text { Arrabida Mts., } \\
\text { Lisbon Dist... . . }\end{array}$ & 10LB1 & $\begin{array}{l}\text { Red and yellow, coarse-gr calk } \\
\text { conglomerate. }\end{array}$ \\
\hline Do.. & 10RB1 & Cut from the same block as 10LB1. \\
\hline $\begin{array}{l}\text { Cintra, } \\
\text { Lisbon Dist..... }\end{array}$ & 10RB2 & Yellow, firte-gr. \\
\hline $\begin{array}{l}\text { Condeixa a } \\
\text { Velha, Coimbra }\end{array}$ & $8 \mathrm{LA} 3$ & Lt pinkish-drab, fine-gr. \\
\hline 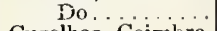 & 8LB3 & Pink and drab-mottled, fine-gr. \\
\hline $\begin{array}{l}\text { Cuzelhas, Coimbra } \\
\text { Estremoz. }\end{array}$ & $8 \mathrm{LB} 2$ & Lt-yellow, fine-gr. \\
\hline $\begin{array}{l}\text { Alemtejo Prov. } \\
\text { Farrestello, }\end{array}$ & 12RB37 & IVhite, yellow-seined, med-gr calc.* \\
\hline $\begin{array}{l}\text { Figueira da Foz. } \\
\text { Figueira Zameirao, }\end{array}$ & 14RA43 & Pink and yellowish fine-gr fossil calc. \\
\hline $\begin{array}{l}\text { Coimbra . } \\
\text { Freguesias of } \mathrm{s} \text {. }\end{array}$ & 9LB1 & White, dark-spotted, fine-gr. \\
\hline $\begin{array}{l}\text { Freguesias of } \\
\text { Miguel, Coimbra }\end{array}$ & 14RA 40 & Yellow, purple-striped, fine-gr.* \\
\hline Do........... & $14 \mathrm{LA} 40$ & Dull-pink, fine-gr. ${ }^{*}$ \\
\hline $\begin{array}{l}\text { Do } \ldots \ldots \ldots \\
\text { Do............ }\end{array}$ & $\begin{array}{l}\text { 14LA } 43 \\
8 \mathrm{RB} 1\end{array}$ & $\begin{array}{l}\text { Dull-red, fine-gr." } \\
\text { Drab, pink-tinted, fine-gr.* }\end{array}$ \\
\hline Do............... & 14LA39 & White, red-spotted, fine-gr.* \\
\hline Do..... & 14RA44 & Dull-red, lt-streaked, fine-gr.* \\
\hline $\begin{array}{l}\text { Do } \ldots . . . \\
\text { Do..... }\end{array}$ & $\begin{array}{l}\text { 14RA42 } \\
\text { 9RA1 }\end{array}$ & $\begin{array}{l}\text { Lt-pink, drab-veined, fine-gr.** } \\
\text { Lt-pink, fine-gr.** }\end{array}$ \\
\hline Do..... & 14RA41 & $\begin{array}{l}\text { Drab, pink and yellow-streaked, } \\
\text { fine-gr.* }\end{array}$ \\
\hline Do. & 14RA39 & Lt-drab, fine-gr.* \\
\hline
\end{tabular}


TABLE 2. Source, classification, and description of foreign stones in south face of wall-Continued

\begin{tabular}{|c|c|c|c|c|c|}
\hline $\begin{array}{l}\text { Specific } \\
\text { source }\end{array}$ & $\begin{array}{l}\text { Wall } \\
\text { No. }\end{array}$ & Description & $\begin{array}{l}\text { Specific } \\
\text { source }\end{array}$ & $\begin{array}{l}\text { Wall } \\
\text { No. }\end{array}$ & Description \\
\hline \multirow[b]{2}{*}{$\begin{array}{l}\text { Manoel Zuarte, } \\
\text { Leiria .......... } \\
\text { Penella, } \\
\text { Beira Prov. ..... } \\
\text { Ponde do Espinal, } \\
\text { Coimbra....... } \\
\text { Salmanha, } \\
\text { Coimora..... } \\
\text { Serrada, do Lager, } \\
\text { I.eiria Prov..... } \\
\text { Vianna do } \\
\text { Alemtejo........ }\end{array}$} & \multirow[b]{2}{*}{$\begin{array}{l}\text { 14LA42 } \\
14 \mathrm{RA} 47 \\
8 \mathrm{RA} 1 \\
14 \mathrm{RA} 46 \\
\text { 14RA45 } \\
\text { 14LA41 }\end{array}$} & \multirow[b]{2}{*}{$\begin{array}{l}\text { Dull-red, fine-gr.* } \\
\text { Lt-drab, red dish-veined, fine-gr calc. } \\
\text { Lt-gray, fine-gr, cellular. } \\
\text { Pink, fine-gr. } \\
\text { Dull-pink, fine-gr.* } \\
\text { White, dark-veined, med-gr calc. }\end{array}$} & \multicolumn{3}{|c|}{ Spanish Limestone } \\
\hline & & & 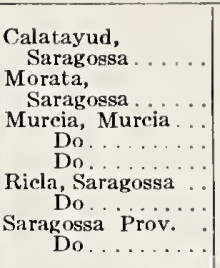 & $\begin{array}{l}\text { 14RB35 } \\
\text { 14LB35 } \\
\text { 13RA35 } \\
\text { 14RB36 } \\
\text { 9LA14 } \\
\text { 13LA35 } \\
\text { 10RB17 } \\
\text { 14RB34 } \\
\text { 9RA14 }\end{array}$ & $\begin{array}{l}\text { Dark-gray, fine-gr, compact. } \\
\text { Pinkish, fine-gr, compact. } \\
\text { Yellow, coarse-gr, porous, fossil. } \\
\text { l.t-gray, coarse-gr, friable. } \\
\text { Dark-gray, fine-gr, compact. } \\
\text { Dark-gray, fine-gr, compact. } \\
\text { Blue-black, fine-gr, compact. } \\
\text { Bluish-drab, fine-gr, compact. } \\
\text { Lt-drab, fine-gr, compact. }\end{array}$ \\
\hline \multicolumn{3}{|c|}{ Portuguese Sandstone } & \multicolumn{3}{|c|}{ Spanish Marble } \\
\hline $\begin{array}{l}\text { Sit. Miguel, } \\
\text { Coimbra ....... } \\
\text { Do........ } \\
\text { Do.... }\end{array}$ & $\begin{array}{l}\text { 11LA36 } \\
\text { 11LA37 } \\
\text { 9RB42 }\end{array}$ & $\begin{array}{l}\text { Red, fine-gr, compact, ferrug.* } \\
\text { Red, fine-gr, compact, calk. } \\
\text { Buff, coarse-gr, compact, calk. }\end{array}$ & $\begin{array}{l}\text { Cehegin, Murcia .. } \\
\text { Chodes, Saragossa. } \\
\text { Morata, Saragossa } \\
\text { Murcia, Murcia... } \\
\text { Panticosa, Huesca. } \\
\text { Puebla de Alborton } \\
\text { Ricla, Saragossa ... } \\
\text { Do ......... }\end{array}$ & $\begin{array}{l}\text { 13LB34 } \\
\text { 13LB35 } \\
\text { 13LA32 } \\
\text { 13LA33 } \\
\text { 13LA34 } \\
\text { 13RB32 } \\
\text { 13RA34 } \\
\text { 13RA32 }\end{array}$ & $\begin{array}{l}\text { Brown, yellow-mottled, fine-gr, calc. } \\
\text { Black and brown, coarse-gr breccia. } \\
\text { Dark and brown coarse-gr breceia. } \\
\text { Drab, buff-veined, fine-gr calc. } \\
\text { Dark blue-gray, med-gr calc. } \\
\text { Pink and white, cellular, breciated calc. } \\
\text { Black and white, coarse-gr, breciated. } \\
\text { Pinkish-purple, brown-spotted, fine-gr } \\
\text { calc. }\end{array}$ \\
\hline $\begin{array}{l}\text { Anan } \ldots . . . \ldots \\
\text { Ballochmyle...... } \\
\text { Beauty, Rose..... } \\
\text { Elgin, Moray ..... }\end{array}$ & $\begin{array}{l}\text { 5LA12 } \\
\text { 11LB28 } \\
\text { 7LA35 } \\
\text { 7 RA35 }\end{array}$ & $\begin{array}{l}\text { Dull-red, fine-gr, compact, Devonian. } \\
\text { I,t-red, fine-gr, porous, Carb. } \\
\text { Lt-red, fine-gr, compact, calls. } \\
\text { Buff, coarse-gr, compact. }\end{array}$ & $\begin{array}{l}\text { Do. } \ldots \ldots \ldots \\
\text { Do. } \ldots \ldots \ldots \\
\text { Do. } \ldots \ldots\end{array}$ & $\begin{array}{l}\text { 9RA15 } \\
\text { 13RA33 } \\
\text { 14LB34 }\end{array}$ & $\begin{array}{l}\text { Lt-buff, pink-veined, fine-gr calc. } \\
\text { Yellow and drab, coarse-gr calc. } \\
\text { Dark-drab, red veined, fine-gr calc. }\end{array}$ \\
\hline Thairn .............. & $\begin{array}{l}\text { 8RB36 } \\
\text { 8LB36 }\end{array}$ & $\begin{array}{l}\text { Lt-gr, finc-gr, porous, calk, Devonian. } \\
\text { Buff, med-gr, porons, calk, Devonian. }\end{array}$ & \multicolumn{3}{|c|}{ Spanish Sandstone } \\
\hline \multirow{2}{*}{\multicolumn{3}{|c|}{ Siamese Marble }} & Murcia......... & 9RA37 & Buff, fine-gr, compact, calk. \\
\hline & & & \multicolumn{3}{|c|}{ Spanish Tuff } \\
\hline$? \ldots \ldots \ldots \ldots \ldots$ & 9LA15 & Gray, white and yellow, coarse-gr, calc. & Alamazanon ...... & 9RA38 & Buff, med-gr, compact, cellular, calk. \\
\hline
\end{tabular}


TABLE 3. Course list for identifying samples, by position, in wall

[For explanation of use, see section 8.2.]

Abrreviations

\begin{tabular}{|c|c|c|}
\hline $\begin{array}{l}\text { Cong.. } \\
\text { D.M... } \\
\text { DM.... } \\
\text { Gr.... } \\
\text { Labr... }\end{array}$ & $\begin{array}{l}\text { conglomerate. } \\
\text { dolomite. } \\
\text { dolomitic marble. } \\
\text { gneiss. } \\
\text { granite. } \\
\text { labradorite. } \\
\text { limestone. } \\
\text { marble. }\end{array}$ & 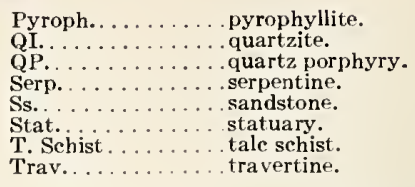 \\
\hline
\end{tabular}

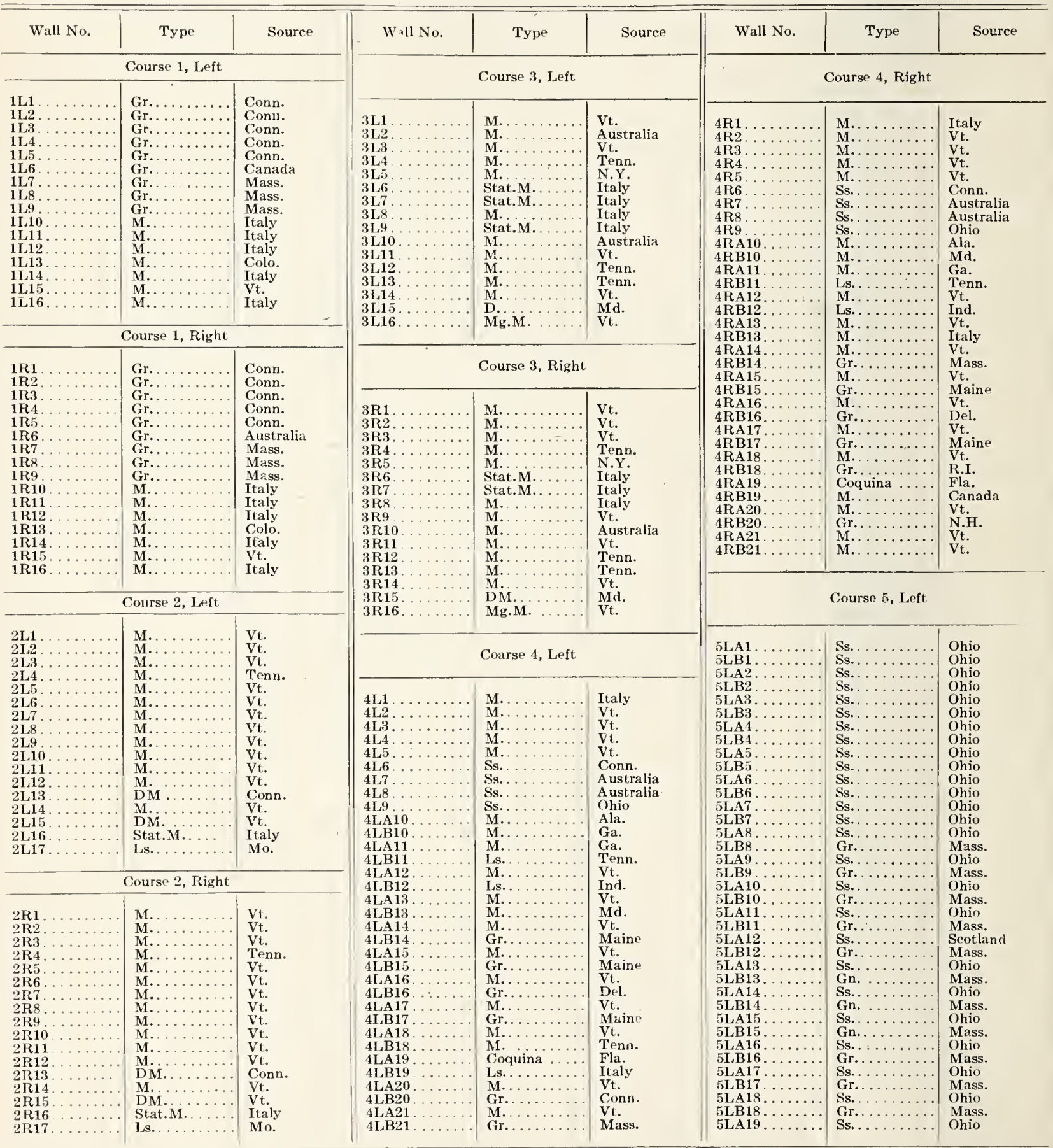


TABLE 3. Course list for identifying samples, by position, in wall-Continued

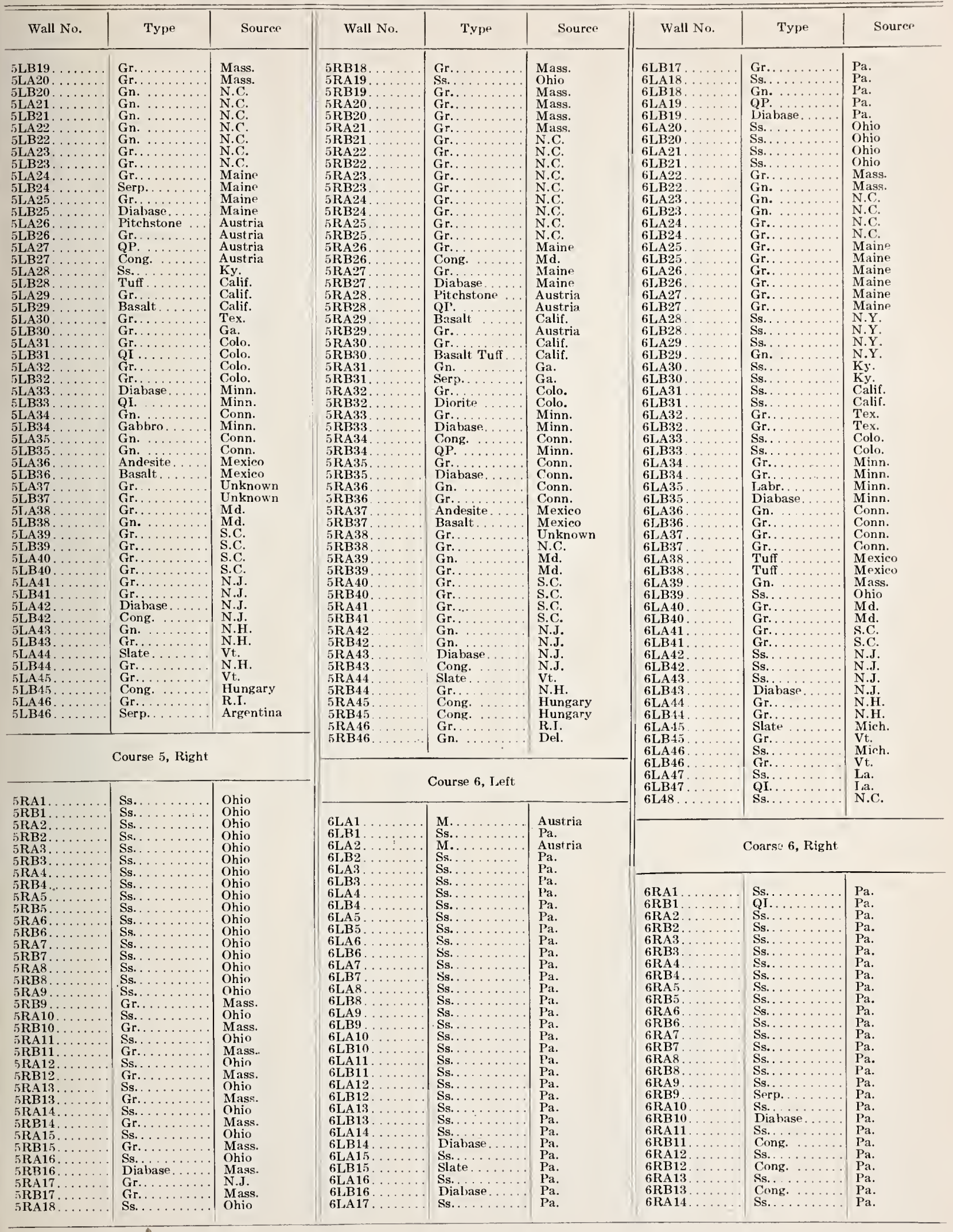


TABLE 3. Course list for idéntifying samples, by position, in wall-Continued

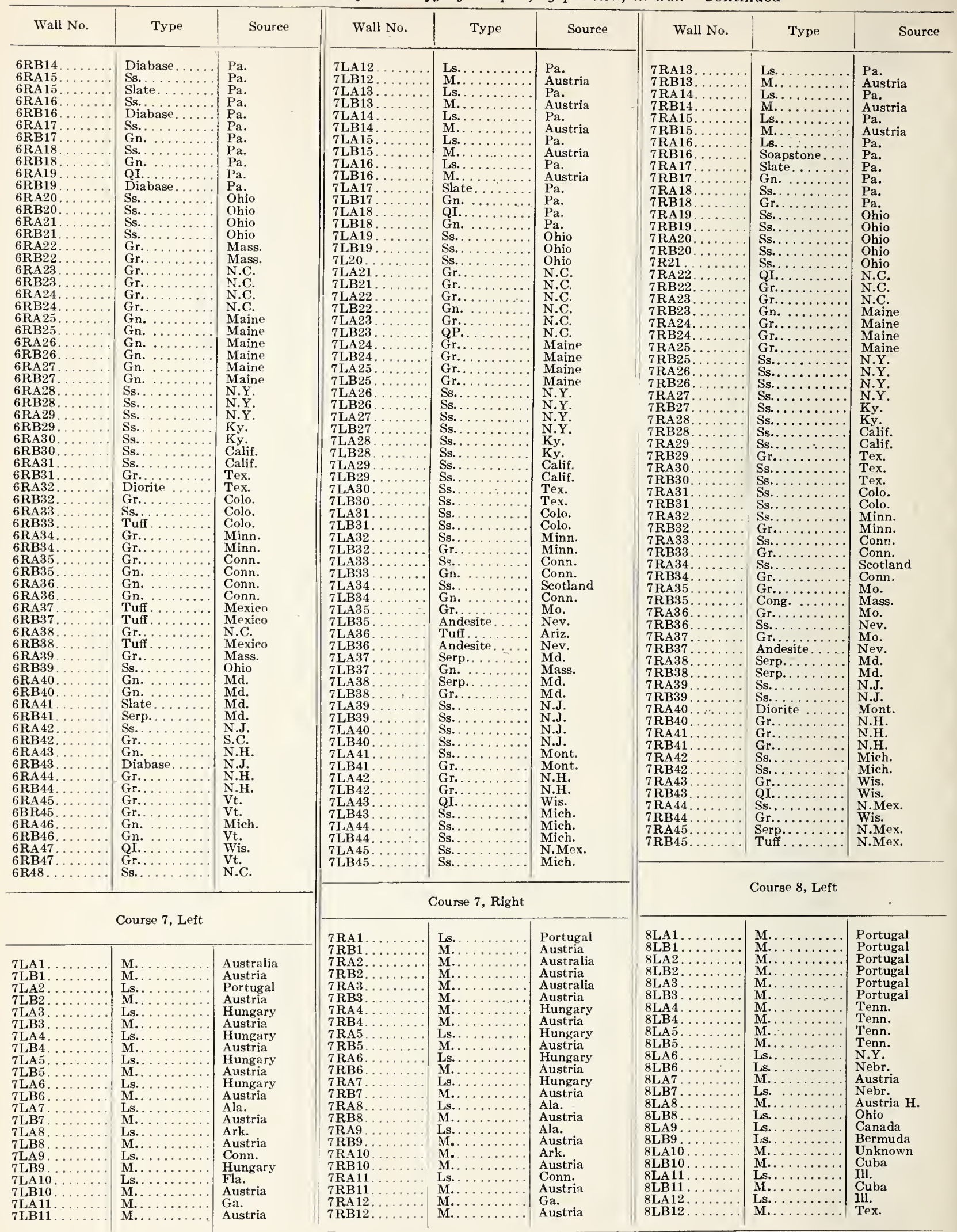


TABLE 3. Course list for identifying samples, by position, in wall-Continued

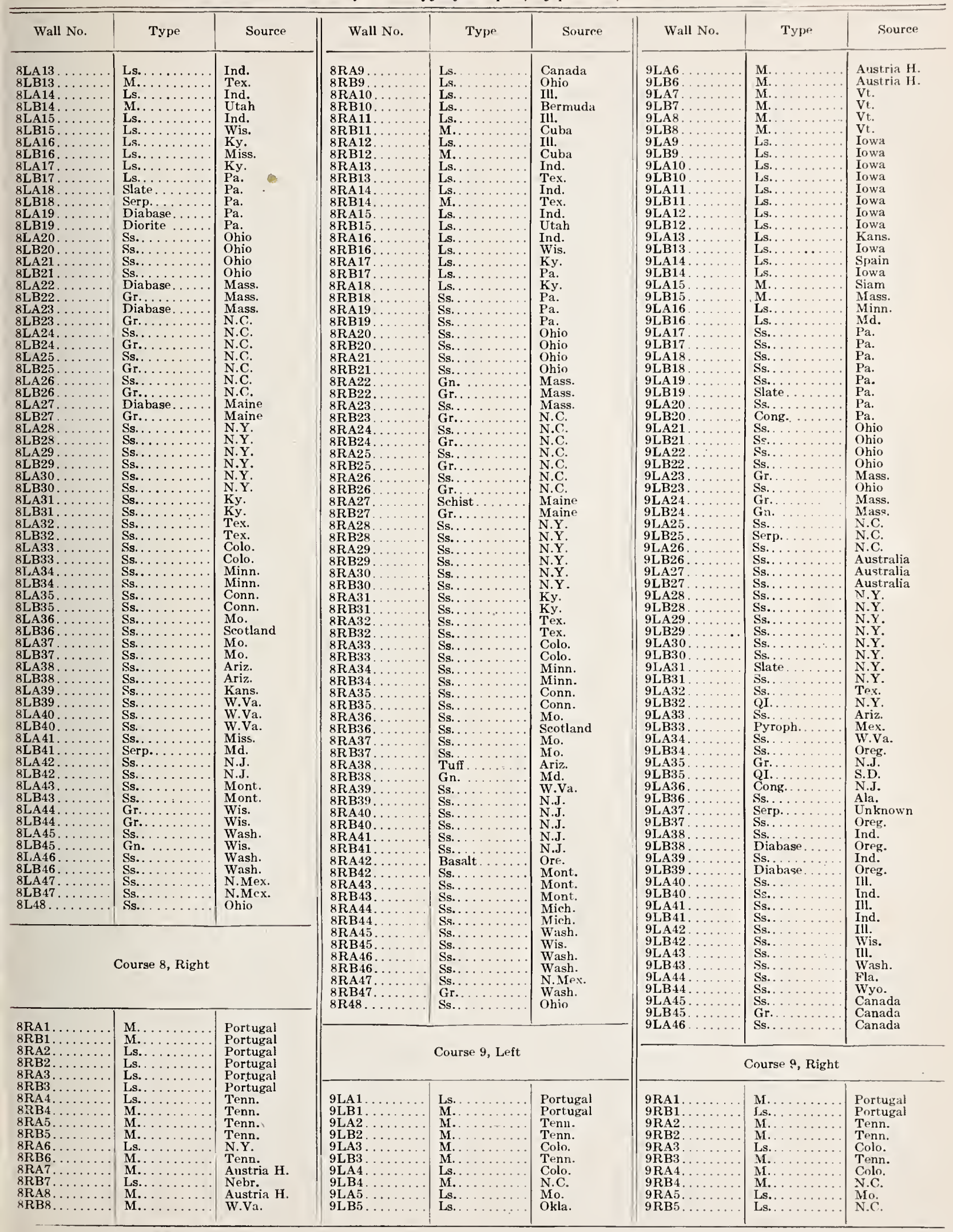


TABLE 3. Course list for identifying samples, by position, in wall-Continued

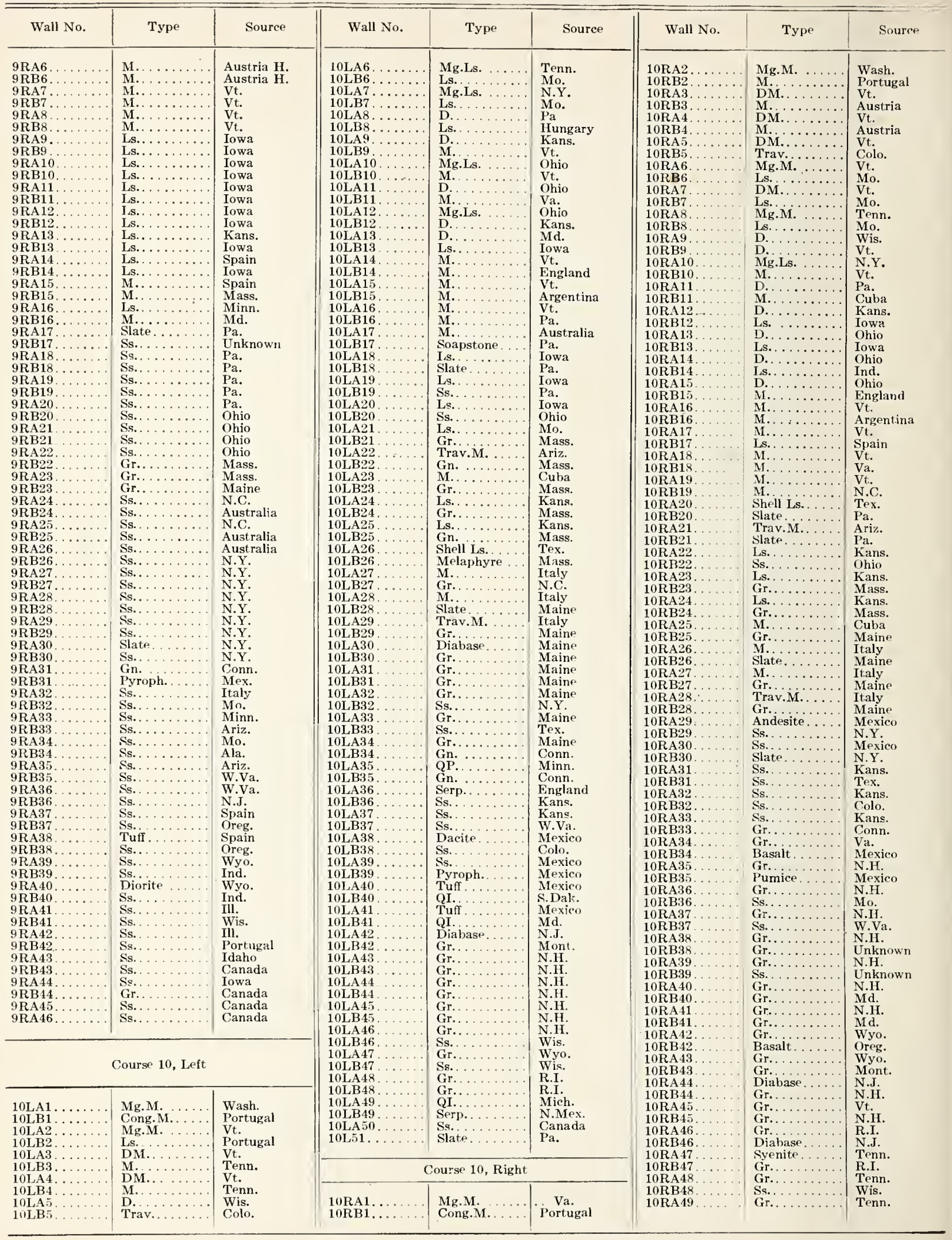


TABLE 3. Course list for identifying samples, by position, in wall-Continued

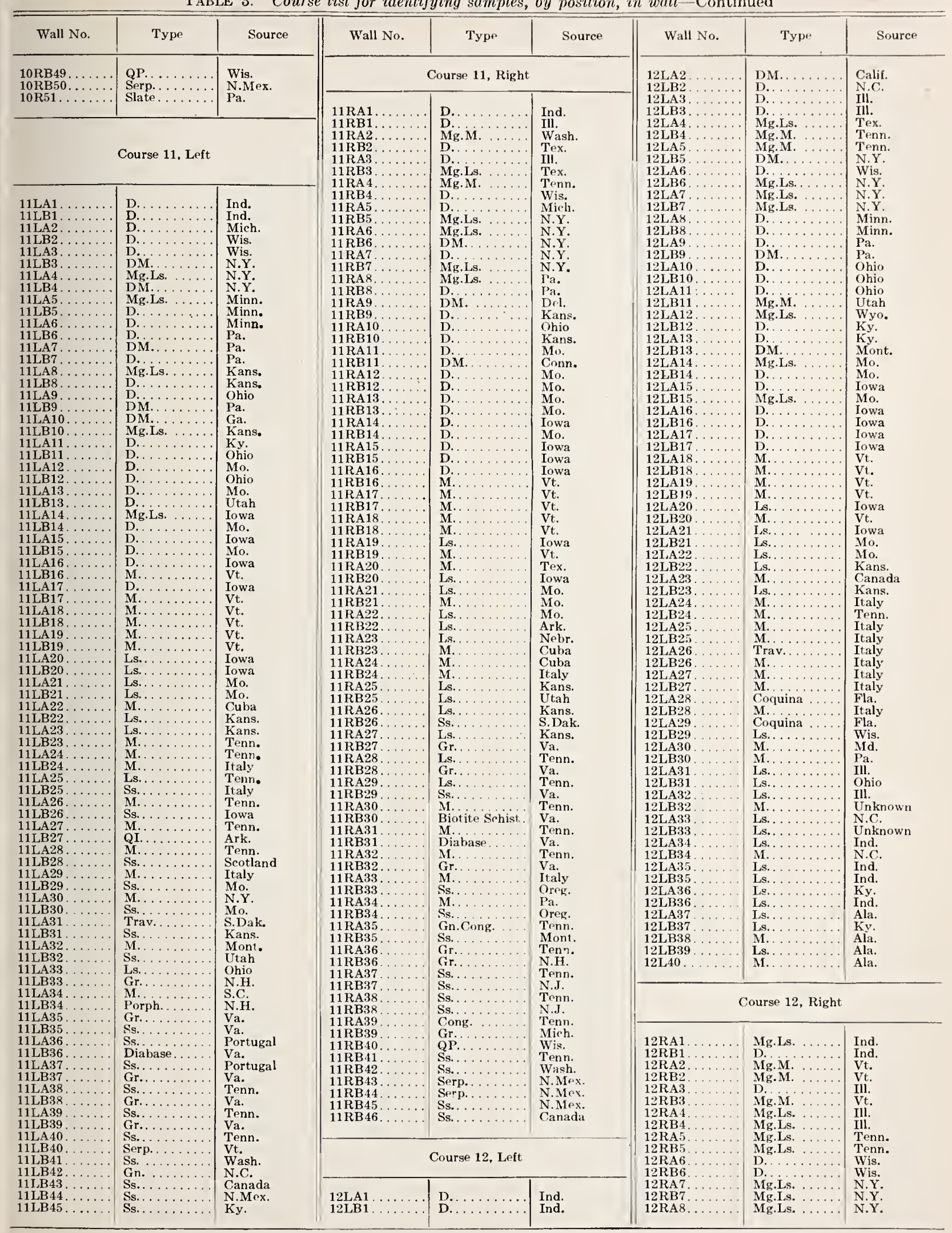


TABLE 3. Course list for identifying samples, by position, in wall-Continued

\begin{tabular}{|c|c|c|c|c|c|c|c|}
\hline Wall No. & Type & Source & Wall No. & Type & Source & Wall No. & Type \\
\hline 12RB8. & Mg.Ls. . & N.Y. & 13 LB14. & D. . & Iowa & 13RA12. & \\
\hline 12RA9. & D..... & Minn. & $13 \mathrm{LA15}$. & D. & Iowa & $13 \mathrm{RB} 12$. & D. \\
\hline 12RB9. & D......... & Minn. & $13 \mathrm{LB} 15$. & D.......... & Iowa & 13RA13. & D. \\
\hline $\begin{array}{l}\text { 12RA10. } \\
\text { 12RB10. }\end{array}$ & $\begin{array}{l}\text { DM. } \\
\text { DM... } \ldots \ldots\end{array}$ & $\begin{array}{l}\text { Pa. } \\
\text { Pa. }\end{array}$ & $\begin{array}{l}\text { 13LA16. } \\
\text { 13LB16. }\end{array}$ & Mg.Ls. . . . . & Iowa & $13 \mathrm{RB} 13$. & D.. \\
\hline 12RA11. & $\begin{array}{l}\text { DM.Ls. . . . . } \\
\text { Mg.L }\end{array}$ & $\begin{array}{l}\mathrm{Pa} . \\
\mathrm{Ky} .\end{array}$ & $\begin{array}{l}\text { 131.B16. } \\
13 \mathrm{LA} 17 .\end{array}$ & $\begin{array}{l}\text { Mg.Ls. } \ldots \ldots \\
\text { M. }\end{array}$ & $\begin{array}{l}\text { Iowa } \\
\text { Vt. }\end{array}$ & $\begin{array}{l}\text { 13RA14. } \\
\text { 13RB14. }\end{array}$ & $\mathrm{D}_{\alpha} \ldots$ \\
\hline $12 \mathrm{RB} 11$. & Mg.Ls. $\ldots \ldots$. & $\mathrm{Ky}$. & $13 \mathrm{LB17}$. & $M, \ldots \ldots \ldots$ & $\begin{array}{l}\text { Vt. } \\
\text { Vt. }\end{array}$ & $\begin{array}{l}\text { 13RB14.. } \\
\text { 13RA15.. }\end{array}$ & $\begin{array}{l}\text { Mg.M. } \\
\text { D...... }\end{array}$ \\
\hline $12 \mathrm{RA} 12$. & D......... & Ohio & $13 \mathrm{LA} 18$. & M. & Vt. & 13RB15. & D.. \\
\hline 12 RB12. & D.. & Ohio & $13 \mathrm{LB} 18$. & M. & Vt. & 13RA16. & D. \\
\hline 12RA13.. & D.. & Ohio & 13LA19. & Ls. & Iowa & $13 \mathrm{RB} 16$. & D.. \\
\hline 12RB13.. & D. . . . & Ohio & 13 LB19. & M. . & Vt. & 13RA17. & M. \\
\hline 12RA14.. & Mg.Ls. . . . . & Ohio & 13LA20. & Fossil & & 13RB17. & M.. \\
\hline 12RB14.. & D........... & Mo. & & Coral. & Iowa & 13RA18. & Ls. . \\
\hline 12RA15. & D........ & Mo. & $13 \mathrm{LB} 20$. & Ls.... & Iowa & 13 RB18. & M. . \\
\hline 12RB15. & D... & Iown & 13LA21 . & Ls. . & Iowa & 13RA19. & Ls. \\
\hline 12RA16. & D... & Iowa & $13 \mathrm{LB} 21$. & Ls. & Iowa & 13RB19. & M. . \\
\hline $12 \mathrm{RB} 16$. & D... & Iowa & 13LA22. & M.. & Cuba & 13RA20. & Ls. \\
\hline 12RA 17 & D.. & Iowa & $13 \mathrm{LB} 22$. & Ls.. & Mo. & 13RB20. & M.: \\
\hline 12RB17. & D... & Iowa & 13LA23. & M. & Austria $\mathrm{H}$. & 13RA21. & Lg. . \\
\hline 12RA18.. & M......... & Va. & 13LB23. & M. . & Cuba & $13 \mathrm{RB} 21$. & M. \\
\hline 12RB18. . & M......... & Vt. & 13LA24. . & M.. & A ustria $\mathrm{H}$. & 13RA22. & Ss.. \\
\hline 12RA19.. & M. . . . . . & Vt. & 13LB24 . & M.. & Canada & $13 \mathrm{RB} 22$. & M.. \\
\hline $12 \mathrm{RB} 19$. & M... & Vt. & 13LA25. . & Iss. . & Kans. & 13RA23. & Ls.. \\
\hline 12RA20. & M.. & Vt. & $13 \mathrm{LB} 25$. & M.. & Canada & $13 \mathrm{RB} 23$. & Ls. \\
\hline $12 \mathrm{RB} 20$. & M... & Vt. & $13 \mathrm{LA} 26$. & Ls. . & Tex. & 13RA24. & Ls.. \\
\hline $12 \mathrm{RA} 21$. & Ls.......... & Iowa & $13 \mathrm{I}, \mathrm{B} 26$. & Ls.. & Tex. & $13 \mathrm{RB} 24$. & Ls. \\
\hline $12 \mathrm{RB} 21$. & Ls............ & Mo. & $13 \mathrm{LA} 27$. & Ls.... & Tenn. & 13RA25. & M. \\
\hline 12RA22. . & M......... & Italy & 13LB27. & Ls.. & Tenn. & 13RB25. & Ls. . \\
\hline 12RB22. . & Ls......... & Kans. & 13LA28. . & M.. & Tenn. & 13RA26. & Ls. . \\
\hline 12RA23.. & Ls......... & Tenn. & 13LB28 . . & M. . & Tenn. & $13 \mathrm{RB} 26$. & Ls.. \\
\hline $12 \mathrm{RB} 23$. & Ls.... & Kans. & 13LA29... & M.. & Austria & 13RA27. & Ls.. \\
\hline 12RA24. & M... & Italy & $13 \mathrm{LB} 29 \ldots$ & Ls.. & Italy & $13 \mathrm{RB} 27$. & \\
\hline $12 \mathrm{RB} 24$ & Ls... & Tenn. & $13 \mathrm{LA} 30$. & M.. & Austria & $13 \mathrm{RA} 28$. & M.. \\
\hline 12RA25. & Trav.... & Italy & $13 \mathrm{LB} 30$. & M.. & Austria & 13RB28. & M. . \\
\hline 12RB25. & M. . . . . . & Tenn. & 13LA31. . & M.... & Austria & 13RA29. & M. \\
\hline 12RA26. & M... . & Italy & 13LB31. & M.. & Austria & 13 RB29. & M.. \\
\hline 12RB26. & M. . . . & Italy & 13LA32. & M... & Spain & 13RA30. & M.. \\
\hline 12RA27. & Coquina & Fla. & 13LB32. & Ls... & Minn. & $13 \mathrm{RB} 30$. & M.. \\
\hline $12 \mathrm{RB} 27$. & M..... & Italy & 13LA33. & M.. & Spain & 13RA31. & M.. \\
\hline 12RA28. & Coqlina. & Fla. & $13 \mathrm{LB} 33$. & M. & Mass. & 13RB31. & DM. \\
\hline 12 RB2 8 . & M...... & Italy & $13 \mathrm{LA} 34$. & M.. & Spain & 13RA32. & M. . \\
\hline 12RA29. . & M. . . . . & Ohio & $13 \mathrm{LB} 34$. & M. . & Spain & $13 \mathrm{RB} 32$. & M. : \\
\hline 12RB29. . & M..... & Italy & 13LA35. & Ls.. & Spain & 13RA33. & M.. \\
\hline 12RA30. . & Ls... & IIl. & 13LB35. & M. . . & Spain & 13RB33. & M. . \\
\hline 12RB30. & Is.... & N.Y. & 13LA36. & Ls... & Hungary & 13RA34. & \\
\hline 12RA31. & M. . . & Md. & 13LB36. & M......... & Utah & 13RB34.. & M.. \\
\hline 12RB31. & M. . & $\mathrm{Pa}$. & 13LA37. & Ls......... & N.Y. & 13RA35. & \\
\hline 12RA32. & Ls.. & Ind. & 13LB37. & & Ga. & 13RB35. & \\
\hline $12 \mathrm{RB} 32$. & Ls.. . & Pa. & 13LA38. & $M \ldots \ldots \ldots$ & Pa. & 13RA36. & Ls. . \\
\hline 12RA33. & Ls... & Ind. & $13 \mathrm{LB} 38$. & M (Ophiolyte). & N.Y. & 13RB36. & Ls.. \\
\hline 12RB33. & Ls... & Ohio & 13LA39. & M......... & $\mathrm{Pa}$. & 13RA37. & Ls.. \\
\hline 12RA34.. & Ls... & Ind. & 13LB39. & Ls. . . . . . . & $\mathrm{Pa}$. & 13RB37. & Ls. . \\
\hline 12RB34.. & Ls... & IIl. & 13LA40. & Ls............. & Ohio & 13RA38... & M.. \\
\hline 12RA35. & Ls... & Ind. & 13LB 40. & M........ & Md. & $13 \mathrm{RB} 38 .$. & Ls.. \\
\hline 12 RB35. & & Unknown & 13LA41. & $\mathrm{M} \ldots \ldots \ldots$ & Md. & 13RA39. & M. \\
\hline 12RA36. & & $\mathrm{Ky}$. & 13LB41. & M. . . . . . & S.C. & 13RB39. & Ls. \\
\hline 12RB36. & Ls............ & Ind. & 13LA42. & Ls. . . & Ill. & $13 R A 40$. & M.. \\
\hline 12RA37. . & M.......... & Ala. & $13 \mathrm{LB} 42$. & Ls.......... & IIl. & $13 \mathrm{RB} 40$. & Ls.. \\
\hline 12RB37. & M. . & Portugal & $13 \mathrm{LA} 43$. & Ls............. & $\mathrm{Ky}$. & 13RA41. & Ls.. \\
\hline 12RB38. & & Ky. & 13LB43. & Ls.......... & $\mathrm{Ky}$. & 13RB41. & Ls.. \\
\hline $12 \mathrm{RB} 39$. & & Ala. & 13LA44. & Ls. . . . ........ & Ky. & 13RA42. & Ls. . \\
\hline $12 \mathrm{RB} 40$. & $\mathrm{Ls} .$. & Ala. & $13 \mathrm{LB} 44 \ldots$ & Ls........... & $\mathrm{Ky}$. & $13 R B 42 \ldots \ldots$ & \\
\hline $12 \mathrm{R} 41 \ldots$ & M......... & Ala. & 13LA45 $\ldots$ & Ls........... & Ky. & 13RA $43 \ldots \ldots$ & Ls... \\
\hline & & & $\begin{array}{l}\text { 13LA46. } \\
13 \mathrm{LB} 46\end{array}$ & Ls.......... & $\begin{array}{l}\text { Ky. } \\
\text { Ala. }\end{array}$ & $13 \mathrm{RA44} \ldots \ldots$ & Ls. . . . . . . \\
\hline & Conurse 13 , Left & & 13LA47 .......... & Ls............. & Ala. & 13 RA45........ & Ls.............. \\
\hline 13LA1 & Mg.Ls. & & JOLDEt & Ls......... & & 13RA46..... & Ls.............. \\
\hline 13LB1. & Mg.M. & Va. & & & & $\begin{array}{l}\text { 13RB46 } \\
\text { 13RA47 }\end{array}$ & $\begin{array}{l}\text { Ls. . . . . . } \\
\text { Ls. }\end{array}$ \\
\hline 13LA2. & & IIl. & & Course 13, Right & & $13 R B 47 \ldots \ldots$ & Ls............ \\
\hline $\begin{array}{l}\text { 13LB2 } \ldots \\
\text { 13LA3 } \ldots\end{array}$ & Mg.M. $\ldots \ldots$ & $\begin{array}{l}\text { IIl. } \\
\text { Tenn. }\end{array}$ & & & & & \\
\hline 13LB3... & Mg.M. .... & Tenn. & 13RA1. & Mg.M. . . . & $\mathrm{Vt}$. & & Course 14, Left \\
\hline 13LA4 & & Wis. & 13RB1. & Mg.M. & Vt. & & \\
\hline 13LB4. & D... & Wis. & 13RA2. & & III. & & \\
\hline 13LA5. & D... & $\begin{array}{l}\text { Wis. } \\
\text { N.Y. }\end{array}$ & 13RB2. & & $\begin{array}{l}\text { Ill. } \\
\text { Tenn. }\end{array}$ & 14LA1. & D.. \\
\hline $13 \mathrm{LB} 5$. & $\mathrm{DM}_{.} .$. & $\begin{array}{l}\text { N.Y. } \\
\text { N.Y. }\end{array}$ & 13RA3. & Mg.M. . . . . & Tenn. & 14LB1. & D.. \\
\hline $13 \mathrm{LA} 6 .$. & Mg.Ls. . & $\begin{array}{l}\text { N.Y. } \\
\text { N.Y. }\end{array}$ & $13 \mathrm{RB} 3$. & Mg.M. . . . . & Tenn. & 14LA2. & D...... \\
\hline $13 \mathrm{LB} 6$. & D...... & $\begin{array}{l}\text { N.Y. } \\
\text { Minn. }\end{array}$ & $13 \mathrm{RA} 4$. & D...... & Wis. & $14 \mathrm{LB} 2$. & Mg.M. . \\
\hline 13LA7. . & D... & Minn. & $13 R B 4 \ldots$ & & Wis. & 14LA3. . & D..... \\
\hline 13LB7 . . . & D... & Minn. & 13RA5. & Mg.Ls. . . & N.Y. & 14LB3. . & D.. \\
\hline 13LA8 $\ldots$ & D....... & $\mathrm{Pa}$. & 13RB5. . & Mg.Ls. . . & N.Y. & 14LA4. . & D.. \\
\hline $13 \mathrm{LB} 8 \ldots$ & DM....... & $\mathrm{Pa}$. & 13RA6. . & DM... & N.Y. & $14 \mathrm{LB} 4 \ldots \ldots$ & D.. \\
\hline 13LA9. . & DM....... & Conn. & 13RB6. . & Mg.Ls. . & N.Y. & 14LA5. & D...... \\
\hline 13LB9. . & D..... & Ohio & 13RA7.. & D...... & Minn. & 14LB5. & Mg.M. . \\
\hline 13LA10. & Mg.Ls. . & Ohio & 13RB7. & D... & Mass. & 14LA6. & D... \\
\hline $13 \mathrm{LB} 10 \ldots \ldots$ & D......... & Ohio & $13 \mathrm{PA} 8$. & D. . . . & Pa. & 14LB6. & DM. . \\
\hline 13LA11 ....... & Mg.Ls. ...... & Ohis & 13RB8. & Mg.Ls. & Pa. & 14LA7. . & D... \\
\hline 13LB11. & D.......... & Ohio & 13RA9. & DM... & Conn. & 14LB7. . & DM.. \\
\hline 13LA12. & D.. & Ohio & 13RB9. & D. . . & Pa. & 14LA8 . . & D. . . . \\
\hline 13LB12. & D.. & N.J. & 13RA10. & D... & Ohio & $14 \mathrm{LB} 8$ & Mg.Ls. . \\
\hline $13 \mathrm{LA13}$. & D...... & Iowa & 13RB10. & D... & Ohio & 14LA9. & Mg.Ls. \\
\hline $13 \mathrm{LB} 13$. & Mg.Ls. . . & Iowa & 13RA11. & D... & Ohio & $14 \mathrm{LB} 9$ & D..... \\
\hline 13LA14. & D...... & Iowa & 13RB11. & D... & Ohio & $14 \mathrm{LA} 10$. & D... \\
\hline
\end{tabular}


TABLE 3. Course list for identifying samples, by position, in wall-Continued

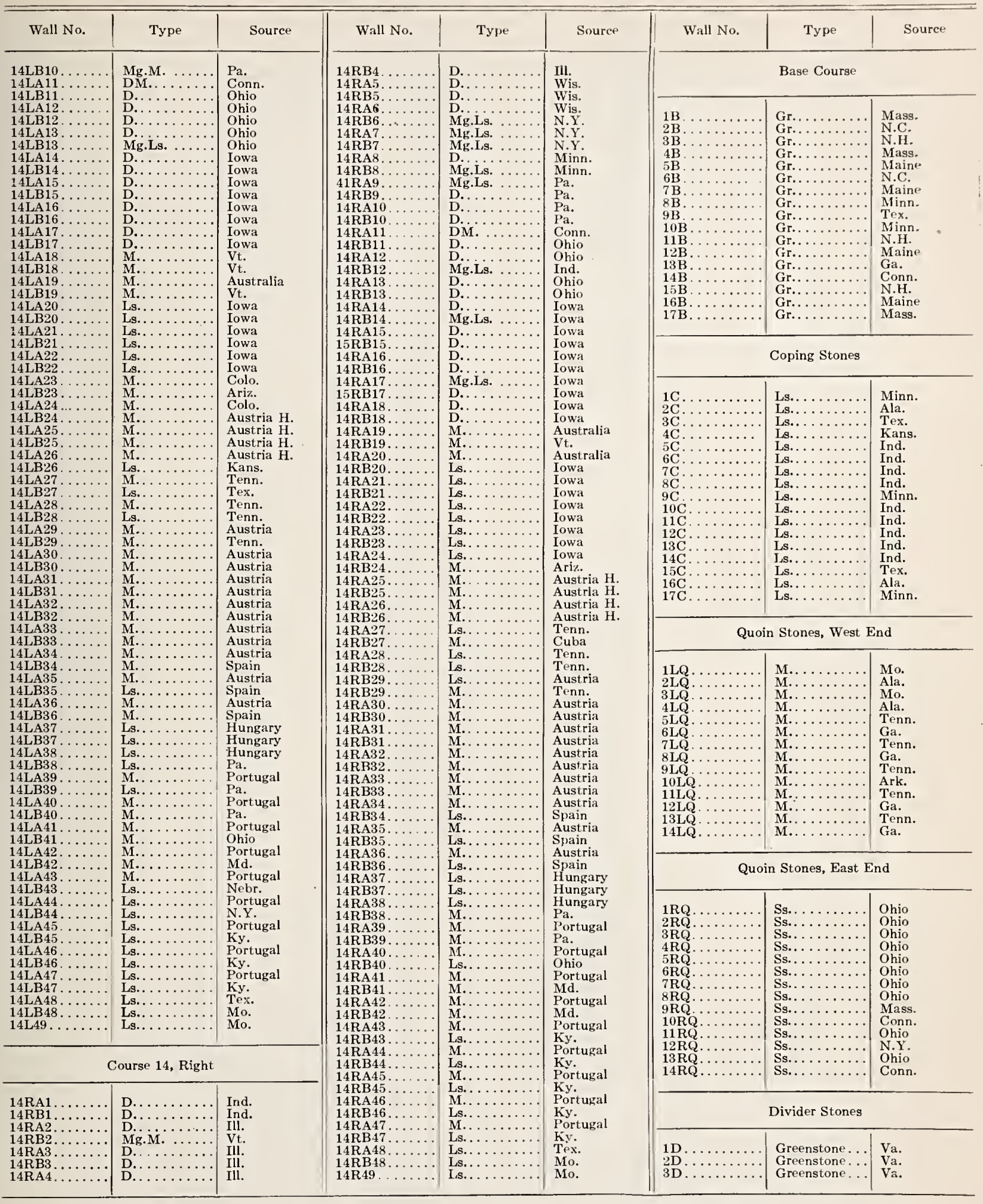




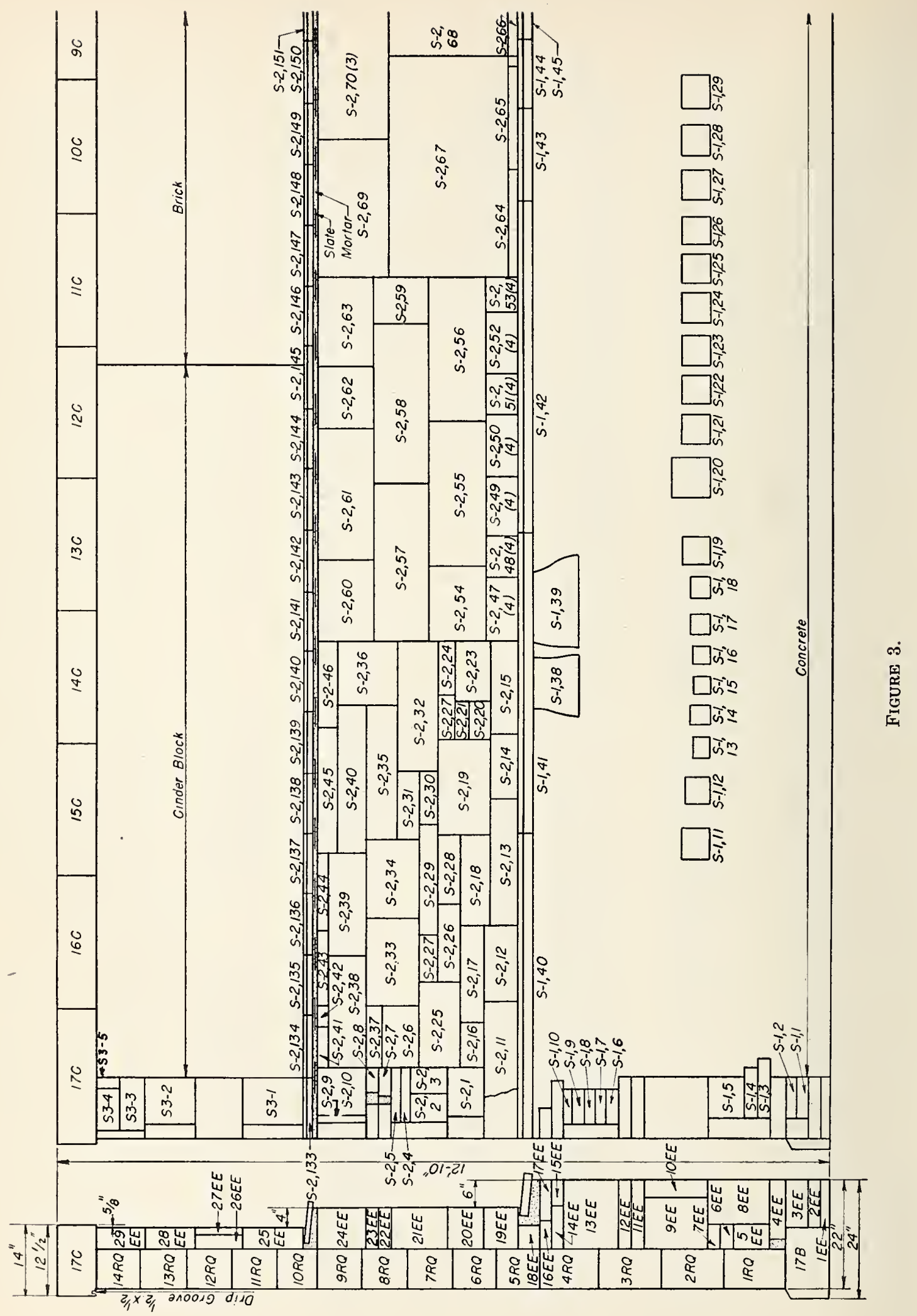




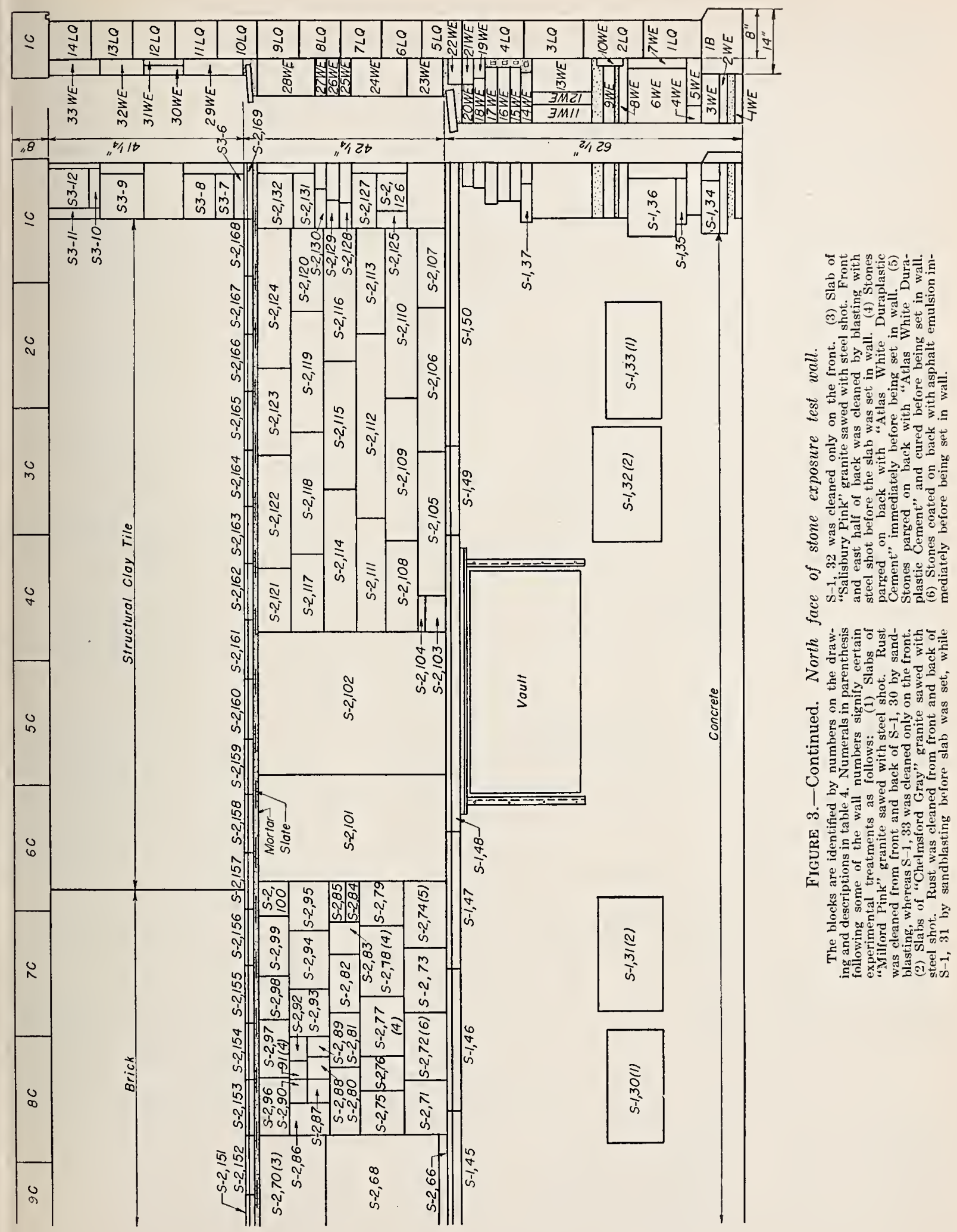


TABLE 4. Source, classification, and description of stones in back face and ends of wall

[Fixplanation of use in section 8.3. Trade names are enclosed in quotation marks, and a star $\left(^{*}\right)$ at end of line signifies that the stone has been used commereialy.]

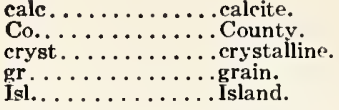

\begin{tabular}{|c|c|c|}
\hline Source & Wall No. & Description \\
\hline MeDermott, Ohio. & S-1, 1 & Gray, fine-gr, porous sandstone.* \\
\hline Do........ & $\mathrm{S}-1,2$ & Do. \\
\hline Unknown....... & $\mathrm{S}-1,3$ & Buff, fine-gr sandstone, "Golden Rod". \\
\hline Do...... & $\mathrm{S}-1,4$ & Do. \\
\hline $\begin{array}{r}\text { Do . . . . . . } \\
\text { Bedford, ind. }\end{array}$ & $\mathrm{S}-1,5$ & Do. \\
\hline Bedford, Ind...... & $S-1,6$ & Lt-buff, grade B, oolitic limestone." \\
\hline Do............. & S-1, 7 & Do. \\
\hline $\begin{array}{l}\text { Do. } \\
\text { Do. }\end{array}$ & $\begin{array}{l}S-1,8 \\
S-1,9\end{array}$ & $\begin{array}{l}\text { Gray, oolitic limestone.* } \\
\text { Do. }\end{array}$ \\
\hline Do. & $\mathrm{S}-1,10$ & Do. \\
\hline Westerly, R. I. . . & $\mathrm{S}-1,11$ & Gray, fine-gr, biotite granite.* \\
\hline Unknowi & $S-1,12$ & Bluish-gray, fine-gr biotite granite. \\
\hline Do......... & $\mathrm{S}-1,13$ & Greenish-gray, med-gr biotite granite. \\
\hline Do uincy . Mass. . . & S-1, 14 & Bluish-gray, fine-gr biotite granite. \\
\hline Quincy & $S-1,1$ & $\begin{array}{l}\text { Dark-gray, coarse-gr hornblende } \\
\text { granite. }\end{array}$ \\
\hline Maine. & S-1, & Pink, med-gr biotite granite.* \\
\hline Unknop & & Gray, med-gr biotite granite. \\
\hline Do & $\mathrm{S}-1,18$ & Dark-gray, med-gr biotite granite. \\
\hline$\cdots$ & $\mathrm{S}-1,19$ & Dark-gray, fine-gr biotite granite. \\
\hline Biel & & Gray, med-gr biotite granite. \\
\hline $\begin{array}{l}\text { Blena, Italy... } \\
\text { Quincy, Mass. }\end{array}$ & $\mathrm{S}-1,22$. & Dark-gray, coarse-gr hornblende \\
\hline & & granite.* \\
\hline Do & & Gray, med-gr hornblende granite.* \\
\hline & & $\begin{array}{l}\text { Dark-gray, coarse-gr hornblende } \\
\text { granite,* }\end{array}$ \\
\hline Baveno, & S-1 & Lt-gray, med-gr biotite granite.* \\
\hline Qu & $\mathrm{S}-1,26$ & $\begin{array}{l}\text { Dark-gray, med-gr hornblende } \\
\text { granite.* }\end{array}$ \\
\hline Italy ... & $\mathrm{S}-1,27$ & Pink, med-gr biotite granite** \\
\hline nkno & $\mathrm{S}-1,28$ & Brownish-gray, med-gr biotite granite. \\
\hline Quincy & $\mathrm{S}-1,29$ & Dark-gray, coarse-gr hornblende \\
\hline Milford, & $\mathrm{S}-1,30$. & Pink, coarse-gr biotite granite.* \\
\hline Chelmsford, & & It_oray med_or muscovite-bietite \\
\hline Mass... . . . . & $S-1,31$ & $\begin{array}{l}\text { Lt-gray, med-gr muscovite-biotite } \\
\text { granite.*}\end{array}$ \\
\hline Do & $\mathrm{S}-1,32$ & Do. \\
\hline Milford, Mass.... & $\mathrm{S}-1,33$ & Same as $S-1,30$ \\
\hline Curwensville, $\mathrm{Pa}$. & $\mathrm{S}-1,34$ & Buff, coarse-gr compact sandstone,* \\
\hline Ohio... & $\mathrm{S}-1,35 \ldots$ & Buff, coarse-gr porous sandstone.* \\
\hline $\begin{array}{l}\text { Amherst., Ohio } \\
\text { Do. }\end{array}$ & S-1,36 & $\begin{array}{l}\text { Gray, med-gr porous sandstone.* } \\
\text { Do. }\end{array}$ \\
\hline Wyoming Co., $\mathrm{Pa}$. & S-1, 38 . & $\begin{array}{l}\text { Do. } \\
\text { Dark-gray, fine-gr compact sandstone. }\end{array}$ \\
\hline Crossville, Tenn... & $S-1,39 \ldots$ & $\begin{array}{l}\text { Yellow, fine-gr, compact sandstone } \\
\text { "Crab Orchard Stone".* }\end{array}$ \\
\hline
\end{tabular}

First Water Table

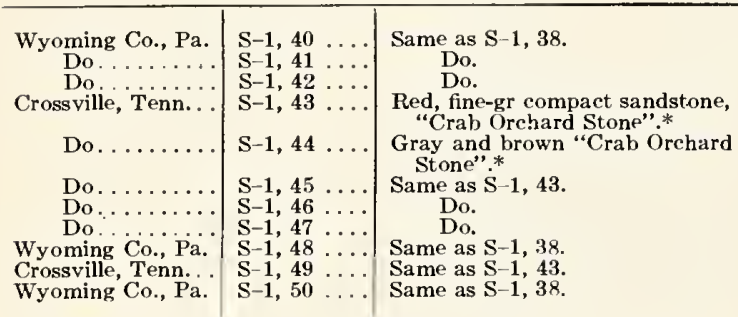

Stones in Section $2(\mathrm{~S}-2)$

\begin{tabular}{|c|c|c|}
\hline Laramie, Wyo & $\mathrm{S}-2,1$ & Lt-gray, med-gr porous sandstone.* \\
\hline Cedar Park, Tex. & $\mathrm{S}-2,2$ & Lt-gray, coarse shell-stone. \\
\hline Cedar Park, Tex. & $\mathrm{S}-\overline{2}, \overline{3}$ & Same as S-2, 2 . \\
\hline Bedford, Ind. . . . & $\mathrm{S}-2,4$ & Gray, med-gr oolitic limestone.* \\
\hline Carthage, Mo. . & $\mathrm{S}-2,5$ & Gray, med-gr cryst limestone.* \\
\hline Wilkeson, Wash. & $S-2,6$ & $\begin{array}{l}\text { Lt-bluish gray, med-gr compact } \\
\text { sandstone.* }\end{array}$ \\
\hline McDermott, Ohio. & S-2, 7 & Gray, fine-gr porous sandstone.* \\
\hline & & \\
\hline
\end{tabular}

ABBREVIATIONS

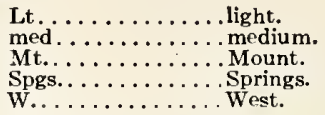

Lt........... . light.

Mt...........................

W.

\begin{tabular}{|c|c|c|}
\hline Source & Wall No. & Description \\
\hline Malone, N. Y. : . . & $\mathrm{S}-2,9$. & Pink, fine-gr compact sandstone.* \\
\hline MeDermott, Ohio. & $\mathrm{S}-2,10 \ldots$ & Buff, fine-gr porous sandstone.* \\
\hline Potomac, Md..... & $S-2,11$ to 46 & $\begin{array}{l}\text { Varicolored chlorite schist, some with } \\
\text { cleavage horizontal and some } \\
\text { vertical.* }\end{array}$ \\
\hline Barre, Vt. ....... & S-2, 47 & Gray, med-gr biotite granite** \\
\hline Concord, N. H. . . & $\mathrm{S}-2,48$ & $\begin{array}{l}\text { Gray, med-gr muscovite-biotite } \\
\text { granite.* }\end{array}$ \\
\hline Quincy, Mass... . . & $S-2,49$ & $\begin{array}{l}\text { Dark-gray, coarse-gr hornblende } \\
\text { granite.* }\end{array}$ \\
\hline $\begin{array}{l}\text { W. Chelmsford, } \\
\text { Mass. . . . . }\end{array}$ & $\mathrm{S}-2,50$ & Lt-gray, med-gr, biotite granite.* \\
\hline Frankfort, Me. & $\mathrm{S}-2,51$ & $\begin{array}{l}\text { Gray, coarse-gr biotite granite, } \\
\text { "Mt. Waldo".* }\end{array}$ \\
\hline $\begin{array}{l}\text { Sauk Rapids, } \\
\text { Minn....... }\end{array}$ & $\mathrm{S}-2,52$ & $\begin{array}{l}\text { Pinkish-gray, coarse-gr biotite } \\
\text { granite.* }\end{array}$ \\
\hline $\begin{array}{l}\text { Mountain Cross, } \\
\text { Colo. }\end{array}$ & & Bluish-oray fine-gr granodiorite * \\
\hline Mt. Airy, N. C.... & $\mathrm{S}-2,54$ to 63 & $\begin{array}{l}\text { Bluish-gray, fine-gr, granodiorite.* } \\
\text { "Mt Airy Granite" veneer.* }\end{array}$ \\
\hline Crossville, Tenn... & $\mathrm{S}-2,64 \ldots$. & $\begin{array}{l}\text { Pink, fine-gr compact sandstone, } \\
\text { "Crab Orchard Stone".* }\end{array}$ \\
\hline Do......... & $\begin{array}{l}S-2,65 \\
S-2,66\end{array}$ & $\begin{array}{l}\text { Do. } \\
\text { Do. }\end{array}$ \\
\hline Salisbury, i & $S-2,67$ & Pale pink, med-gr biotite granite.* \\
\hline & S-2, 68 & Yellowish-pink, med-gr biotite granite.* \\
\hline & S-2, 69 & Pink, coarse-gr "Opaline Granite".* \\
\hline W. Chelmsford, & $\mathrm{S}-2,70$ & Same as S-2, 67 \\
\hline Mass. . . . . . & $\mathrm{S}-2,71 \ldots$ & $\begin{array}{c}\text { Lt-gray, med-gr muscovite-biotite } \\
\text { granite.* }\end{array}$ \\
\hline Mt. Airy, N. C. & $S-2,72$ & Lt-gray, med-gr biotite granite.* \\
\hline Do. & $\mathrm{S}-2,73$ & Do. \\
\hline Do . . . . . & $\mathrm{S}-2,74$ & Do. \\
\hline $\begin{array}{l}\text { Helsinki, Finland. . } \\
\text { Concord, N. H. . . }\end{array}$ & $\begin{array}{l}\mathrm{S}-2,75 \\
\mathrm{~S}-2,76\end{array}$ & $\begin{array}{l}\text { Red, black-spotted hornblene granite.* } \\
\text { Gray, med-gr muscovite-biotite }\end{array}$ \\
\hline & $\mathrm{S}-2,77$ & $\begin{array}{l}\text { granite.* } \\
\text { Do. }\end{array}$ \\
\hline Mt. Airy, N. $\ddot{C}$. & $\mathrm{S}-2,78$ & Same as S-2, 72 . \\
\hline Milford, Mass. & $\mathrm{S}-2,79$ & Pink, coarse-gr biotite granite.* \\
\hline Concord, N. H. & $S-2,80$ & Same as $S-2,76$. \\
\hline Quincy, Mass.. & $\mathrm{S}-2,81$ & $\begin{array}{l}\text { Dark-gray, coarse-gr hornblende } \\
\text { granite.* }\end{array}$ \\
\hline Ashland & $\mathrm{S}-2,82$ & Black, med-gr olivine norite.* \\
\hline Filberton, Ga. & $\mathrm{S}-\overline{2}, 8 \overline{3}$ & Bluish-gray, fine-gr biotite granite.* \\
\hline $\begin{array}{l}\text { Bedford, Ind...... } \\
\text { Do . . . . . }\end{array}$ & $\begin{array}{l}\text { S- } 2,84 \\
\text { S-2, } 85 .\end{array}$ & $\begin{array}{l}\text { Buff, med-gr oolitic limestone.* } \\
\text { Do. }\end{array}$ \\
\hline Ortonville, Minn. . & $\mathrm{S}-2,86$ & $\begin{array}{l}\text { Pink, med-gr biotite granite, "Cold } \\
\text { Springs Agate".* }\end{array}$ \\
\hline Llano, Tex. & $\mathrm{S}-2,87$ & $\begin{array}{l}\text { Pink, brown and purple opaline } \\
\text { granite.* }\end{array}$ \\
\hline Deer Island, Me... & $\mathrm{S}-2,88$ & Pin kish-gray, coarse-gr biotite granite.* \\
\hline Mass. . . . . . . . & S-2, 89 & Bluish-gray, fine-gr biotite granite.* \\
\hline Deer Island, Me... & S $-2,90$ & $\begin{array}{l}\text { Same as } \mathrm{S}-2,88 \text {. } \\
\text { Do. }\end{array}$ \\
\hline High Pine, Me.... & $\stackrel{S}{\mathrm{~S}-2,92}$ & $\begin{array}{l}\text { Pink, coarse-gr biotite granite } \\
\text { "Swensons Pink".* }\end{array}$ \\
\hline $\begin{array}{l}\text { Derby, Vt....... } \\
\text { North Jay, Me... }\end{array}$ & $\begin{array}{l}S-2,93 \ldots \\
S-2,94 \ldots\end{array}$ & $\begin{array}{l}\text { Gray, med-gr biotite granite.* } \\
\text { Light-gray, fine-gr biotite granite.* }\end{array}$ \\
\hline Mt. Airy, N. C.... & $\mathrm{S}-2,95$ to 99 & Same as $\mathrm{S}-2,72$ in $1-$ to 3 -in. thickness.* \\
\hline Colo................ & $S-2,100 \ldots$ & Bluish-gray, fine-gr granodiorite.* \\
\hline Crossville, Tenn... & $\begin{array}{l}\mathrm{S}-2,101 \text { to } \\
124 \ldots\end{array}$ & $\begin{array}{l}\text { "Crab Orchard Stone", variegated } \\
\text { colors, some on bed and some on } \\
\text { edge, in } 3-\text { to } 6 \text { in. thicknesses.* }\end{array}$ \\
\hline Cottonwood, Kang. & $\mathrm{S}-2,125$ & Gray, coarse-gr cryst limestone.* \\
\hline Laramie, Wyo. ... & $\mathrm{S}-2,126$ & Dark-pink, med-gr porous sandstone** \\
\hline Cedar Park, Tex. . & $\mathrm{S}-2,127$ & Lt-gray, coarse shell stone.* \\
\hline Bedford, Ind...... & $\mathrm{S}-2,128$ & Gray, med-gr oolitic limestone.* \\
\hline $\begin{array}{l}\text { New Port Richey, } \\
\text { Fla. . . . . . . . }\end{array}$ & $\mathrm{S}-2,129$ & Ist-buff, fine-gr compact limestone.* \\
\hline Bedford, Ind. & $S-2,130$ & Gray, med-gr oolitic limestone.* \\
\hline & $\mathrm{S}-2,131$ & Dark-gray,fine-gr compact bluestone.* \\
\hline Boise, Idaho...... & $\mathrm{S}-2,132$ & Buff, coarse-gr porous sandstone.* \\
\hline
\end{tabular}

Marbles in second Water table

\begin{tabular}{r|c|c} 
Gardner, Mont... & S-2, 133 $\ldots$ & Gray travertine.* \\
Do........ & S-2, 134 ... & Buff travertine.*
\end{tabular}


TABLE 4. Source, classification, and description of stones in back face and ends of wall-Continued

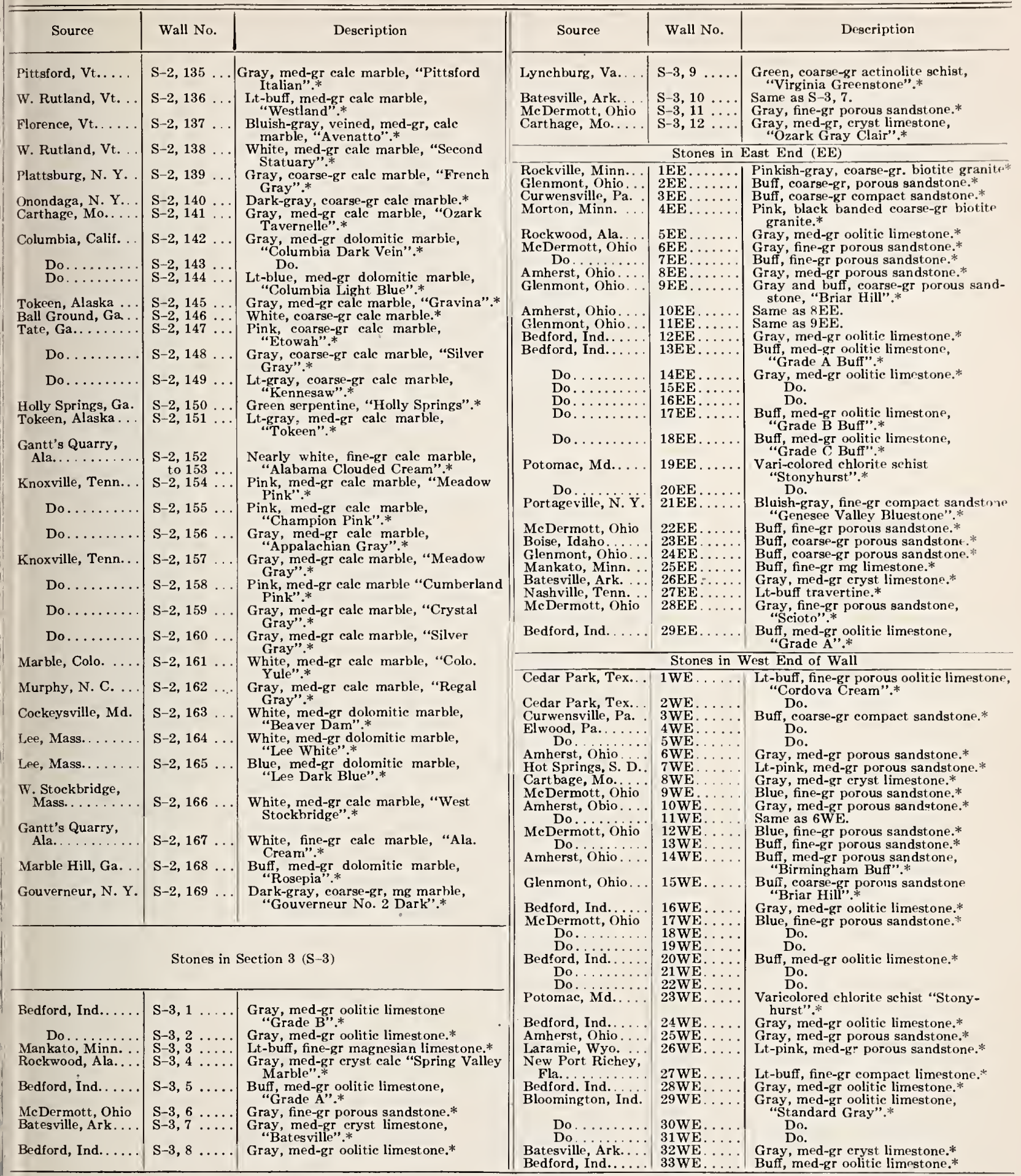







\title{
BUILDING MATERIALS AND STRUCTURES REPORTS
}

\author{
[Continued from cover page II]
}

BMS32

BMS33

BMS34

BMS35

BMS36

BMS37

BMS38

BMS39

BMS40

BMS41

BMS42

BMS43

BMS44

BMS45

BMS46

BMS47

BMS48

BMS49

BMS50

BMS551

BMS52

BMS53

BMS54

BMS55

BMS56 6

BMS57

BMS58

BMS559

BMS60

BMS61

BMS62

BMS63

BMS64

BMS65

BMS66

BMS67

BMS68

BMS69

BMS70

BMS71

BMS72

BMS73

BMS74

BMS75

BMS76

BMS77

BMS78

BMS79

BMS80

BMS81

Structural Properties of Two Brick-Concrete-Block Wall Constructions and a ConcreteBlock Wall Construction Sponsored by the National Concrete Masonry Association.. 15

Plastic Calking Materials.........................

Performance Test of Floor Coverings for Use in Low-Cost Housing: Part 1..................... 15

Stability of Sheathing Papers as Determined by Accelerated Aging.

Structural Properties of Wood-Frame Wall, Partition, Floor, and Roof Constructions With "Red Stripe" Lath Sponsored by The Weston Paper and Manufacturing Co............. $10 \&$

Structural Properties of "Palisade Homes" Constructions for Walls, Partitions, and Floors, Sponsored by Palisade Homes

Structural Properties of Two "Dunstone" Wall Constructions Sponsored by the W. E. Dunn Manufacturing Co

Structural Properties of a Wall Construction of "Pfeifer Units" Sponsored by the Wisconsin Units Co.

Structural Properties of a Wall Construction of "Knap Concrete Wall Units" Sponsored

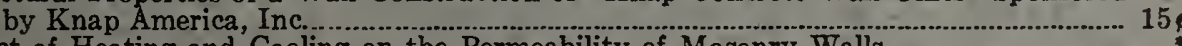

Effect of Heating and Cooling on the Permeability of Masonry Walls...................... Insulating Boards Sponsored by The Celotex Corporation............................................

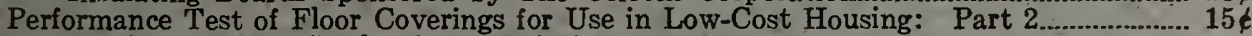

Surface Treatment of Steel Prior to Painting ……....................................................... 10

Air Infiltration Through Windows.

Structural Properties of "Scott-Bilt" Prefabricated Sheet-Steel Constructions for Walls, Floors, and Roofs Sponsored by The Globe-Wernicke Co.

Structural Properties of Prefabricated Wood-Frame Constructions for Walls, Partitions and Floors Sponsored by American Houses, Inc.

Structural Properties of "Precision-Built" Frame Wall and Partition Constructions Sponsored by the Homasote $\mathrm{C}$.

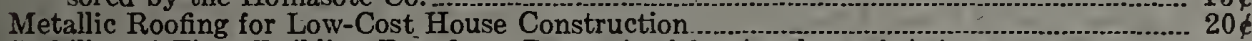

Stability of Fiber Building Boards as Determined by Accelerated Aging -.......-.................. 10

Structural Properties of "Tilecrete Type A" Floor Construction Sponsored by the Tilecrete Co.

Effect of Ceiling Insulation Upon Summer Comfort

Effect of Ceiling Insulation Upon Summer Comfort. Sponsored by the Munlock Engineering Co.

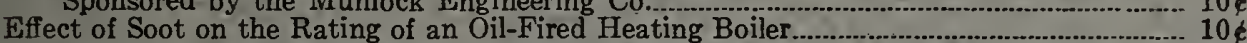

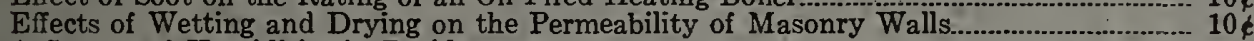

A Survey of Humidities in Residences.

Strength of Soft-Soldered Joints in Copper Tubing

Properties of Adhesives for Floor Coverings_._-

Strength, Absorption, and Resistance to Laboratory Freezing and Thawing of Building Bricks Produced in the United States.

Structural Properties of Two Nonreinforced Monolithic Concrete Wall Constructions.--.- $10 k$

Structural Properties of a Precast Joist Concrete Floor Construction. Sponsored by the Portland Cement Association.

Moisture Condensation in Building Walls

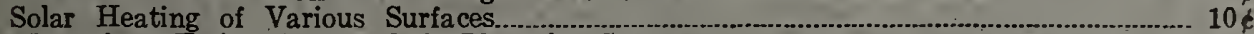

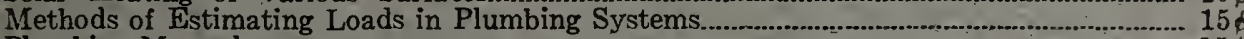

Plumbing Manual . - .

Structural Properties of "Mu-Steel" Prefabricated Sheet-Steel Constructions for Walls,

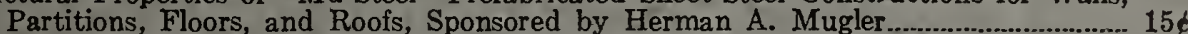

Performance Test for Floor Coverings for Use in Low-Cost Housing: Part 3..................... 20

Stability of Fiber Sheathing Boards as Determined by Accelerated Aging.............................. 10

Asphalt-Prepared Roll Roofings and Shingles

Fire Tests of Wood- and Metal-Framed Partitions

Structural Properties of "Precision-Built, Jr." Prefabricated Wood-Frame Wall Construction Sponsored by the Homasote Co..-

Indentation Characteristics of Floor Coverings.............. 10

Structural and Heat-Transfer Properties of "U. S. S. Panelbilt" Prefabricated Sheet-Steel Constructions for Walls, Partitions, and Roofs Sponsored by the Tennessee Coal, Iron \& Railroad Co.

Survey of Roofing Materials in the North Central States.

$20 k$

Effect of Outdor Exposure on the Water Permeability of Mason-.........-...- 15

Properties and Performance of Fiber Tile Boards.....

Structural, Heat-Transfer, and Water-Permeability Properties of Five Earth-Wall

Water-Distributing Systems for Buildings

$25 k$

Water-Distributing Systems for Buildsngs

Field Inspectors' Check List for Building Constructions (cloth cover, 5 x $7 \frac{1}{2}$ inches)......... $30 k$

*Out of print.

[List continued on cover page Iv] 


\section{BUILDING MATERIALS AND STRUCTURES REPORTS}

[Continued from cover page iil]

BMS82

BMS83

BMS84

BMS85

BMS86

BMS87

BMS88

BMS89

BMS90

BMS91

BMS92

BMS93

BMS94

BMS95

BMS96

BMS97

BMS98

BMS99

BMS100

BMS101

BMS102

BMS103

BMS104

BMS105

BMS106

BMS107

BMS108

BMS109

BMS110

BMS111

BMS112

BMS113

BMS114

BMS115

BMS116

BMS117

BMS118

BMS119

BMS120

BMS121

BMS122

BMS123

BMS124

BMS125
Water Permeability of Walls Built of Masonry Units

Strength of Sleeve Joints in Copper Tubing Made With Various Lead-

Dimensional Changes of Floor Coverings With Changes in Relative Humidity and Temperature.

Structural, Heat-Transfer, and Water-Permeability Properties of "Speedbrik" Wall Construction Sponsored by the General Shale Products Corporation.

A Method for Developing Specifications for Building Construction-Report of Subcommittee on Specifications of the Central Housing Committee on Research, Design, and Construction.

Recommended Building Code Requirements for New Dwelling Construction With Special Reference to War Housing.

Structural Properties of "Precision-Built, Jr." (Second Construction). Prefabricated Wood-Frame Wall Construction Sponsored by the Homasote Co..

Structural Properties of "PHC" Prefabricated Wood-Frame Constructions for Walls, Floors, and Roofs, Sponsored by the PHC Housing Corporation.

A Glossary of Housing Terms.

Fire-Resistance Classifications of Building Constructions.

Accumulation of Moisture in Walls of Frame Construction During Winter Exposure....

Water Permeability and Weathering Resistance of Stucco-Faced, Gunite-Faced, and "Knap Concrete-Unit" Walls

Tests of Cement-Water Paints and Other Waterproofings for Unit-Masonry Walls.......

Properties of a Porous Concrete of Cement and Uniform-Sized Gravel

Experimental Dry-Wall Construction With Fiber Insulating Board.

Physical Properties of Terrazzo Aggregates

Structural and Heat-Transfer Properties of "Multiple Box-Girder Plywood Panels" for Walls, Floors, and Roofs

Relative Slipperiness of Floor and Deck Surfaces

Strength and Resistance to Corrosion of Ties for Cavity Walls

Painting Steel.

Measurements of Heat Losses From Slab Floors.

Structural Properties of Prefabricated Plywood Lightweight Constructions for Walls, Partitions, Floors, and Roofs Sponsored by the Douglas Fir Plywood Association...

Paint Manual with particular reference to Federal Specifications

Laboratory Observations of Condensation in Wall Specimens.

Building Code Requirements for New Dwelling Construction.

Temperature Distribution in a Test Bungalow With Various Heating Devices....................... 10 Strength of Houses: Application of Engineering Principles to Structural Design............. 1.50

Paints for Exterior Masonry Walls

Performance of a Coal-Fired Boiler Converted to Oil

Properties of Some Lightweight-Aggregate Concretes With and Without an Air-entraining Admixture.

Fire Resistance of Structural Clay Tile Partitions.

Temperature in a Test Bungalow With Some Radiant and Jacketed Space Heaters.

A Study of a Baseboard Convector Heating System in a Test Bungalow.

Preparation and Revision of Building Codes.

Fire Resistance of Walls of Lightweight Aggregate Concrete Masonry Units

Stack Venting of Plumbing Fixtures.

Wet Venting of Plumbing Fixtures..

Fire Resistance of Walls of Gravel-Aggregate Concrete Masonry Units

Investigation of Failures of White-Coat Plasters.

Physical Properties of Some Samples of Asbestos-Cement Siding

Fire Tests of Wood-Framed Walls and Partitions with Asbestos-Cement Facings.

Fire Tests of Steel Columns Protected With Siliceous Aggregate Concrete.

Stone Exposure Test Wall.

Out of print. 